\title{
Pseudo-rotações irracionais do anel fechado
}

\section{Francisco Javier Tipán Salazar}

\author{
DISSERTAÇÃO APRESENTADA \\ $\mathrm{AO}$ \\ INSTITUTO DE MATEMÁTICA E ESTATÍSTICA \\ DA \\ UNIVERSIDADE DE SÃO PAULO \\ PARA \\ OBTENÇÃO DO TÍTULO \\ DE \\ MESTRE EM CIÊNCIAS
}

Programa: Matemática Aplicada

Orientador: Prof. Dr. Salvador Addas Zanata

Durante o desenvolvimento deste trabalho o autor recebeu auxílio financeiro da $\mathrm{CNPq}$

São Paulo, setembro de 2008 


\title{
Pseudo-rotações irracionais do anel fechado
}

\author{
Este exemplar corresponde à redação \\ final da dissertação devidamente corrigida \\ e defendida por Francisco Javier Tipán Salazar \\ e aprovada pela Comissão Julgadora.
}

Banca Examinadora:

- Prof. Dr. Salvador Addas Zanata - IME-USP.

- Prof. Dr. Fabio Armando Tal - IME-USP.

- Prof. Dr. Fernando Figueiredo de Oliveira Filho - ICEx-UFMG. 
Em memória da minha mãe. 


\section{Agradecimentos}

Em primeiro lugar, quero agradecer a infinita ajuda de meu orientador, a quem considero um grande amigo: Obrigado pelo excelente trabalho e suas sugestões. Da mesma forma, é impossível esquecer do apoio incondicional da minha família, e para todos eles, um agradecimento especial: Me sinto muito orgulhoso por vocês. Similarmente, tenho que agradecer a todos meus amigos do IME, brasileiros e estrangeiros, e a todas as "muchachas". Na verdade, é grande o número de pessoas que estiveram por trás deste projeto. Além da formação matemática que tive no IME, as lições de amizade foram meu melhor aprendizado. Por isso, para todos eles: Muito obrigado e "muchas gracias". Ainda, desejo muito agradecer aos professores que foram parte da minha banca, pois embora o tempo para as correções fosse curto, eles não tiveram problema e aceitaram o desafio. Daí que, me sinto em dívida com eles.

Finalmente, quero agradecer o apoio financero da CNPq, o qual foi fundamental para o desenvolvimento deste trabalho. 


\section{Resumo}

O conceito de número de rotação originalmente definido para homeomorfismos do círculo $\mathbb{S}^{1}$ que preservam orientação pode ser generalizado para todo homeomorfismo $h$ do anel fechado $\mathbb{S}^{1} \times[0,1]$ isotópico à identidade, onde obtemos o chamado conjunto de rotação. Neste trabalho estudamos o caso em que o conjunto de rotação de $h$ se reduz somente a um número irracional $\alpha$ (neste caso dizemos que $h$ é uma pseudo-rotação irracional), obtendo que para qualquer inteiro positivo $n$, existe um arco simples $\gamma$ que une uma componente do bordo do anel à outra, de tal modo que $\gamma$ é disjunto de seus $n$ primeiros iterados por $h$. Este resultado é um análogo do Teorema de Kwapisz concernente a difeomorfismos do toro bidimensional [14]. Posteriormente e utilizando o primeiro resultado, provamos que a rotação rígida de ângulo $\alpha$ pode ser aproximada por um homeomorfismo conjugado a $h$. Finalmente, mostramos que ser uma pseudo-rotação irracional é uma propriedade necessária para que um homeomorfismo tenha a propriedade de interseção de curvas e não tenha pontos periódicos.

Este trabalho se baseia nos resultados obtidos por Béguin, Crovisier, Le Roux e Patou [1].

Palavras-chave: número de rotação, anel, isotópico à identidade, conjunto de rotação, pseudo-rotação irracional, Kwapisz. 


\section{Abstract}

The concept of rotation number originally defined for orientation preserving homeomorphisms of the circle $\mathbb{S}^{1}$ can be generalized for any homeomorphism $h$ of closed annulus $\mathbb{S}^{1} \times[0,1]$ which is isotopic to the identity. In this setting we obtain the so called rotation set. In this work we study the case when the rotation set of $h$ is reduced to a single irrational number $\alpha$ (we say that $h$ is an irrational pseudo-rotation), and we prove that for any positive integer $n$, there exists a simple arc $\gamma$ joining one of the boundary components of annulus to the other, such that $\gamma$ is disjoint from its $n$ first iterates under $h$. This result is an analogue of a theorem of Kwapisz dealing with diffeomorphisms of the two-torus [14]. Subsequently and applying the first result, we prove that a rigid rotation of angle $\alpha$ can be approximated by a homeomorphism that is conjugate to $h$. Finally, we prove that to be an irrational pseudorotation is a necessary property in order that a homeomorphism has the curves intersection property and no periodic points.

This work is based in the results obtained by Béguin, Crovisier, Le Roux e Patou [1].

Keywords: rotation number, annulus, isotopic to the identity, rotation set, irrational pseudo-rotation, Kwapisz. 


\section{Sumário}

1 Introdução 1

$\begin{array}{llr}2 & \text { Preliminares } & 6\end{array}$

2.1 Noções básicas de dinâmica topológica . . . . . . . . . . . . 6

2.2 Homeomorfismos do círculo e número de rotação . . . . . . . . 8

2.3 Homeomorfismos do anel e o conjunto de rotação. . . . . . . . 10

2.4 A ordem cíclica no círculo e no anel . . . . . . . . . . . . 19

2.5 Intervalos de Farey . . . . . . . . . . . . . . . . . . . 19

3 O Teorema do 'arco de translação': Prova do Teorema 1.123

3.1 Enunciado do Teorema do 'arco de translação' . . . . . . . . . 23

3.2 Primeira prova do Teorema do 'arco de translação' . . . . . . . 26

4 Uma segunda prova do Teorema do 'arco de translação' $\quad 37$

4.1 Prova alternativa . . . . . . . . . . . . . . 38

4.2 Prova da Proposição 4.1 . . . . . . . . . . . . . . . . . 40

4.3 Prova da Proposição 4.2 . . . . . . . . . . . . . . . . . 47

5 O fecho da classe de conjugação de uma pseudo-rotação: $\begin{array}{ll}\text { Prova do Corolário 1.1 } & 57\end{array}$

5.1 Prova do Corolário $1.1 \ldots \ldots$. . . . . . . . . 57 
5.2 Preliminares: decomposição de homeomorfismos do disco . . . 58

5.3 Prova da Proposição 5.1 . . . . . . . . . . . . . . . 72

5.3.1 Primeira parte da prova .......... . . 80

5.3.2 Segunda parte da prova . . . . . . . . . . 84

5.3.3 Terceira parte da prova . . . . . . . . . . 88

6 Prova do Corolário $1.2 \quad 95$

6.1 Resultado de Bonatti-Guillou mais dois lemas técnicos . . . . 96

6.2 Prova do Corolário 1.2 . . . . . . . . . . . . . . . 103 


\section{Capítulo 1}

\section{Introdução}

O conceito de número de rotação foi introduzido por Poincaré [18] para comparar a dinâmica dos homeomorfismos do círculo que preservam orientação com a dinâmica das rotações rígidas. De fato, para todo homeomorfismo $h$ do círculo $\mathbb{S}^{1}=\mathbb{R} / \mathbb{Z}$ que preserva orientação está associado um único número $\tau(h)$, entre 0 e 1 , que de algum modo mede a velocidade média com que giram as órbitas de $h$ ao redor do círculo (ver Seção 2.2). Assim, se o homeomorfismo $h$ tem número de rotação $\alpha$, desejamos determinar se existe alguma semelhança de seu comportamento com o comportamento da rotação rígida $R_{\alpha}$. Neste caso, os resultados clássicos de Poincaré e Denjoy [7] ajudam a estabelecer de maneira clara os diferentes casos que podemos ter, os quais são apresentados nos seguintes itens:

- Se $\alpha=\frac{p}{q}$ (donde $p, q$ são primos entre si), então $h$ possui ao menos uma órbita periódica, todas as órbitas periódicas de $h$ possuem período primo $q$, e a ordem cíclica dos pontos de qualquer órbita periódica de $h$ é a mesma que a ordem cíclica de uma órbita da rotação $R_{\alpha}$.

- Se $\alpha$ é irracional, então $h$ não possui órbitas periódicas, e a ordem 
cíclica dos pontos de qualquer órbita de $h$ é a mesma que a ordem cíclica dos pontos de uma órbita da rotação $R_{\alpha}$.

- Se $\alpha$ é irracional, então $h$ é semi-conjugado à rotação $R_{\alpha}$, além disso, $h$ pertence ao fecho da classe de conjugação da rotação $R_{\alpha}$, e $R_{\alpha}$ pertence ao fecho da classe de conjugação de $h$ (i.e. $h$ pode ser conjugado a um homeomorfismo arbitrariamente próximo à rotação $R_{\alpha}$, e a rotação $R_{\alpha}$ pode ser conjugada a um homeomorfismo arbitrariamente próximo a $h)$.

- Se $\alpha$ é irracional e $h$ é um difeomorfismo $C^{2}$, então $h$ é conjugado à rotação $R_{\alpha}$.

A noção de número de rotação foi generalizada por Misiurewicz, Ziemian, e Franks com o objetivo de descrever a dinâmica dos homeomorfismos do anel fechado $\mathbb{A}:=\mathbb{S}^{1} \times[0,1]$ e do bi-toro $\mathbb{T}^{2}$ (ver e.g. [17]). Assim, dado um homeomorfismo $h$ do anel fechado $\mathbb{A}$ isotópico à identidade, podemos definir o conjunto de rotação de $h$, o qual é, de certo modo, o conjunto de todas as possíveis velocidades assintóticas de rotação das órbitas de $h$ ao redor do anel (ver Seção 2.3). Diferentemente do número de rotação, o conjunto de rotação é um intervalo fechado de $\mathbb{R}$, definido módulo $\mathbb{Z}$. Neste trabalho, estudaremos o caso em que o conjunto de rotação de $h$ se reduz a um só ponto. Em particular, chama-se pseudo-rotação irracional a todo homeomorfismo do anel fechado $\mathbb{A}$, isotópico à identidade, cujo conjunto de rotação se reduz a um número irracional $\alpha$ (dizemos que $\alpha$ é o ângulo da pseudo-rotação). Neste caso, nosso objetivo é determinar a relação que existe entre o comportamento de uma pseudo-rotação irracional de ângulo $\alpha$ com o comportamento da rotação rígida de ângulo $\alpha$. Para isto, três resultados importantes, obtidos por Béguin, Crovisier, Roux e Patou [1], serão demonstrados no presente trabalho. 
Antes de apresentar o primeiro resultado, vamos definir um arco essencial simples no anel $\mathbb{A}$ como um arco simples em $\mathbb{A}$ que une uma das componentes da fronteira de $\mathbb{A}$ com a outra. Assim, provaremos o seguinte teorema (que é uma variação do resultado de Kwapisz [14], concernente aos difeomorfismos do toro):

Teorema 1.1 (Teorema do 'arco de translação') Seja $h: \mathbb{A} \rightarrow \mathbb{A}$ uma pseudo-rotação irracional de ângulo $\alpha$. Então, para todo inteiro positivo $n$, existe um arco essencial simples $\gamma_{n}$ em $\mathbb{A}$, tal que os arcos $\gamma_{n}, \ldots, h^{n}\left(\gamma_{n}\right)$ são dois a dois disjuntos. Além disso, a ordem cíclica destes arcos é a mesma que a ordem cíclica do segmento vertical $\{\theta\} \times[0,1]$ e seus primeiros $n$ iterados pela rotação rígida de ângulo $\alpha$.

O Teorema 1.1 será uma conseqüência imediata de outro teorema mais técnico; nele vamos mudar a hipótese original e estudar o caso dos homeomorfismos do anel cujo conjunto de rotação seja um intervalo suficientemente 'pequeno'. Em particular, provaremos que se o conjunto de rotação de um homeomorfismo $h: \mathbb{A} \rightarrow \mathbb{A}$ está contido em um intervalo de Farey $] \frac{p}{q}, \frac{p^{\prime}}{q^{\prime}}[$, então a dinâmica de $h$ será similar à de qualquer rotação rígida de ângulo $\alpha \in] \frac{p}{q}, \frac{p^{\prime}}{q^{\prime}}\left[\right.$ até os primeiros $q+q^{\prime}-1$ iterados (ver Teorema 3.1).

Note que o Teorema 1.1 pode ser considerado como a versão em duas dimensões no caso dos homeomorfismos do círculo com número de rotação irracional, descrito anteriormente. Porém, o caso do anel é bem mais complicado que o caso do círculo. Por exemplo, a existência do arco $\gamma_{n}$ e da sua dinâmica descrita no Teorema 1.1 depende do inteiro $n$, o que não acontece no caso do círculo.

Vamos apresentar duas provas do Teorema 1.1, as mesmas que serão puramente bidimensionais, e envolvem basicamente a manipulação de certas operações definidas nos arcos essenciais simples. 
Como segundo resultado importante deste trabalho temos o seguinte corolário (ver Capítulo 5) do Teorema 1.1.

Corolário 1.1 Seja $h: \mathbb{A} \rightarrow \mathbb{A}$ uma pseudo-rotação irracional de ângulo $\alpha$. Então, a rotação rígida $R_{\alpha}$ de ângulo $\alpha$ pertence ao fecho (na topologia métrica) da classe de conjugação de $h$.

Em outras palavras, para qualquer pseudo-rotação irracional de ângulo $\alpha$, podemos obter uma conjugação que esteja arbitrariamente perto (na topologia métrica) de uma rotação irracional.

Similar ao caso anterior, o Corolário 1.1 será demonstrado de maneira imediata utilizando-se uma consequiência do Teorema 3.1 (ver Seção 5.1). De fato, com as mesmas hipóteses deste teorema, obteremos uma conjugação cuja distância a uma rotação irracional é limitada por um fator que depende inversamente do mínimo dos denominadores do intervalo de Farey. Com efeito, a dinâmica dos primeiros $q+q^{\prime}-1$ iterados por $h$ do arco essencial simples $\gamma$ (encontrado no Teorema 3.1) permitirá definir uma partição do anel $\mathbb{A}$ e a partir dela, vamos obter uma conjugação que coincide com a rotação rígida em todo ponto do anel, exceto possivelmente num dos elementos desta partição. Neste subconjunto, aplicaremos um fato chave relacionado com os homeomorfismos do disco (ver Seção 5.2), e assim obteremos um conjunto de homeomorfismos que servirá para definir uma nova conjugação cuja distância à rotação irracional será limitada pelo fator descrito anteriormente.

Por outro lado, as generalizações do Teorema de Poincaré-Birkhoff obtidas por Franks $[9,10]$ ou Bonatti e Guillou [11] também têm relação com o Teorema 1.1. Em particular, o resultado de Bonatti e Guillou relacionado a homeomorfismos $h$ do anel fechado $\mathbb{A}$ que são isotópicos à identidade, diz que se $h$ não possui pontos fixos, então existe um arco essencial simples em $\mathbb{A}$ que é disjunto da sua imagem por $h$, ou existe uma curva fechada simples 
homotopicamente não trivial em $\mathbb{A}$ que é disjunta da sua imagem por $h$. Desse modo, juntando este último fato com o Teorema 1.1, conseguimos provar (ver Capítulo 6) o seguinte corolário, o qual corresponde ao terceiro resultado importante deste trabalho.

Corolário 1.2 Seja h um homeomorfismo do anel $\mathbb{A}$, isotópico à identidade, e que não possui pontos periódicos. Então, pelo menos uma das seguintes propriedades é satisfeita:

(i) Existe uma curva fechada simples homotopicamente não trivial em $\mathbb{A}$ que é disjunta da sua imagem por $h$.

(ii) O homeomorfismo h é uma pseudo-rotação irracional, e, para todo inteiro positivo $n$, existe um arco essencial simples $\gamma_{n}$ em $\mathbb{A}$, tal que os $\operatorname{arcos} \gamma_{n}, h\left(\gamma_{n}\right), \ldots, h^{n}\left(\gamma_{n}\right)$ são dois a dois disjuntos.

Note que todo homeomorfismo do anel que preserva área, orientação e que não possui pontos periódicos, é uma pseudo-rotação irracional. De fato, se a primeira propriedade do Corolário 1.2 acontece, temos que o homeomorfismo não preservará área ou orientação, o que contradiz uma das nossas hipóteses. Portanto, só acontece a segunda propriedade.

Finalmente, resultados análogos aos do Teorema 1.1, Corolário 1.1 e Corolário 1.2 são obtidos por Béguin, Crovisier e Le Roux [2] no caso das pseudo-rotações irracionais do anel aberto $\left.\mathbb{S}^{1} \times\right] 0,1[$. Porém, as provas destes resultados são totalmente diferentes e mais complicadas, precisamente pela perda da compacidade. Por exemplo, a definição do conjunto de rotação já não é tão simples como no caso do anel fechado. 


\section{Capítulo 2}

\section{Preliminares}

Neste capítulo recordaremos algumas noções de dinâmica topológica e definiremos o conceito de conjunto de rotação para homeomorfismos do anel fechado $\mathbb{A}$, provando algumas de suas propriedades. Similarmente, apresentaremos as definições de ordem cíclica no círculo $\mathbb{S}^{1}$ e no anel $\mathbb{A}$ assim como dos intervalos de Farey, mostrando as propriedades elementares que estes intervalos satisfazem. Adicionalmente, serão descritas as notações utilizadas ao longo deste trabalho.

Não é objetivo deste capítulo fornecer um tratamento completo dos assuntos aqui abordados. Maiores detalhes podem ser encontrados em [12], [3] $\mathrm{e}[6]$.

\subsection{Noções básicas de dinâmica topológica}

Definição 2.1 Um sistema dinâmico topológico é um espaço topológico $X$ junto com uma aplicação contínua $f: X \rightarrow X$ ou um (semi) fluxo contínuo $f^{t}$ em $X$, ou seja, um (semi) fluxo $f^{t}(x)$ tal que a aplicação $(t, x) \mapsto f^{t}(x)$ seja contínua. No primeiro caso dizemos que o sistema é discreto e no segundo 
que é contínuo.

Nosso trabalho estará focado somente nos sistemas dinâmicos discretos. Assim, denotando por $(X, f)$ um sistema dinâmico topológico discreto, temos que para cada $n \in \mathbb{N}$, o $n$-ésimo iterado de $f$ é a composição $f^{n}=f \circ \cdots \circ f$ $(n-v e z e s)$. Definimos $f^{0}$ como a aplicação identidade, denotada $I d$. Se a aplicação $f$ é inversível, então $f^{-n}=f^{-1} \circ \cdots \circ f^{-1}(n-v e z e s)$. Desse modo, $f^{m+n}=f^{m} \circ f^{n}$ para $n, m \in \mathbb{N}$ (ou $n, m \in \mathbb{Z}$ se $f$ é inversível).

Definição 2.2 Seja $(X, f)$ um sistema dinâmico topológico. Se f é uma aplicação com inversa contínua, então f é chamado de homeomorfismo de $X$. Neste caso a órbita de um ponto $x \in X$ é o conjunto $O(x)=\left\{f^{n}(x): n \in \mathbb{Z}\right\}$.

Definição 2.3 Sejam $(X, f)$ e $(Y, g)$ dois sistemas dinâmicos topológicos. Os espaços $X$ e $Y$ são homeomorfos se existe uma aplicação $\psi: X \rightarrow Y$ contínua, bijetora com inversa contínua. Ainda, uma semi-conjugação de $g$ a $f$ é uma aplicação sobrejetora $\sigma: X \rightarrow Y$ satisfazendo $g \circ \sigma=\sigma \circ f$. Uma semi-conjugação inversível é chamada de conjugação. Nesse caso dizemos que $f$ e g são conjugados. A conjugação é uma relação de equivalência.

Definição 2.4 Um ponto $x \in X$ é periódico para $(X, f)$ se existe um $m>0$ tal que $f^{m}(x)=x$. O menor $m$ com esta propriedade é chamado de período primo de $x$. Quando $m=1$ dizemos que $x$ é um ponto fixo.

Agora, enunciaremos um resultado importante dentro da teoria de dinâmica topológica conhecido como o Lema de Brower, fato chave na demonstração do terceiro resultado deste trabalho. Para mais detalhes, consultar [4].

Seja $h: \mathbb{R}^{2} \rightarrow \mathbb{R}^{2}$ um homeomorfismo, então um arco simples $\alpha=p_{0} p_{1}$, $\operatorname{com} p_{0} \neq p_{1}$, é um arco de translação para $h$ se $h\left(p_{0}\right)=p_{1}$ e $\alpha \cap h(\alpha)=\left\{p_{1}\right\}$. 
Lema 2.1 (Brouwer) Seja $h: \mathbb{R}^{2} \rightarrow \mathbb{R}^{2}$ um homeomorfismo sem pontos fixos que preserva orientação e seja $\alpha$ um arco de translação para $h$. Então $h^{n}(\alpha) \cap \alpha=\emptyset$ para cada inteiro $n \notin\{-1,0,1\}$.

Corolário 2.1 Seja $h: \mathbb{R}^{2} \rightarrow \mathbb{R}^{2}$ um homeomorfismo sem pontos fixos que preserva orientação e seja $K \subset \mathbb{R}^{2}$ um conjunto compacto e conexo. Se $K \cap h(K)=\emptyset$, então $K \cap h^{n}(K)=\emptyset$ para todo inteiro $n \neq 0$.

Corolário 2.2 Seja $h: \mathbb{R}^{2} \rightarrow \mathbb{R}^{2}$ um homeomorfismo sem pontos fixos que preserva orientação, então para qualquer ponto $p \in \mathbb{R}^{2}$ tem-se que $\left\|h^{n}(p)\right\| \rightarrow$ $\infty$ quando $n \rightarrow \pm \infty$, ou seja, a órbita do ponto $p$ escapa de qualquer conjunto compacto em $\mathbb{R}^{2}$.

\subsection{Homeomorfismos do círculo e número de rotação}

Nesta seção apresentaremos alguns resultados sobre homeomorfismos do círculo, especificamente, no caso em que estes preservam orientação, culminando com a definição de número de rotação.

Para facilitar a notação, o círculo será identificado com o intervalo $[0,1)$.

Definição 2.5 Seja $\pi: \mathbb{R} \rightarrow \mathbb{S}^{1}$ definida por $\pi(x)=x(\bmod 1)$. Então a aplicação $\pi$ é chamada de recobrimento.

Adicionalmente, a orientação natural de $\mathbb{R}$ induz uma orientação no círculo $\mathbb{S}^{1}$. Portanto, para quaisquer $x, y \in \mathbb{S}^{1}$, dizemos que $x<_{0} y$ se percorrendose do ponto de referência 0 , na direção positiva, encontrarmos primeiro $x$ e depois o ponto $y$. 
Definição 2.6 Seja $f: \mathbb{S}^{1} \rightarrow \mathbb{S}^{1}$ um homeomorfismo. Então $f$ preserva orientação se para quaisquer $x, y \in \mathbb{S}^{1}$, tais que $x<_{0} y$, então $f(x)<_{f(0)}$ $f(y)$.

Nesse caso, temos que existe uma aplicação $\tilde{f}: \mathbb{R} \rightarrow \mathbb{R}$ contínua estritamente crescente tal que:

(i) $\widetilde{f}(x+1)=\widetilde{f}(x)+1, \forall x \in \mathbb{R}$,

(ii) $\pi \circ \tilde{f}=f \circ \pi$.

Qualquer $\tilde{f}$ como acima é chamada de levantamento de $f$.

Note-se que se $\widetilde{f}_{1}$ e $\widetilde{f}_{2}$ são levantamentos de $f$, então $\widetilde{f}_{2}=\widetilde{f}_{1}+k$ com $k \in \mathbb{Z}$. Ainda, para $n \geq 1, \widetilde{f}^{n}$ é monótona e $\widetilde{f}^{n}(x+k)=\widetilde{f}^{n}(x)+k$.

Teorema 2.1 Seja $f: \mathbb{S}^{1} \rightarrow \mathbb{S}^{1}$ um homeomorfismo que preserva orientação e $\widetilde{f}$ um levantamento de $f$. Definimos

$$
\tau(f, \widetilde{f}):=\lim _{n \rightarrow \infty} \frac{\widetilde{f}^{n}(x)-x}{n} .
$$

Então o limite existe e não depende de $x$. Ainda, se $\widetilde{f}_{1}$ e $\widetilde{f}_{2}$ são levantamentos de $f$ tais que $\widetilde{f}_{1}=\widetilde{f}_{2}+k$ com $k \in \mathbb{Z}$, então $\tau\left(f, \widetilde{f}_{1}\right)=\tau\left(f, \widetilde{f}_{2}\right)+k$.

Desse modo, o número de rotação $\tau(f)$ de um homeomorfismo $f$ do círculo que preserva orientação é:

$$
\tau(f)=\tau(f, \widetilde{f})(\bmod 1)
$$

onde $\tilde{f}$ é qualquer levantamento de $f$. 


\subsection{Homeomorfismos do anel e o conjunto de rotação.}

Similarmente ao caso do círculo, descreveremos algumas propriedades de homeomorfismos do anel fechado isotópicos à identidade, assim como conseqüências diretas da definição do conjunto de rotação, dada por Misiurewicz e Ziemian em [17].

Como na seção anterior, o círculo será identificado com o intervalo [0,1).

Denotemos por $\mathbb{A}=\mathbb{S}^{1} \times[0,1]$ o anel fechado e $\widetilde{\mathbb{A}}=\mathbb{R} \times[0,1]$ como seu recobrimento universal.

Definição 2.7 A aplicação $\tilde{\pi}: \widetilde{\mathbb{A}} \rightarrow \mathbb{A}$ definida como:

$$
\widetilde{\pi}(x, y):=(\pi(x), y)=(x(\bmod 1), y)
$$

chama-se de recobrimento.

Agora, dentro de nosso estudo, vamos chamar $T: \widetilde{\mathbb{A}} \rightarrow \widetilde{\mathbb{A}}$ à translação definida por $T:(x, y) \mapsto(x+1, y)$, por $p_{1}: \widetilde{\mathbb{A}} \rightarrow \mathbb{R}$ à projeção na primeira coordenada, e finalmente, para cada $\alpha \in \mathbb{R}, R_{\alpha}:(x, y) \mapsto(x+\alpha(\bmod 1), y)$ à rotação rígida de ângulo $\alpha$ no anel $\mathbb{A}$.

Definição 2.8 Suponhamos que $h: \mathbb{A} \rightarrow \mathbb{A}$ seja um homeomorfismo, então existe um homeomorfismo $\widetilde{h}: \widetilde{\mathbb{A}} \rightarrow \widetilde{\mathbb{A}}$ tal que:

$$
\tilde{\pi} \circ \widetilde{h}=h \circ \widetilde{\pi} .
$$

Qualquer homeomorfismo $\widetilde{h}$ que satisfaz as condições anteriores chama-se levantamento do homeomorfismo $h$.

Uma observação importante é que se $h$ é um homeomorfismo do anel $\mathbb{A} \mathrm{e}$ $\widetilde{h}$ é um levantamento de $h$, então $\widetilde{h}^{-1}$ é um levantamento de $h^{-1}$. 
Definição 2.9 Um homeomorfismo $h$ do anel fechado é isotópico à identidade se existe uma aplicação contínua $H: \mathbb{A} \times[0,1] \rightarrow \mathbb{A}$ que satisfaz:

(i) $H(x, 0)=h(x) \quad \forall x \in \mathbb{A}$,

(ii) $H(x, 1)=x \quad \forall x \in \mathbb{A}$,

(iii) Para cada $t \in[0,1]$, a aplicação $h_{t}: \mathbb{A} \rightarrow \mathbb{A}$ definida como $h_{t}(x)=$ $H(x, t)$ é um homeomorfismo.

Como conseqüência desta definição temos a seguinte proposição:

Proposição 2.1 Seja $h: \mathbb{A} \rightarrow \mathbb{A}$ um homeomorfismo isotópico à identidade. Então:

(i) $h$ preserva orientação,

(ii) $h$ preserva as componentes da fronteira, ou seja, $h\left(\mathbb{S}^{1} \times\{0\}\right)=\mathbb{S}^{1} \times\{0\}$ $e h\left(\mathbb{S}^{1} \times\{1\}\right)=\mathbb{S}^{1} \times\{1\}$.

(iii) $S e \widetilde{h}: \widetilde{\mathbb{A}} \rightarrow \widetilde{\mathbb{A}}$ é um levantamento de $h$, então $\widetilde{h}$ comuta com a translação $T$.

(iv) Para quaisquer inteiros $p, q$ temos $q u e \widetilde{h}^{p} \circ T^{-q}=T^{-q} \circ \widetilde{h}^{p}$.

(v) $\widetilde{h}=I d+\Phi$, onde $\Phi: \widetilde{\mathbb{A}} \rightarrow \widetilde{\mathbb{A}}$ é uma função periódica com respeito à primeira coordenada.

Demonstração. A prova é uma consequiência direta da Definição 2.9 .

Reciprocamente, qualquer homeomorfismo de $\widetilde{\mathbb{A}}$, isotópico à identidade e que comuta com $T$, é um levantamento de um homeomorfismo do anel $\mathbb{A}$ isotópico à identidade. Estamos prontos agora para definir o conjunto de rotação. 
Definição 2.10 Seja h um homeomorfismo do anel fechado isotópico à identidade e $\widetilde{h}$ um levantamento de $h$, então o conjunto de rotação de $\widetilde{h}$, que denotaremos por $\operatorname{Rot}(\widetilde{h})$, é dado por:

$$
\begin{aligned}
\operatorname{Rot}(\widetilde{h}):= & \left\{\omega \in \mathbb{R}: \text { existem sequiências }\left(n_{i}\right)_{i=1}^{\infty} \text { em } \mathbb{N} \text { e }\left(\widetilde{x}_{i}\right)_{i=1}^{\infty} \text { em } \widetilde{\mathbb{A}}\right. \\
& \text { tais que } \left.n_{i} \rightarrow \infty \text { e } \lim _{i \rightarrow \infty} \frac{p_{1}\left(\widetilde{h}^{n_{i}}\left(\widetilde{x}_{i}\right)-\widetilde{x}_{i}\right)}{n_{i}}=\omega\right\} .
\end{aligned}
$$

Observação 2.1 O conjunto de rotação de $\widetilde{h}$ é um intervalo compacto.

Demonstração. Inicialmente, note que uma maneira mais compacta de definir $\operatorname{Rot}(\widetilde{h})$ é:

$$
\operatorname{Rot}(\widetilde{h})=\bigcap_{n \geq 1} \overline{\cup K_{k}(\widetilde{h})}
$$

onde,

$$
K_{k}(\widetilde{h}):=\left\{\frac{p_{1}\left(\widetilde{h}^{k}(\widetilde{x})-\widetilde{x}\right)}{k}: \widetilde{x} \in \widetilde{\mathbb{A}}\right\} .
$$

Pelo item (iv) da Proposição 2.1 temos que, para todo $\ell \in \mathbb{Z}$,

$$
p_{1}\left(\widetilde{h}^{k}(\widetilde{x}+(\ell, 0))-(\widetilde{x}+(\ell, 0))=p_{1}\left(\widetilde{h}^{k}(\widetilde{x})-\widetilde{x}\right) .\right.
$$

Portanto,

$$
K_{k}(\widetilde{h})=\left\{\frac{p_{1}\left(\widetilde{h}^{k}(\widetilde{x})-\widetilde{x}\right)}{k}: \widetilde{x} \in[0,1] \times[0,1]\right\} .
$$

Dado que a conexidade e a compacidade são propriedades preservadas pela continuidade, podemos ver por $(2.2)$ que $K_{k}(\widetilde{h})$ é um intervalo compacto.

Desse modo, vamos mostrar primeiramente que o conjunto $\operatorname{Rot}(\widetilde{h})$ é um intervalo. Por absurdo, suponhamos que ele não é um intervalo. Então, 
existem $x, y \in \operatorname{Rot}(\widetilde{h}), x<y$, tal que para algum $z, x<z<y$, teremos que $z \notin \operatorname{Rot}(\widetilde{h})$. Seja agora

$$
A_{n}=\overline{\bigcup_{k \geq n} K_{k}(\widetilde{h})}
$$

É fácil ver que

$$
A_{n+1} \subset A_{n}
$$

Por (2.1), vemos que $x, y \in A_{n}$ para todo $n \geq 1$. Assim, pelo absurdo, existe um $n_{0} \geq 1$ tal que $z \notin A_{n_{0}}$. Por (2.3) podemos dizer que $z \notin A_{n}$ para todo $n \geq n_{0}$. Agora, fixemos um certo $k \geq n_{0}$. Teremos então que $z \notin K_{k}(\widetilde{h})$. Denotando por $a:=\inf \left(K_{k}(\widetilde{h})\right)$, podemos supor que $z<a$. Por outro lado, seja $\omega$ qualquer elemento do conjunto $\operatorname{Rot}(\widetilde{h})$. Portanto, existem seqüências $\left(n_{i}\right)_{i=1}^{\infty}$ em $\mathbb{N}$ e $\left(\widetilde{x}_{i}\right)_{i=1}^{\infty}$ em $[0,1] \times[0,1]$ tal que $n_{i} \rightarrow \infty$ e

$$
\lim _{i \rightarrow \infty} \frac{p_{1}\left(\widetilde{h}^{n_{i}}\left(\widetilde{x}_{i}\right)-\widetilde{x}_{i}\right)}{n_{i}}=\omega .
$$

Escrevendo $n_{i}=q_{i} k+r$, onde $0 \leq r \leq k-1$, vemos que

$$
\frac{p_{1}\left(\widetilde{h}^{n_{i}}\left(\widetilde{x}_{i}\right)-\widetilde{x}_{i}\right)}{n}=\frac{p_{1}\left(\widetilde{h}^{q_{i} k+r}\left(\widetilde{x}_{i}\right)-\widetilde{x}_{i}\right)}{q_{i} k+r} .
$$

Logo,

$$
\begin{aligned}
\frac{p_{1}\left(\widetilde{h}_{i} q_{i}^{k+r}\left(\widetilde{x}_{i}\right)-\widetilde{x}_{i}\right)}{q_{i} k+r}= & \frac{p_{1}\left(\widetilde{h}^{k}\left(\widetilde{h}^{\left(q_{i}-1\right) k+r}\left(\widetilde{x}_{i}\right)\right)-\widetilde{h}^{\left(q_{i}-1\right) k+r}\left(\widetilde{x}_{i}\right)+\right.}{q_{i} k+r} \\
& \frac{\widetilde{h}^{k}\left(\widetilde{h}^{\left(q_{i}-2\right) k+r}\left(\widetilde{x}_{i}\right)\right)-\widetilde{h}^{\left(q_{i}-2\right) k+r}\left(\widetilde{x}_{i}\right)+\ldots+}{q_{i} k+r} \\
& \frac{\widetilde{h}^{k}\left(\widetilde{h}^{r}\left(\widetilde{x}_{i}\right)\right)-\widetilde{h}^{r}\left(\widetilde{x}_{i}\right)+\left(\widetilde{h}^{r}\left(\widetilde{x}_{i}\right)-\widetilde{x}_{i}\right)}{q_{i} k+r} .
\end{aligned}
$$

Daí que 


$$
\begin{aligned}
\frac{p_{1}\left(\widetilde{h}^{n}\left(\widetilde{x}_{i}\right)-\widetilde{x}_{i}\right)}{n_{i}} & \geq \frac{q_{i} \cdot a \cdot k+\left(\widetilde{h}^{r}\left(\widetilde{x}_{i}\right)-\widetilde{x}_{i}\right)}{q_{i} k+r} \\
& =\frac{a \cdot k+\frac{\left(\widetilde{h}^{r}\left(\widetilde{x}_{i}\right)-\widetilde{x}_{i}\right)}{q_{i}}}{k+\frac{r}{q_{i}}} .
\end{aligned}
$$

Pela compacidade do conjunto $K_{r}(\widetilde{h})$, a expressão $\left(\widetilde{h}^{r}\left(\widetilde{x}_{i}\right)-\widetilde{x}_{i}\right)$ vai ser limitada. Portanto, tomando o limite quando $i \rightarrow \infty$, obtemos

$$
z<a \leq \omega
$$

Dado que $\omega$ é qualquer elemento do conjunto $\operatorname{Rot}(\widetilde{h})$, tomando $\omega=x$, chegamos a um absurdo. Isto mostra que o conjunto $\operatorname{Rot}(\widetilde{h})$ é um intervalo.

Nosso seguinte passo é provar que o conjunto $\operatorname{Rot}(\widetilde{h})$ é limitado. Para isso, basta mostrar que o conjunto $A_{1}$ é limitado. Assim, seja $k$ um inteiro positivo qualquer. Para $\widetilde{x} \in[0,1] \times[0,1]$, escrevamos $\frac{p_{1}\left(\widetilde{h}^{k}(\widetilde{x})-\widetilde{x}\right)}{k}$ da forma

$$
\frac{p_{1}\left(\widetilde{h}\left(\widetilde{h}^{k-1}(\widetilde{x})\right)-\widetilde{h}^{k-1}(\widetilde{x})+\widetilde{h}\left(\widetilde{h}^{k-2}(\widetilde{x})\right)-\widetilde{h}^{k-2}(\widetilde{x})+\ldots+\widetilde{h}(\widetilde{x})-\widetilde{x}\right)}{k} .
$$

Dado que $K_{1}(\widetilde{h})$ é um intervalo compacto, então existe uma constante $L>0$ tal que $K_{1}(\widetilde{h}) \subset[-L, L]$. Por outra parte, para cada $i \in\{0, \ldots, k-1\}$, a expressão $p_{1}\left(\widetilde{h}\left(\widetilde{h}^{k-i-1}(\widetilde{x})\right)-\widetilde{h}^{k-i-1}(\widetilde{x})\right)$ pertence ao intervalo $[-L, L]$.

Portanto,

$$
-L \leq \frac{p_{1}\left(\widetilde{h}^{k}(\widetilde{x})-\widetilde{x}\right)}{k} \leq L \quad \text { para todo } \quad \widetilde{x} \in[0,1] \times[0,1]
$$

Conseqüentemente, $K_{k}(\widetilde{h}) \subset[-L, L]$ para todo $k \in \mathbb{N}$. Isto mostra que o conjunto $A_{1}$ é limitado.

Finalmente, por (2.1) segue imediatamente que $\operatorname{Rot}(\widetilde{h})$ é um conjunto fechado. Portanto, $\operatorname{Rot}(\widetilde{h})$ é um intervalo compacto. 
Observemos agora que para quaisquer inteiros $p, q$, a aplicação $\widetilde{h}^{q} \circ T^{-p}$ é um levantamento de $h^{q}$. Usando novamente o fato de que $\widetilde{h}$ e $T$ comutam, o próximo lema diz:

$$
\left(\widetilde{h}^{q} \circ T^{-p}\right)(x, y)=\widetilde{h}^{q}(x-p, y)=\widetilde{h}^{q}(x, y)-(p, 0)=\left(T^{-p} \circ \widetilde{h}^{q}\right)(x, y),
$$

para todo $(x, y) \in \mathbb{R}^{2}$.

Lema 2.2 Para quaisquer par $(p, q)$ de inteiros, o conjunto de rotação de $\widetilde{h}^{q} \circ T^{-p}$ é dado por

$$
\operatorname{Rot}\left(\widetilde{h}^{q} \circ T^{-p}\right)=q \cdot \operatorname{Rot}(\widetilde{h})-p .
$$

Demonstração. Seja $\omega \in \operatorname{Rot}\left(\widetilde{h}^{q} \circ T^{-p}\right)$, então existem seqüências $\left(n_{i}\right)_{i=1}^{\infty} \mathrm{e}$ $\left(\widetilde{x}_{i}\right)_{i=1}^{\infty}$ tais que $n_{i} \rightarrow \infty$ e

$$
\lim _{i \rightarrow \infty} \frac{p_{1}\left(\left(\widetilde{h}^{q} \circ T^{-p}\right)^{n_{i}}\left(\widetilde{x}_{i}\right)-\widetilde{x}_{i}\right)}{n_{i}}=\omega
$$

Como $\tilde{h}$ e $T$ comutam, tem-se

$$
\frac{p_{1}\left(\left(\widetilde{h}^{q} \circ T^{-p}\right)^{n_{i}}\left(\widetilde{x}_{i}\right)-\widetilde{x}_{i}\right)}{n_{i}}=\frac{p_{1}\left(\widetilde{h}^{q n_{i}}\left(\widetilde{x}_{i}\right)-\widetilde{x}_{i}\right)-n_{i} p}{n_{i}}=q\left(\frac{p_{1}\left(\widetilde{h}^{q n_{i}}\left(\widetilde{x}_{i}\right)-\widetilde{x}_{i}\right)}{q n_{i}}\right)-p .
$$

Tomando o limite quando $i \rightarrow \infty$, obtemos que

$$
\lim _{i \rightarrow \infty} \frac{p_{1}\left(\widetilde{h}^{q n_{i}}\left(\widetilde{x}_{i}\right)-\widetilde{x}_{i}\right)}{q n_{i}}=\frac{\omega+p}{q},
$$

então $\frac{\omega+p}{q}$ pertence ao conjunto de rotação de $\tilde{h}$. Logo, 


$$
\operatorname{Rot}\left(\tilde{h^{q}} \circ T^{-p}\right) \subset q \cdot \operatorname{Rot}(\tilde{h})-p .
$$

Por outro lado, dado $\omega \in \operatorname{Rot}(\widetilde{h})$, existem seqüências $\left(n_{i}\right)_{i=1}^{\infty}$ e $\left(\widetilde{x}_{i}\right)_{i=1}^{\infty}$ tal que $n_{i} \rightarrow \infty$ e

$$
\lim _{i \rightarrow \infty} \frac{p_{1}\left(\widetilde{h}^{n_{i}}\left(\widetilde{x}_{i}\right)-\widetilde{x}_{i}\right)}{n_{i}}=\omega .
$$

Anteriormente, mostramos que $K_{k}(\widetilde{h}) \subset[-L, L]$ para qualquer $k \geq 1$. Isso implica que, para todo $\widetilde{z} \in \widetilde{\mathbb{A}}$ e $0 \leq r \leq q-1$,

$$
\left|p_{1}\left(\tilde{h^{r}}(\tilde{z})-\tilde{z}\right)\right| \leq M
$$

onde $M=L(q-1)$.

Escrevendo $n_{i}=q k_{i}+r$, onde $0 \leq r \leq q-1$, temos que

$$
\begin{aligned}
\left|\frac{p_{1}\left(\widetilde{h}^{n_{i}}\left(\widetilde{x}_{i}\right)-\widetilde{x}_{i}\right)}{n_{i}}-\frac{p_{1}\left(\widetilde{h}^{q k_{i}}\left(\widetilde{x}_{i}\right)-\widetilde{x}_{i}\right)}{q k_{i}}\right| \leq & \left|\frac{p_{1}\left(\widetilde{h}^{n_{i}}\left(\widetilde{x}_{i}\right)-\widetilde{x}_{i}\right)}{n_{i}}-\frac{p_{1}\left(\widetilde{h}^{n_{i}}\left(\widetilde{x}_{i}\right)-\widetilde{x}_{i}\right)}{q k_{i}}\right|+ \\
= & \left|\frac{p_{1}\left(\widetilde{h}^{n_{i}}\left(\widetilde{x}_{i}\right)-\widetilde{x}_{i}\right)}{q k_{i}}-\frac{p_{1}\left(\widetilde{h}^{q k_{i}}\left(\widetilde{x}_{i}\right)-\widetilde{x}_{i}\right)}{\left.q k_{i}\left(\widetilde{x}_{i}\right)-\widetilde{x}_{i}\right)}\right| \cdot \frac{r}{n_{i}} \mid \\
& \mid p_{1}\left(\widetilde{h}^{r}\left(\widetilde{h}^{q k_{i}}\left(\widetilde{x}_{i}\right)\right)-\widetilde{h}^{q k_{i}}\left(\widetilde{x}_{i}\right) \mid \cdot \frac{1}{q k_{i}} .\right.
\end{aligned}
$$

Uma vez que $\frac{p_{1}\left(\widetilde{h}^{n_{i}}\left(\widetilde{x}_{i}\right)-\widetilde{x}_{i}\right)}{n_{i}} \rightarrow \omega, \frac{r}{q k_{i}} \rightarrow 0, \frac{1}{q k_{i}} \rightarrow 0$ quando $i \rightarrow \infty \mathrm{e}$ $\left|p_{1}\left(\widetilde{h}^{r}\left(\widetilde{h}^{q k_{i}}\left(\widetilde{x}_{i}\right)\right)-\widetilde{h}^{q k_{i}}\left(\widetilde{x}_{i}\right)\right)\right| \leq M$, então

$$
\lim _{i \rightarrow \infty} \frac{p_{1}\left(\left(\widetilde{h}^{q}\right)^{k_{i}}\left(\widetilde{x}_{i}\right)-\widetilde{x}_{i}\right)}{k_{i}}=q \omega,
$$

ou seja, 
$\lim _{i \rightarrow \infty} \frac{p_{1}\left(\left(\widetilde{h}^{q} \circ T^{-p}\right)^{k_{i}}\left(\widetilde{x}_{i}\right)-\widetilde{x}_{i}\right)}{k_{i}}=\lim _{i \rightarrow \infty} \frac{p_{1}\left(\left(\widetilde{h}^{q}\right)^{k_{i}}\left(\widetilde{x}_{i}\right)-\left(k_{i} p, 0\right)-\widetilde{x}_{i}\right)}{k_{i}}=q \omega-p$.

Daí podemos concluir que $q \omega-p \in \operatorname{Rot}\left(\widetilde{h}^{q} \circ T^{-p}\right)$, o que implica que

$$
q \cdot \operatorname{Rot}(\widetilde{h})-p \subset \operatorname{Rot}\left(\widetilde{h}^{q} \circ T^{-p}\right) .
$$

Assim, finalmente, $\operatorname{Rot}\left(\widetilde{h}^{q} \circ T^{-p}\right)=q \cdot \operatorname{Rot}(\widetilde{h})-p$.

Em particular, o conjunto de rotação de dois levantamentos diferem por um inteiro, e portanto, o conjunto de rotação de $h$ está bem definido como um intervalo de $\mathbb{R} / \mathbb{Z}$. Assim, como para os homeomorfismos do círculo, definimos

$$
\operatorname{Rot}(h)=\operatorname{Rot}(\widetilde{h})(\bmod 1)
$$

onde $\widetilde{h}$ é qualquer levantamento de $h$.

Proposição 2.2 O conjunto de rotação é invariante com respeito à conjugação por homeomorfismo de $\mathbb{A}$ que sejam isotópicos à identidade.

Demonstração. Sejam $f$ e $g$ homeomorfismos do anel fechado $\mathbb{A}$, isotópicos à identidade tais que $f=\sigma \circ g \circ \sigma^{-1}$, onde $\sigma$ é um homeomorfismo isotópico à identidade. Detonemos por $\widetilde{g}$ e $\widetilde{\sigma}$ levantamentos de $g$ e $\sigma$, respectivamente. Note que $\tilde{f}=\widetilde{\sigma} \circ \widetilde{g} \circ \widetilde{\sigma}^{-1}$ também é um levantamento de $f$.

Agora, seja $\omega \in \operatorname{Rot}(\widetilde{f})$. Portanto, existem seqüências $\left(n_{i}\right)_{i=1}^{\infty}$ e $\left(\widetilde{x}_{i}\right)_{i=1}^{\infty}$ tais que $n_{i} \rightarrow \infty$ e

$$
\lim _{i \rightarrow \infty} \frac{p_{1}\left(\widetilde{f}^{n_{i}}\left(\widetilde{x}_{i}\right)-\widetilde{x}_{i}\right)}{n_{i}}=\omega .
$$

Como $\widetilde{f}^{n_{i}}=\widetilde{\sigma} \circ \widetilde{g}^{n_{i}} \circ \widetilde{\sigma}^{-1}$, então 


$$
\lim _{i \rightarrow \infty} \frac{p_{1}\left(\tilde{\sigma} \circ \widetilde{g}^{n_{i}} \circ \widetilde{\sigma}^{-1}\left(\widetilde{x}_{i}\right)-\widetilde{x}_{i}\right)}{n_{i}}=\omega .
$$

A expressão do limite pode ser escrita como

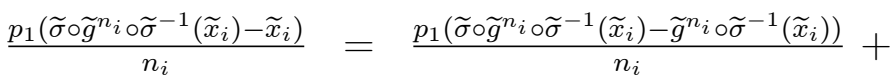

$$
\begin{aligned}
& \frac{p_{1}\left(\widetilde{g}^{n_{i}} \circ \widetilde{\sigma}^{-1}\left(\widetilde{x}_{i}\right)-\widetilde{\sigma}^{-1}\left(\widetilde{x}_{i}\right)\right)}{n_{i}}+\frac{p_{1}\left(\widetilde{\sigma}^{-1}\left(\widetilde{x}_{i}\right)-\widetilde{x}_{i}\right)}{n_{i}} .
\end{aligned}
$$

Pelo item (v) da Proposição 2.1, temos que

$$
\widetilde{\sigma}=I d+\Phi
$$

ou seja,

$$
\widetilde{\sigma}(\widetilde{x})-\widetilde{x}=\Phi(\widetilde{x})
$$

para todo $\widetilde{x} \in \widetilde{\mathbb{A}}$. Mas, como $\Phi$ é uma função periódica com respeito à primeira coordenada, teremos que $p_{1}(\widetilde{\sigma}-I d)$ será uma função limitada. Similarmente, $p_{1}\left(\widetilde{\sigma}^{-1}-I d\right)$ será uma função limitada também. Desse modo,

$$
\begin{array}{ll}
\lim _{i \rightarrow \infty} \frac{p_{1}\left(\widetilde{\sigma} \circ \widetilde{g}^{n_{i}} \circ \widetilde{\sigma}^{-1}\left(\widetilde{x}_{i}\right)-\widetilde{g}^{n_{i}} \circ \widetilde{\sigma}^{-1}\left(\widetilde{x}_{i}\right)\right)}{n_{i}}=0 \\
\lim _{i \rightarrow \infty} \frac{p_{1}\left(\widetilde{\sigma}^{-1}\left(\widetilde{x}_{i}\right)-\widetilde{x}_{i}\right)}{n_{i}} & =0
\end{array}
$$

e portanto,

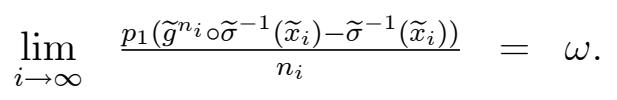

Em consequiência, $\omega \in \operatorname{Rot}(\widetilde{g})$, ou seja, $\operatorname{Rot}(\widetilde{f}) \subset \operatorname{Rot}(\widetilde{g})$. Do mesmo jeito pode ser demonstrado que $\operatorname{Rot}(\widetilde{g}) \subset \operatorname{Rot}(\widetilde{f})$. 


\subsection{A ordem cíclica no círculo e no anel}

Nesta seção veremos como a definição de ordem cíclica no círculo se estende para o anel.

Definição 2.11 Dados três pontos distintos $p_{1}, p_{2}, p_{3}$ em $\mathbb{S}^{1}$, a tripla $\left(p_{1}, p_{2}, p_{3}\right)$ chama-se positiva se o ponto $p_{2}$ pertence ao caminho que vai de $p_{1}$ a $p_{3}$ na direção positiva. Lembre-se que estamos utilizando no círculo a orientação induzida por $\mathbb{R}$.

Definição 2.12 Seja $\gamma:[0,1] \rightarrow \mathbb{A}$ um arco simples, então $\gamma$ é chamado de arco essencial simples se $\gamma$ une uma das componentes da fronteira com a outra e se $\gamma([0,1[)$ está incluído no interior de $\mathbb{A}$. Do mesmo jeito, definimos a noção de arco essencial simples no recobrimento universal $\widetilde{\mathbb{A}}$.

Desse modo, sejam $\gamma_{1}, \gamma_{2}, \gamma_{3}$ arcos essenciais simples dois a dois disjuntos e $p_{1}, p_{2}, p_{3}$ seus pontos extremos numa das componentes da fronteira, respectivamente. Então a tripla $\left(\gamma_{1}, \gamma_{2}, \gamma_{3}\right)$ é positiva se e somente se a tripla $\left(p_{1}, p_{2}, p_{3}\right)$ é positiva.

\subsection{Intervalos de Farey}

Um intervalo de Farey é um intervalo $] \frac{p}{q}, \frac{p^{\prime}}{q^{\prime}}[$ de $\mathbb{R}$ com extremos racionais, tais que $p^{\prime} q-p q^{\prime}=1$. Em nosso trabalho, todos os números racionais $\frac{p}{q}$ $(p \in \mathbb{Z}$ e $q \in \mathbb{N})$ serão escritos como frações irredutíveis.

A seguir, vamos mostrar que a ordem cíclica dos primeiros $q+q^{\prime}-1$ iterados de qualquer órbita de um ponto pela rotação rígida de ângulo $\alpha \in$ ]$\frac{p}{q}, \frac{p^{\prime}}{q^{\prime}}[$ não depende da escolha de $\alpha$.

Lema 2.3 Para qualquer $k \in\left\{1, \ldots, q+q^{\prime}-1\right\}$, o intervalo $] \frac{k \cdot p}{q}, \frac{k \cdot p^{\prime}}{q^{\prime}}[n \tilde{a} o$ contém inteiros. 
Demonstração. Seja $k$ um inteiro positivo tal que o intervalo $] \frac{k \cdot p}{q}, \frac{k \cdot p^{\prime}}{q^{\prime}}[$ contenha um inteiro $\ell$. Então o racional $\frac{\ell}{k}$ pertence ao intervalo $] \frac{p}{q}, \frac{p^{\prime}}{q^{\prime}}[$. Assim,

$$
\frac{p^{\prime}}{q^{\prime}}-\frac{p}{q}=\left(\frac{p^{\prime}}{q^{\prime}}-\frac{\ell}{k}\right)+\left(\frac{\ell}{k}-\frac{p}{q}\right)=\left(\frac{k p^{\prime}-\ell q^{\prime}}{k q^{\prime}}\right)+\left(\frac{\ell q-k p}{k q}\right) .
$$

Dado que $\ell$ é um inteiro então $\ell q-k p$ também vai ser um inteiro. Mas, $\ell q-k p>0$, o que implica que $\ell q-k p \geq 1$. Similarmente, $k p^{\prime}-\ell q^{\prime} \geq 1$. Portanto, $\frac{\ell q-k p}{k q} \geq \frac{1}{k q}$ e $\frac{k p^{\prime}-\ell q^{\prime}}{k q^{\prime}} \geq \frac{1}{k q^{\prime}}$. Juntando isto com o fato que $\frac{p^{\prime}}{q^{\prime}}-\frac{p}{q}=\frac{1}{q q^{\prime}}$ temos finalmente que

$$
\frac{1}{q q^{\prime}} \geq \frac{1}{k q}+\frac{1}{k q^{\prime}}=\frac{1}{k}\left(\frac{q+q^{\prime}}{q q^{\prime}}\right)
$$

ou seja, $k \geq q+q^{\prime}$.

O lema anterior, implica que para qualquer inteiro $k \in\left\{1, \ldots, q+q^{\prime}-1\right\}$ existe um único inteiro $n_{k}$ de tal modo que o intervalo $] k\left(\frac{p}{q}\right)-n_{k}, k\left(\frac{p^{\prime}}{q^{\prime}}\right)-n_{k}[$ está contido em ]0,1[. Tomemos um número $\alpha$ que pertença ao intervalo ]$\frac{p}{q}, \frac{p^{\prime}}{q^{\prime}}\left[\right.$ e definimos $\alpha_{k}=k \alpha-n_{k}$ para $k \in\left\{1, \ldots, q+q^{\prime}-1\right\}$. Temos assim, o seguinte resultado:

Proposição 2.3 A ordem dos $\alpha_{1}, \alpha_{2}, \ldots, \alpha_{q+q^{\prime}-1}$ não depende da escolha do número $\alpha$.

Demonstração. Sejam $k_{1}$ e $k_{2}$ inteiros em $\left\{1, \ldots, q+q^{\prime}-1\right\}$. Tomemos agora a diferença,

$$
\alpha_{k_{2}}-\alpha_{k_{1}}=\left(k_{2}-k_{1}\right) \alpha-\left(n_{k_{2}}-n_{k_{1}}\right) .
$$

Suponhamos que $\alpha_{k_{2}}-\alpha_{k_{1}}=0$, nesse caso, $\left(k_{2}-k_{1}\right) \alpha$ vai ser um número inteiro. Se $\alpha \in] \frac{p}{q}, \frac{p^{\prime}}{q^{\prime}}[$, pelo Lema 2.3 sabemos que o intervalo $] \frac{p}{q}, \frac{p^{\prime}}{q^{\prime}}[$ não 
contém racionais com denominador menor ou igual a $q+q^{\prime}-1$, ou seja, $\left(k_{2}-k_{1}\right) \alpha$ não pode ser um número inteiro, pois $k_{2}-k_{1}<q+q^{\prime}-1$, o que dá uma contradição. Assim, a diferença entre $\alpha_{k_{2}}$ e $\alpha_{k_{1}}$ não pode ser nula. Além disso, esta diferença depende continuamente de $\alpha$, portanto o sinal dela não depende do valor de $\alpha$.

Por último, os intervalos de Farey apresentam uma propriedade muito importante para o nosso estudo. É fácil ver que se $] \frac{p}{q}, \frac{p^{\prime}}{q^{\prime}}[$ é um intervalo de Farey, então $] \frac{p}{q}, \frac{p+p^{\prime}}{q+q^{\prime}}[\mathrm{e}] \frac{p+p^{\prime}}{q+q^{\prime}}, \frac{p^{\prime}}{q^{\prime}}\left[\right.$ também o são, com comprimentos $\frac{1}{q\left(q+q^{\prime}\right)} \mathrm{e}$ $\frac{1}{q\left(q+q^{\prime}\right)}$, respectivamente. Se continuamos esse processo mais uma vez, obtemos os intervalos de Farey

$$
] \frac{p}{q}, \frac{2 p+p^{\prime}}{2 q+q^{\prime}}[,] \frac{2 p+p^{\prime}}{2 q+q^{\prime}}, \frac{p+p^{\prime}}{q+q^{\prime}}[,] \frac{p+p^{\prime}}{q+q^{\prime}}, \frac{p+2 p^{\prime}}{q+2 q^{\prime}}[,] \frac{p+2 p^{\prime}}{q+2 q^{\prime}}, \frac{p^{\prime}}{q^{\prime}}[
$$

de comprimentos

$$
\frac{1}{q\left(2 q+q^{\prime}\right)}, \frac{1}{\left(q+q^{\prime}\right)\left(2 q+q^{\prime}\right)}, \frac{1}{\left(q+q^{\prime}\right)\left(q+2 q^{\prime}\right)} \text { e } \frac{1}{q^{\prime}\left(q+2 q^{\prime}\right)} \text {, respectivamente. }
$$

Podemos ver então que para qualquer número irracional positivo é possível encontrar um intervalo de Farey que o contém, tão pequeno quanto se queira. Por exemplo, se $\alpha$ é um número irracional maior do que zero, o primeiro intervalo de Farey poderia ser $] \frac{\lfloor\alpha\rfloor}{1}, \frac{\lfloor\alpha\rfloor+1}{1}[$, onde \lfloor\rfloor representa a função parte inteira de um número real. Desse modo, pode-se mostrar o seguinte fato:

Lema 2.4 Seja $\alpha$ um número irracional positivo. Então dado qualquer número inteiro positivo $n$, existe um intervalo de Farey $] \frac{p}{q}, \frac{p^{\prime}}{q^{\prime}}\left[\right.$ tal que $q, q^{\prime} \geq n$ $e$

$$
\left|\alpha-\frac{p}{q}\right|<\frac{1}{q^{2}} .
$$


Demonstração. Uma prova deste resultado pode ser encontrada em [13].

Nos próximos capítulos veremos a grande utilidade que têm as propriedades aritméticas dos intervalos de Farey nas demonstrações dos resultados principais deste trabalho.

Isto conclui a apresentação das preliminares. 


\section{Capítulo 3}

\section{O Teorema do 'arco de translação': Prova do Teorema 1.1}

No capítulo anterior, vimos que para qualquer número irracional $\alpha$ existe um

intervalo de Farey $] \frac{p}{q}, \frac{p^{\prime}}{q^{\prime}}\left[\right.$, onde $q$ e $q^{\prime}$ podem ser arbitrariamente grandes. Fazendo uso deste resultado, mudaremos as hipóteses do Teorema 1.1 que descreve o conjunto de rotação, e obteremos um novo teorema, de tal maneira que o Teorema 1.1 é um corolário deste novo resultado, que será denominado Teorema do 'arco de translação'. Assim, neste capítulo apresentaremos uma primeira prova do Teorema do 'arco de translação' usando só as propriedades aritméticas dos intervalos de Farey e algumas operações clássicas dos arcos essenciais simples do recobrimento $\widetilde{\mathbb{A}}$.

\subsection{Enunciado do Teorema do 'arco de translação'}

A afirmação que provaremos é: 
Teorema 3.1 (Teorema do 'arco de translação') Seja $h: \mathbb{A} \rightarrow \mathbb{A} u m$ homeomorfismo isotópico à identidade, e seja $\widetilde{h}: \widetilde{\mathbb{A}} \rightarrow \widetilde{\mathbb{A}}$ um levantamento de $h$. Suponhamos que o conjunto de rotação de $\widetilde{h}$ esteja contido num intervalo de Farey $] \frac{p}{q}, \frac{p^{\prime}}{q^{\prime}}[$. Então, existe um arco essencial simples $\gamma$ (ver Definição 2.12) no anel $\mathbb{A}$ tal que os arcos $\gamma, h(\gamma), \ldots, h^{q+q^{\prime}-1}(\gamma)$ são dois a dois disjuntos. Ainda, a ordem cíclica destes arcos em $\mathbb{A}$ será a mesma que a ordem cíclica dos primeiros $q+q^{\prime}-1$ iterados de um segmento vertical por qualquer rotação rígida de ângulo $\alpha \in] \frac{p}{q}, \frac{p^{\prime}}{q^{\prime}}[$, isto é, para quaisquer $k_{1}, k_{2}, k_{3} \in\left\{0, \ldots, q+q^{\prime}-1\right\}$, a tripla $\left(h^{k_{1}}(\gamma), h^{k_{2}}(\gamma), h^{k_{3}}(\gamma)\right)$ será positiva se e somente se a tripla $\left(k_{1} \alpha, k_{2} \alpha, k_{3} \alpha\right)$ for positiva, onde os números $k_{i} \alpha$ são considerados elementos do círculo $\mathbb{R} / \mathbb{Z}$.

Desse modo, vejamos como o resultado enunciado na introdução (Teorema 1.1) segue diretamente deste novo teorema.

Prova do Teorema 1.1. Suponhamos que o Teorema 3.1 esteja certo. Então, para qualquer inteiro positivo $n$, se o conjunto de rotação é um número irracional $\alpha$, pelo Lema 2.4, podemos encontrar um intervalo de Farey $] \frac{p}{q}, \frac{p^{\prime}}{q^{\prime}}[$ tal que $\alpha$ pertença a ele e $n \leq q+q^{\prime}-1$. Portanto, pelo Teorema 3.1, existe um arco essencial simples $\gamma$ no anel $\mathbb{A}$, tal que os $\operatorname{arcos} \gamma, h(\gamma), \ldots, h^{n}(\gamma)$ são dois a dois disjuntos. Mais ainda, a ordem cíclica destes $\operatorname{arcos}$ em $\mathbb{A}$ será a mesma que a ordem cíclica dos primeiros $n$ iterados de um segmento vertical pela rotação rígida de qualquer ângulo $\alpha \in] \frac{p}{q}, \frac{p}{q},[$.

Desse modo, o primeiro resultado de nosso trabalho está provado.

Antes de iniciarmos a primeira prova do Teorema 3.1, mais uma propriedade básica será descrita. 
Lema 3.1 Sejam $h$ um homeomorfismo do anel fechado $\mathbb{A}$, isotópico à identidade, $e \widetilde{h}: \widetilde{\mathbb{A}} \rightarrow \widetilde{\mathbb{A}}$ um levantamento de $h$. Suponhamos que o conjunto de rotação de $\widetilde{h}$ esteja contido em $] 0,+\infty[$, e escolhamos um número real $\rho$ tal que $0<\rho<\inf (\operatorname{Rot}(\widetilde{h}))$. Então, existe um número real s tal que para todo $\widetilde{x}$ em $\widetilde{\mathbb{A}}$ e para todo inteiro positivo $n$,

$$
p_{1}\left(\widetilde{h}^{n}(\widetilde{x})\right) \geq p_{1}(\widetilde{x})+\rho n-s .
$$

Demonstração. Suponhamos que a conclusão seja falsa. Então, para todo número real $s$ existe um $\widetilde{x}$ em $\widetilde{\mathbb{A}}$ e um inteiro positivo $n$ tais que

$$
p_{1}\left(\widetilde{h}^{n}(\widetilde{x})\right)<p_{1}(\widetilde{x})+\rho n-s .
$$

Desse modo, tomemos $s=i$ para $i \in \mathbb{N}$. Obtemos assim seqüências $\left(n_{i}\right)_{i=1}^{\infty}$ e $\left(\widetilde{x}_{i}\right)_{i=1}^{\infty}$ tais que

$$
\begin{aligned}
p_{1}\left(\widetilde{h}^{n_{i}}\left(\widetilde{x}_{i}\right)\right) & <p_{1}\left(\widetilde{x}_{i}\right)+\rho n_{i}-i, \\
\frac{p_{1}\left(\widetilde{h}^{n_{i}}\left(\widetilde{x}_{i}\right)-\widetilde{x}_{i}\right)}{n_{i}} & <\rho-\frac{i}{n_{i}} .
\end{aligned}
$$

No entanto, vimos anteriormente que $K_{k}(\widetilde{h}) \subset[-L, L]$ para todo $k \in \mathbb{N}$, onde $L>0$ tal que $K_{1}(\widetilde{h}) \subset[-L, L]$. Portanto, quando $i \rightarrow+\infty$ temos que $n_{i} \rightarrow+\infty$, e a seqüência $\left(\frac{p_{1}\left(\widetilde{h}^{n}\left(\widetilde{x}_{i}\right)-\widetilde{x}_{i}\right)}{n_{i}}\right)_{i=1}^{\infty}$ é limitada superiormente, o que implica que possui uma subseqüencia convergente cujo limite quando $i \rightarrow+\infty$ é menor ou igual a $\rho$. Assim chegamos a um absurdo, pois por hipótese, $0<\rho<\inf (\operatorname{Rot}(\widetilde{h}))$.

Corolário 3.1 Com as mesmas hipóteses do Lema 3.1, temos que para todo subconjunto compacto $K$ de $\widetilde{\mathbb{A}}$ e todo $s_{0} \in \mathbb{N}$, existe um $n_{0} \in \mathbb{Z}$ tal que para todo $n \geq n_{0}, \widetilde{h}^{n}(K) \subset\left[s_{0},+\infty[\times[0,1]\right.$. 
Demonstração. Seja $a=\min \left\{p_{1}(\widetilde{x}): \widetilde{x} \in K \subset \widetilde{\mathbb{A}}\right\}$. Dado que $K$ é um subconjunto compacto de $\widetilde{\mathbb{A}}$ então $a>-\infty$. Pelo Lema 3.1, para todo $\widetilde{x}$ em $K$ e para todo inteiro positivo $n$, temos que

$$
p_{1}\left(\widetilde{h}^{n}(\widetilde{x})\right) \geq a+\rho n-s .
$$

Assim, dado $s_{0}$ um número real, se $n_{0}=\left\lfloor\frac{s_{0}+s-a}{\rho}\right\rfloor+1$, então $p_{1}\left(\widetilde{h}^{n}(\widetilde{x})\right) \geq$ $s_{0}$ para todo inteiro $n \geq n_{0}$. Em outras palavras, $\widetilde{h}^{n}(K) \subset\left[s_{0},+\infty[\times[0,1]\right.$ para $n \geq n_{0}$.

\subsection{Primeira prova do Teorema do 'arco de translação'}

Para esta primeira prova, introduziremos mais algumas notações e propriedades que serão utilizadas ao longo da mesma e dos próximos capítulos.

Para todo arco essencial simples $\Gamma$ no recobrimento universal $\widetilde{\mathbb{A}}$, denotaremos por $R(\Gamma)$ o fecho da componente conexa de $\widetilde{\mathbb{A}} \backslash \Gamma$ que está 'à direita' do arco $\Gamma$.

Definição 3.1 Dado um arco essencial simples $\Gamma$ em $\widetilde{\mathbb{A}}$ e um homeomorfismo $\Psi: \widetilde{\mathbb{A}} \rightarrow \widetilde{\mathbb{A}}$, dizemos que o conjunto $R(\Gamma)$ é um atrator (resp. um atrator estrito) para $\Psi$ se a imagem de $R(\Gamma)$ por $\Psi$ está contida em $R(\Gamma)$ (resp. no interior de $R(\Gamma)$ ).

Notemos que se $\Psi$ é isotópico à identidade, então $R(\Psi(\Gamma))=\Psi(R(\Gamma))$ (lembramos que, pela Proposição 2.1, $\Psi$ preservará a orientação). Portanto, o conjunto $R(\Gamma)$ será um atrator (resp. estrito) para $\Psi$ se e somente se a imagem do arco $\Gamma$ por $\Psi$ estiver contida em $R(\Gamma)$ (resp. no interior de $R(\Gamma)$ ). 
Sejam $\Gamma_{1}$ e $\Gamma_{2}$ dois arcos essenciais simples, então diremos que $\Gamma_{1}<\Gamma_{2}$ se $\Gamma_{2} \subset \operatorname{Int}\left(R\left(\Gamma_{1}\right)\right)$ e $\Gamma_{1} \leq \Gamma_{2}$ se $\Gamma_{2} \subset R\left(\Gamma_{1}\right)$.

Observação 3.1 Sejam $\Gamma_{1}, \Gamma_{2} e \Gamma_{3}$ arcos essenciais simples em $\widetilde{\mathbb{A}}$. Então:

(i) Se $\Gamma_{1}<\Gamma_{2}$ (resp. $\left.\Gamma_{1} \leq \Gamma_{2}\right)$ e $\Gamma_{2}<\Gamma_{3}$ (resp. $\left.\Gamma_{2} \leq \Gamma_{3}\right)$ então $\Gamma_{1}<\Gamma_{3}$ (resp. $\Gamma_{1} \leq \Gamma_{3}$ ).

(ii) Se $\Gamma_{1}<\Gamma_{2}$ (resp. $\Gamma_{1} \leq \Gamma_{2}$ ), então $\Psi\left(\Gamma_{1}\right)<\Psi\left(\Gamma_{2}\right)$ (resp. $\Psi\left(\Gamma_{1}\right) \leq$ $\Psi\left(\Gamma_{2}\right)$ ) para todo homeomorfismo $\Psi: \widetilde{\mathbb{A}} \rightarrow \widetilde{\mathbb{A}}$ isotópico à identidade.

(iii) Seja $\Phi: \widetilde{\mathbb{A}} \rightarrow \widetilde{\mathbb{A}}$, um homeomorfismo isotópico à identidade e $\Gamma$ um arco essencial simples em $\widetilde{\mathbb{A}}$ tais que $\Gamma \cap \Phi(\Gamma)=\emptyset$. Então, o conjunto $\operatorname{Rot}(\Phi) \subset[0,+\infty[$, se e somente se, $\Gamma<\Phi(\Gamma)$.

Lema 3.2 Sejam $\Gamma_{1}$ e $\Gamma_{2}$ dois arcos essenciais simples em $\widetilde{\mathbb{A}}$, e seja $U$ a única componente conexa não limitada do conjunto $\left(\widetilde{\mathbb{A}} \backslash R\left(\Gamma_{1}\right)\right) \cap\left(\widetilde{\mathbb{A}} \backslash R\left(\Gamma_{2}\right)\right)$. Então a fronteira (em $\widetilde{\mathbb{A}}$ ) de $U$ é um arco essencial simples, o qual denotare$\operatorname{mos} \Gamma_{1} \bigvee \Gamma_{2}$

Demonstração. Para a demonstração deste lema utilizaremos o seguinte resultado clássico de Kerékjártó: Sejam $U_{1}$ e $U_{2}$ dois domínios de Jordan na esfera $S^{2}$ (note que um domínio de Jordan em $\mathbb{S}^{2}$ é um conjunto aberto tal que sua fronteira é homeomorfa ao círculo). Então cada componente conexa de $U_{1} \cap U_{2}$ é também um domínio de Jordan. Para uma prova deste fato, ver [16].

Para utilizar o fato anterior, o recobrimento universal $\widetilde{\mathbb{A}}$ será visto como um subconjunto de $\mathbb{R}^{2}$ e a esfera $\mathbb{S}^{2}=\mathbb{R}^{2} \cup\{\infty\}$ como a compactificação de um ponto. Portanto, é fácil ver que $\left(\widetilde{\mathbb{A}} \backslash R\left(\Gamma_{1}\right)\right) \cup\{\infty\}$ e $\left(\widetilde{\mathbb{A}} \backslash R\left(\Gamma_{2}\right)\right) \cup\{\infty\}$ são domínios de Jordan em $\mathbb{S}^{2}$. Assim, pelo resultado de Kerékjártó temos que $U$ será um domínio de Jordan em $\mathbb{S}^{2}$ cuja fronteira contém o ponto $\infty$ e 
é homeomorfa ao círculo. Dado que $\Gamma_{1} \bigvee \Gamma_{2}$ está contido na fronteira de $U$, então $\Gamma_{1} \bigvee \Gamma_{2}$ é um arco essencial simples (ver Figura 3.1).
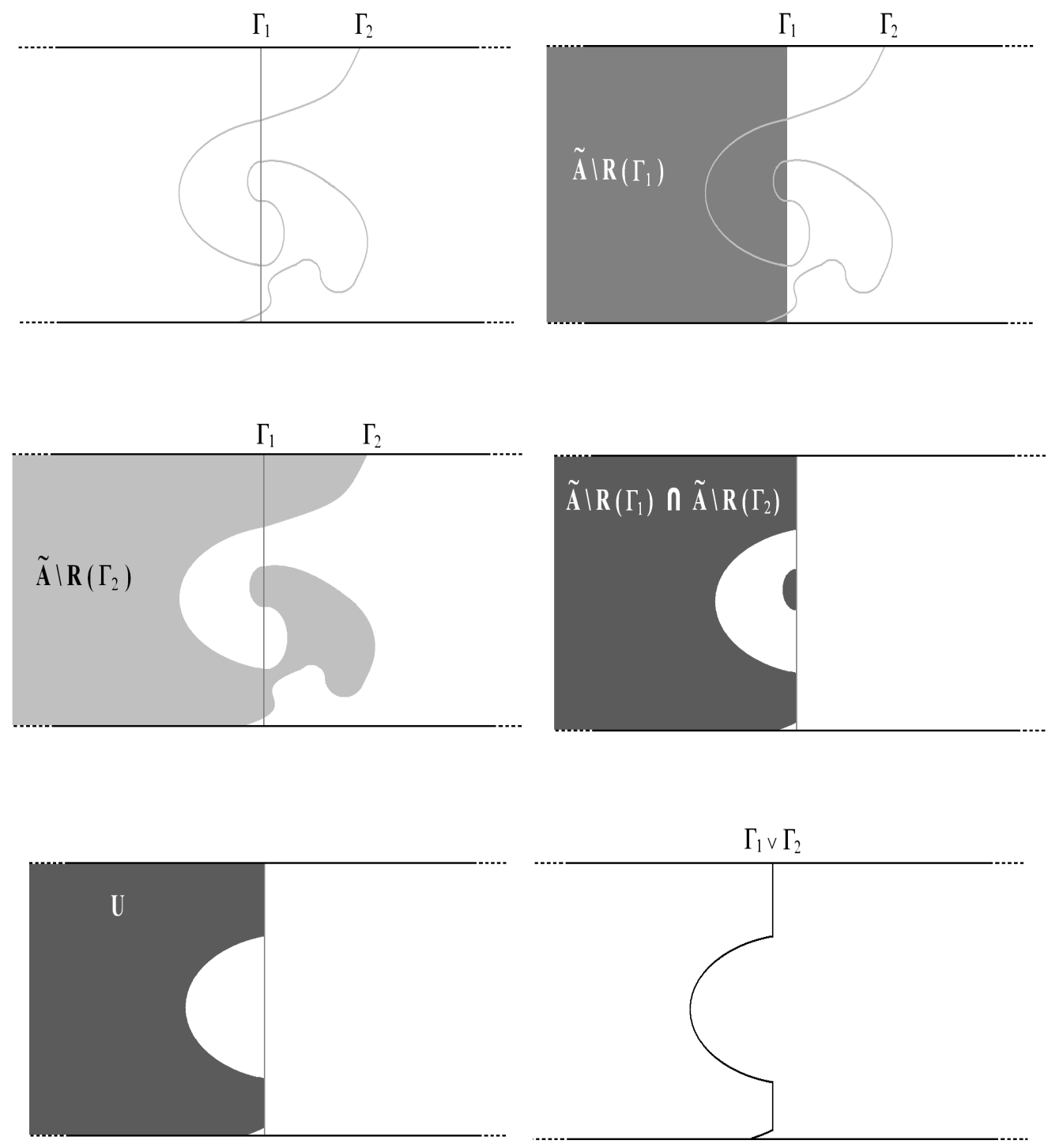

Figura 3.1: Arcos essenciais simples $\Gamma_{1}$ e $\Gamma_{2}$, e construção do arco $\Gamma_{1} \vee \Gamma_{2}$ 
Observação 3.2 Sejam $\Gamma_{1}$ e $\Gamma_{2}$ dois arcos essenciais simples em $\widetilde{\mathbb{A}}$, pela definição de $\Gamma_{1} \bigvee \Gamma_{2}$, temos que $\Gamma_{1} \bigvee \Gamma_{2} \subset \Gamma_{1} \cup \Gamma_{2}$. Similarmente, os conjuntos $R\left(\Gamma_{1}\right)$ e $R\left(\Gamma_{2}\right)$ estão contidos no conjunto $R\left(\Gamma_{1} \vee \Gamma_{2}\right)$. Então:

(i) Se $\Gamma_{3}$ é um arco essencial simples tal que $\Gamma_{3}<\Gamma_{1}$ e $\Gamma_{3}<\Gamma_{2}$, então $\Gamma_{3}<\Gamma_{1} \vee \Gamma_{2}$,

(ii) $\Gamma_{1} \bigvee \Gamma_{2} \leq \Gamma_{1}$ e $\Gamma_{1} \bigvee \Gamma_{2} \leq \Gamma_{2}$

(iii) $\Psi\left(\Gamma_{1} \vee \Gamma_{2}\right)=\Psi\left(\Gamma_{1}\right) \vee \Psi\left(\Gamma_{2}\right)$, para todo homeomorfismo $\Psi: \widetilde{\mathbb{A}} \rightarrow \widetilde{\mathbb{A}}$, isotópico à identidade.

Imediatamente notamos que a operação que associa a dois arcos essenciais simples $\Gamma_{1}, \Gamma_{2}$ o arco essencial simples $\Gamma_{1} \vee \Gamma_{2}$ é associativa e comutativa. Daí, dado qualquer número finito de arcos essenciais simples $\Gamma_{1}, \ldots, \Gamma_{n}$ o arco essencial simples $\Gamma_{1} \vee \ldots \vee \Gamma_{n}$ está bem definido.

Para simplificar a prova do Teorema 3.1, demonstraremos a seguinte proposição, de forma que, junto com as propriedades aritméticas dos intervalos de Farey, o Teorema 3.1 seguirá diretamente.

Proposição 3.1 Sejam $\Psi_{1}, \ldots, \Psi_{p}$ homeomorfismos do recobrimento universal $\widetilde{\mathbb{A}}$ isotópicos à identidade, que comutam dois a dois, e também comutam com a translação. Suponhamos que o conjunto de rotação de cada um deles está contido em $] 0,+\infty[$. Então existe um arco essencial simples $\Gamma$ em $\widetilde{\mathbb{A}}$, tal que o conjunto $R(\Gamma)$ é um atrator estrito para cada um dos homeomorfismos $\Psi_{1}, \ldots, \Psi_{p}$

Demonstração. Nesta prova, aplicaremos o princípio da indução junto com as operações dos arcos essenciais simples, isto significa que para todo $k \in\{1, \ldots, p\}$, vamos construir um arco essencial simples $\Gamma_{k}$ tal que o conjunto $R\left(\Gamma_{k}\right)$ seja um atrator estrito para os homeomorfismos $\Psi_{1}, \ldots, \Psi_{k}$. 
- Para $k=1$ :

Seja $\Gamma_{0}$ um arco essencial simples em $\widetilde{\mathbb{A}}$. Dado que $\Gamma_{0}$ é um conjunto compacto, pelo Corolário 3.1, existe um inteiro $N$ tal que $\Gamma_{0}<\Psi_{1}^{N}\left(\Gamma_{0}\right)$, o qual implica que $\Psi_{1}^{-N}\left(\Gamma_{0}\right)<\Gamma_{0}$. Consideremos os seguintes arcos essenciais simples $\Lambda_{0}, \ldots, \Lambda_{N-1}$ tais que

$$
\Psi_{1}^{-N}\left(\Gamma_{0}\right)<\Lambda_{N-1}<\Lambda_{N-2}<\cdots<\Lambda_{1}<\Lambda_{0}=\Gamma_{0},
$$

(ver Figura 3.2) e definimos

$$
\Gamma_{1}:=\Lambda_{0} \vee \Psi_{1}\left(\Lambda_{1}\right) \vee \cdots \vee \Psi_{1}^{N-1}\left(\Lambda_{N-1}\right)=\bigvee_{i=0}^{N-1} \Psi_{1}^{i}\left(\Lambda_{i}\right)
$$

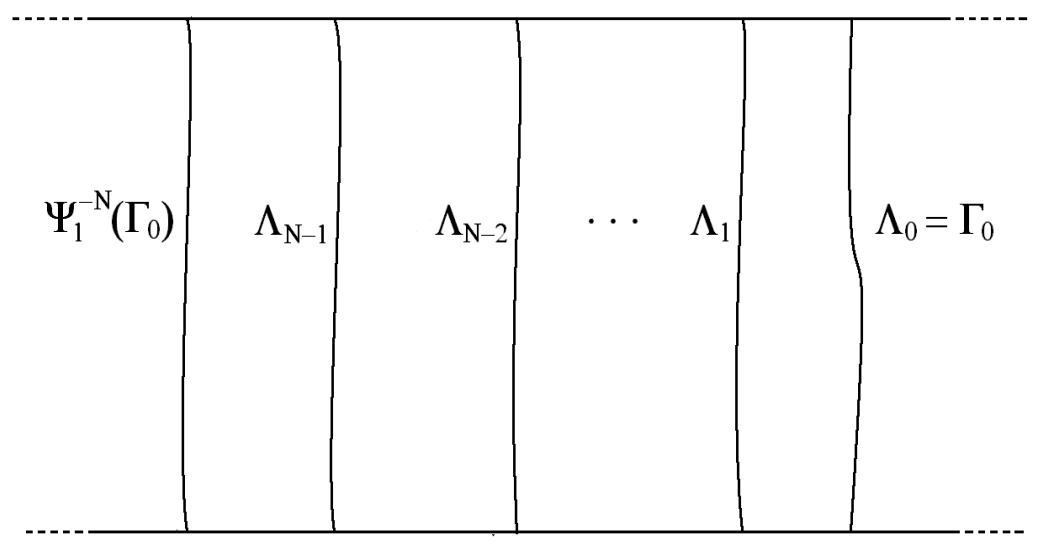

Figura 3.2: Arcos essenciais simples $\Lambda_{0}, \ldots, \Lambda_{N-1}$

Para $i \in\{0, \ldots, N-2\}$, vemos que $\Gamma_{1} \leq \Psi_{1}^{i+1}\left(\Lambda_{i+1}\right)$ (pelo item (ii) da Observação 3.2), e $\Psi_{1}^{i+1}\left(\Lambda_{i+1}\right)<\Psi_{1}^{i+1}\left(\Lambda_{i}\right)$ (pois $\Lambda_{i+1}<\Lambda_{i}$ ). Logo,

$$
\Gamma_{1}<\Psi_{1}^{i+1}\left(\Lambda_{i}\right) \text { para } i \in\{0, \ldots, N-2\} .
$$

Ainda, $\Gamma_{1} \leq \Lambda_{0}$ e $\Lambda_{0}=\Gamma_{0}<\Psi_{1}^{N}\left(\Lambda_{N-1}\right)\left(\right.$ pois $\left.\Psi_{1}^{-N}\left(\Gamma_{0}\right)<\Lambda_{N-1}\right)$. Portanto, 


$$
\Gamma_{1}<\Psi_{1}^{N}\left(\Lambda_{N-1}\right)
$$

Finalmente, pelo item (i) e (iii) da Observação 3.2, obtemos

$$
\Gamma_{1}<\bigvee_{i=0}^{N-1} \Psi_{1}^{i+1}\left(\Lambda_{i}\right)=\Psi\left(\Gamma_{1}\right)
$$

Desta última expressão temos que $R\left(\Gamma_{1}\right)$ é um atrator estrito para $\Psi_{1}$.

- Para $k \in\{1, \ldots, p-1\}$ :

Suponhamos que existe um arco essencial simples $\Gamma_{k}$ em $\widetilde{\mathbb{A}}$, tal que o conjunto $R\left(\Gamma_{k}\right)$ é um atrator estrito para cada um dos homeomorfismos $\Psi_{1}, \ldots, \Psi_{k}$. Em outras palavras, $\Gamma_{k}<\Psi_{j}\left(\Gamma_{k}\right)$ para todo $j \leq k$. Como no caso anterior, existe um inteiro $N$ tal que $\Gamma_{k}<\Psi_{k+1}^{N}\left(\Gamma_{k}\right)$. Consideremos os seguintes arcos essenciais simples $\Lambda_{0}, \ldots, \Lambda_{N-1}$ tais que

$$
\Psi_{k+1}^{-N}\left(\Gamma_{k}\right)<\Lambda_{N-1}<\Lambda_{N-2}<\cdots<\Lambda_{1}<\Lambda_{0}=\Gamma_{k}
$$

e definimos

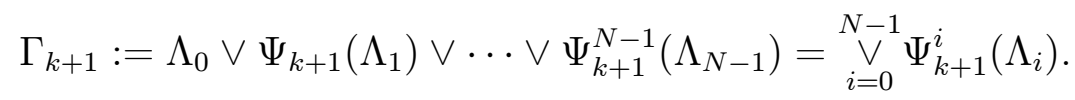

Do mesmo jeito que foi mostrado que $R\left(\Gamma_{1}\right)$ era um atrator estrito para $\Psi_{1}$, é possível mostrar que $R\left(\Gamma_{k+1}\right)$ é um atrator estrito para $\Psi_{k+1}$.

Resta apenas verificar que $R\left(\Gamma_{k+1}\right)$ também é um atrator estrito para $\Psi_{j}$ se $j \leq k$. Dado que $\Gamma_{k}<\Psi_{j}\left(\Gamma_{k}\right)$, então $\Psi_{j}^{-1}\left(\Gamma_{k}\right)<\Gamma_{k}$. Assim, podemos escolher os arcos $\Lambda_{0}, \ldots, \Lambda_{N-1}$ de tal modo que, para todo $j \in\{1, \ldots, k\}$,

$$
\Psi_{j}^{-1}\left(\Gamma_{k}\right)<\Lambda_{N-1}<\Lambda_{N-2}<\cdots<\Lambda_{1}<\Lambda_{0}=\Gamma_{k}
$$

Fixemos $j \in\{1, \ldots, k\}$. Por hipótese, sabemos que $\Psi_{j}$ comuta com $\Psi_{k+1}$, então para cada $i \in\{0, \ldots, N-1\}$, temos que 


$$
\Psi_{j}^{-1}\left(\Lambda_{i}\right) \leq \Psi_{j}^{-1}\left(\Gamma_{k}\right)<\Lambda_{i}
$$

e

$$
\Psi_{j}^{-1}\left(\Psi_{k+1}^{i}\left(\Lambda_{i}\right)\right)<\Psi_{k+1}^{i}\left(\Lambda_{i}\right)
$$

Agora, pelo item (ii) da Observação 3.2, temos que $\Gamma_{k+1} \leq \Psi_{k+1}^{i}\left(\Lambda_{i}\right)$, portanto,

$$
\Psi_{j}^{-1}\left(\Gamma_{k+1}\right) \leq \Psi_{j}^{-1}\left(\Psi_{k+1}^{i}\left(\Lambda_{i}\right)\right)
$$

Dessa forma, para todo $i \in\{0, \ldots, N-1\}$,

$$
\Psi_{j}^{-1}\left(\Gamma_{k+1}\right)<\Psi_{k+1}^{i}\left(\Lambda_{i}\right)
$$

Finalmente, pelo item (i) da Observação 3.2,

$$
\Psi_{j}^{-1}\left(\Gamma_{k+1}\right)<\bigvee_{i=0}^{N-1} \Psi_{k+1}^{i}\left(\Lambda_{i}\right)=\Gamma_{k+1}
$$

A expressão acima implica que $\Gamma_{k+1}<\Psi_{j}\left(\Gamma_{k+1}\right)$, em outras palavras, $R\left(\Gamma_{k+1}\right)$ é um atrator estrito para $\Psi_{j}$.

Estamos prontos para a prova do Teorema 3.1.

Prova do Teorema 3.1. A idéia desta demonstração consiste em aplicar a Proposição 3.1 a uma família bem escolhida de homeomorfismos, obtida pela composição de potências de $\tilde{h}$ e de potências de $T$, e assim conseguir o arco essencial simples que satisfaz as propriedades desejadas.

Seja $\rho$ qualquer número contido no intervalo de Farey $] \frac{p}{q}, \frac{p^{\prime}}{q^{\prime}}[$. As propriedades dos intervalos de Farey, vistas no Lema 2.3 e na Proposição 2.3, implicam que: 
- Para todo $k \in\left\{1, \ldots, q+q^{\prime}-1\right\}$, o número $k \cdot \rho$ não é um inteiro, permitindo assim definir os números $\left.\alpha_{k} \in\right] 0,1\left[\right.$ e os inteiros $n_{k}$ tais que $k \rho=n_{k}+\alpha_{k}$.

- Os números $\alpha_{1}, \ldots, \alpha_{q+q^{\prime}-1}$ são distintos, o que permite definir a permutação $\sigma$ no conjunto $\left\{1, \ldots, q+q^{\prime}-1\right\}$, tal que

$0<\sigma(1) \cdot \rho-n_{\sigma(1)}<\sigma(2) \cdot \rho-n_{\sigma(2)}<\ldots<\sigma\left(q+q^{\prime}-1\right) \cdot \rho-n_{\sigma\left(q+q^{\prime}-1\right)}<1$.

- Os inteiros $n_{1}, \ldots, n_{q+q^{\prime}-1}$ e a permutação $\sigma$ de fato não dependem da escolha do número $\rho$ contido em $] \frac{p}{q}, \frac{p^{\prime}}{q^{\prime}}[$.

Então, para todo $k \in\left\{1, \ldots, q+q^{\prime}-1\right\}$, definimos os homeomorfismos

$$
\Phi_{k}:=\widetilde{h}^{\sigma(k)} \circ T^{-n_{\sigma(k)}} .
$$

Ainda,

$$
\begin{gathered}
\Phi_{0}:=\mathrm{Id}, \\
\Phi_{q+q^{\prime}}:=T .
\end{gathered}
$$

Similarmente, para todo $k \in\left\{1, \ldots, q+q^{\prime}\right\}$, definimos os homeomorfismos

$$
\Psi_{k}=\Phi_{k} \circ \Phi_{k-1}^{-1} .
$$

Aplicando os itens (iii) e (iv) da Proposição 2.1 é fácil ver que os homeomorfismos $\Psi_{1}, \ldots, \Psi_{q+q^{\prime}}$ comutam com a translação $T$ e que comutam dois a dois.

Seja $k \in\left\{2, \ldots, q+q^{\prime}-1\right\}$. Temos que

$$
\Psi_{k}=\widetilde{h}^{\sigma(k)} \circ T^{-n_{\sigma(k)}} \circ T^{n_{\sigma(k-1)}} \circ \widetilde{h}^{-\sigma(k-1)}=\widetilde{h}^{\sigma(k)-\sigma(k-1)} \circ T^{-n_{\sigma(k)}+n_{\sigma(k-1)}} .
$$


Pelo Lema 2.2, o conjunto de rotação do homeomorfismo $\Psi_{k}$ é:

$$
\operatorname{Rot}\left(\Psi_{k}\right)=\left\{\sigma(k) \cdot \rho-n_{\sigma(k)}-\left(\sigma(k-1) \cdot \rho-n_{\sigma(k-1)}\right): \rho \in \operatorname{Rot}(\widetilde{h})\right\}
$$

Como $\operatorname{Rot}(\widetilde{h})$ está contido no intervalo $] \frac{p}{q}, \frac{p^{\prime}}{q^{\prime}}[$, a definição da permutação $\sigma$ implica que $\operatorname{Rot}\left(\Psi_{k}\right)$ está contido em $] 0,+\infty[$.

Analogamente,

$$
\begin{aligned}
\operatorname{Rot}\left(\Psi_{1}\right) & =\left\{\sigma(1) \cdot \rho-n_{\sigma(1)}: \rho \in \operatorname{Rot}(\widetilde{h})\right\} \text { e } \\
\operatorname{Rot}\left(\Psi_{q+q^{\prime}}\right) & =\left\{1-\left(\sigma\left(q+q^{\prime}-1\right) \cdot \rho-n_{\sigma\left(q+q^{\prime}-1\right)}\right): \rho \in \operatorname{Rot}(\widetilde{h}) .\right.
\end{aligned}
$$

Estes conjuntos também estão contidos em $] 0,+\infty[$. Em conseqüência, os homeomorfismos $\Psi_{1}, \ldots, \Psi_{q+q^{\prime}}$ satisfazem as hipóteses da Proposição 3.1. Portanto, existe um arco essencial simples $\Gamma$ no recobrimento universal $\widetilde{\mathbb{A}}$ tal que $R(\Gamma)$ é um atrator estrito para cada um dos homeomorfismos $\Psi_{1}, \ldots, \Psi_{q+q^{\prime}}$. Seja $\gamma$ a projeção do arco $\Gamma$ no anel $\mathbb{A}$. Em particular, $\gamma$ também é um arco essencial simples no anel $\mathbb{A}$. Para mostrar isso, é suficiente que $\Gamma$ seja disjunto de sua imagem por $T$, caso contrário, $\gamma$ terá auto-interseções. Mas isto é simples de ver, pois

$$
T=\Psi_{q+q^{\prime}} \circ \ldots \circ \Psi_{1}
$$

e portanto $R(\Gamma)$ será um atrator estrito para a translação $T$, ou seja, $\Gamma \cap$ $T(\Gamma)=\emptyset$.

Agora, o ponto seguinte será provar que os $\operatorname{arcos} \gamma, h(\gamma), \ldots, h^{q+q^{\prime}-1}(\gamma)$ são disjuntos dois a dois. Em primeiro lugar, $R(\Gamma)$ é um atrator estrito para o homeomorfismo $\Psi_{1}=\Phi_{1}$, ou seja, $\Gamma<\Phi_{1}(\Gamma)$. Para todo $k \in\left\{2, \ldots, q+q^{\prime}-1\right\}$ sabemos que $R(\Gamma)$ é um atrator estrito para o homeomorfismo $\Psi_{k}$. Em outras palavras, 


$$
\Gamma<\Psi_{k}(\Gamma)
$$

Dado que $\Psi_{k}$ e $\Phi_{k-1}$ comutam (ver itens (iii) e (iv) da Proposição 2.1),

$$
\Phi_{k-1}(\Gamma)<\Psi_{k} \circ \Phi_{k-1}(\Gamma)=\Phi_{k}(\Gamma)
$$

Similarmente,

$$
\Phi_{q+q^{\prime}-1}(\Gamma)<\Psi_{q+q^{\prime}} \circ \Phi_{q+q^{\prime}-1}(\Gamma)=T(\Gamma) .
$$

Desse modo, obtemos que $\Gamma<\Phi_{1}(\Gamma)<\ldots<\Phi_{q+q^{\prime}-1}(\Gamma)<T(\Gamma)$ (ver Figura 3.3).

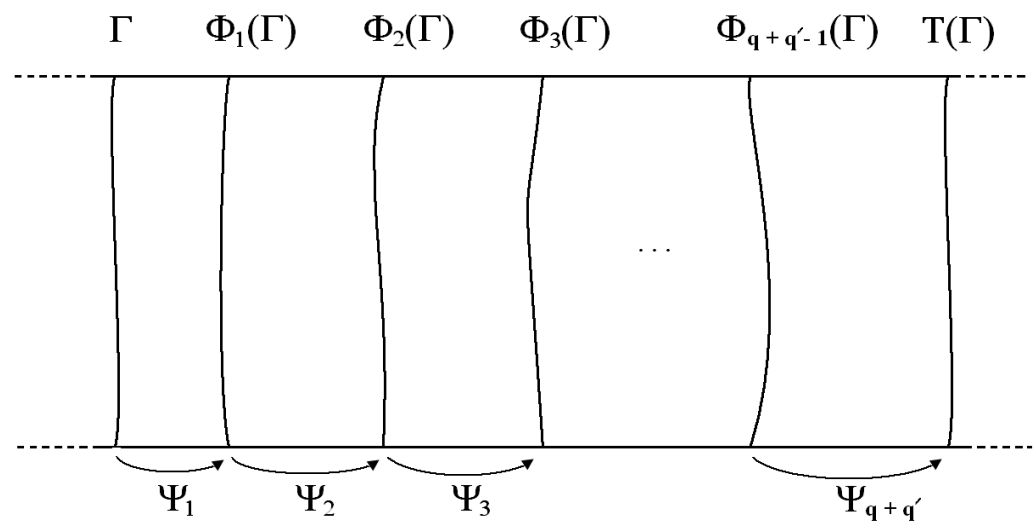

Figura 3.3: $\operatorname{Arcos} \Phi_{1}(\Gamma), \ldots, \Phi_{q+q^{\prime}-1}(\Gamma)$ e $T(\Gamma)$

Podemos então definir o conjunto $D:=\operatorname{Int}(R(\Gamma)) \backslash R(T(\Gamma))$. Dado que $D$ é um domínio fundamental para a aplicação de recobrimento $\widetilde{\pi}: \widetilde{\mathbb{A}} \rightarrow \mathbb{A}$, as projeções dos $\operatorname{arcos} \Gamma<\Phi_{1}(\Gamma)<\ldots<\Phi_{q+q^{\prime}-1}(\Gamma)$ são disjuntas duas a duas no anel $\mathbb{A}$. Pela definição do levantamento, para cada $k \in\left\{1, \ldots ., q+q^{\prime}-1\right\}$ os homeomorfismos $\Phi_{k}$ são um levantamento do homeomorfismo $h^{\sigma(k)}$, ainda, o arco $h^{\sigma(k)}(\gamma)$ será a projeção do arco $\Phi_{k}(\Gamma)$. Assim, fica demostrado que os $\operatorname{arcos} \gamma, h^{\sigma(1)}(\gamma), \ldots, h^{\sigma\left(q+q^{\prime}-1\right)}(\gamma)$ são dois a dois disjuntos. 
Resta só analisar a ordem cíclica dos $\operatorname{arcos} \gamma, h(\gamma), \ldots, h^{q+q^{\prime}-1}(\gamma)$ no anel A. Em particular, para quaisquer inteiros $k_{1}, k_{2}, k_{3} \in\left\{1, \ldots, q+q^{\prime}-1\right\}$ distintos dois a dois, temos que a tripla $\left(h^{k_{1}}(\gamma), h^{k_{2}}(\gamma), h^{k_{3}}(\gamma)\right)$ é positiva se e somente se as triplas $\left(\gamma, h^{k_{1}}(\gamma), h^{k_{2}}(\gamma)\right)$ e $\left(\gamma, h^{k_{2}}(\gamma), h^{k_{3}}(\gamma)\right)$ são positivas. Similarmente, a tripla $\left(k_{1} \rho, k_{2} \rho, k_{3} \rho\right)$ é positiva se e somente se as triplas $\left(0, k_{1} \rho, k_{2} \rho\right)$ é $\left(0, k_{2} \rho, k_{3} \rho\right)$ são positivas. Assim, a tripla $\left(\gamma, h^{k_{1}}(\gamma), h^{k_{2}}(\gamma)\right)$ é positiva se e somente se $\sigma^{-1}\left(k_{1}\right)<\sigma^{-1}\left(k_{2}\right)$. Pela definição de $\sigma$, isto implica que $k_{1} \rho-n_{k_{1}}<k_{2} \rho-n_{k_{2}}$, o que significa que a tripla $\left(0, k_{1} \rho, k_{2} \rho\right)$ no círculo $\mathbb{R} / \mathbb{Z}$ é positiva. Daí concluímos que os $\operatorname{arcos} \gamma, h(\gamma), \ldots, h^{q+q^{\prime}-1}(\gamma)$ estão na mesma ordem cíclica que o segmento vertical e seus primeiros $q+q^{\prime}-1$ iterados por qualquer rotação rígida de um ângulo $\rho \in] \frac{p}{q}, \frac{p^{\prime}}{q^{\prime}}[$.

Isto termina a primeira prova do Teorema do 'arco de translação'. No próximo capítulo daremos uma segunda prova deste mesmo resultado. 


\section{Capítulo 4}

\section{Uma segunda prova do Teorema do 'arco de translação'}

Neste capítulo apresentamos uma prova alternativa para o Teorema 3.1 (Teorema do 'arco de translação'). Usaremos dois argumentos independentes. O primeiro aplica principalmente as propriedades aritméticas dos intervalos de Farey e mostra que é suficiente encontrar um arco essencial simples cujas imagens pelas aplicações $\Phi_{1}=T^{-p} \circ \widetilde{h}^{q}$ e $\Phi_{2}=T^{-p^{\prime}} \circ \widetilde{h}^{q^{\prime}}$ são disjuntas. $\mathrm{O}$ segundo argumento, constrói o arco essencial simples da seguinte maneira: Suponha que temos dois homeomorfismos no recobrimento universal $\widetilde{\mathbb{A}}$ que comutam e considere sequências obtidas começando em qualquer ponto de $\widetilde{\mathbb{A}}$, iterando cada vez por um dos dois homeomorfismos. Provamos que se o conjunto de rotação desses homeomorfismos são ambos positivos, então todas as seqüências obtidas desse modo têm um deslocamento esquerdo limitado universalmente. A seguir demonstraremos que esse fato se mantem caso consideremos pseudo-órbitas em vez de órbitas, isto é, um pequeno erro em cada iterado é permitido. Finalmente, utilizando a descomposição por tijolos, que é um tipo de triangularização que produz atratores de uma maneira dire- 
ita devido ao comportamento controlado das pseudo-órbitas, conseguimos o arco essencial simples. Esta descomposição foi introduzida por Flucher (ver [8]). São conhecidas outras aplicações da decomposição por tijolos, que por exemplo podem ser vistas em [15].

\subsection{Prova alternativa}

Nesta seção descrevemos os dois resultados que foram apresentados no início deste capítulo, e que serão demonstrados nas próximas seções.

Proposição 4.1 Seja $\widetilde{h}: \widetilde{\mathbb{A}} \rightarrow \widetilde{\mathbb{A}}$ um levantamento de um homeomorfismo $h$ do anel $\mathbb{A}$ que é isotópico à identidade e seja $\Gamma \subset \widetilde{\mathbb{A}}$ um arco essencial simples em $\widetilde{\mathbb{A}}$. Suponha que existe um intervalo de Farey $] \frac{p}{q}, \frac{p^{\prime}}{q^{\prime}}[$ tal que (usando a notação da Seção 3.2)

$$
T^{-p^{\prime}} \circ \widetilde{h}^{q^{\prime}}(\Gamma)<\Gamma<T^{-p} \circ \widetilde{h}^{q}(\Gamma) .
$$

Então:

(i) Os $\operatorname{arcos} T^{-\ell} \circ \widetilde{h}^{k}(\Gamma)$, com $k \in\left\{0, \ldots, q+q^{\prime}-1\right\}$ e $\ell \in \mathbb{Z}$, são dois a dois disjuntos.

(ii) Os arcos estão ordenados em $\widetilde{\mathbb{A}}$ igual aos levantamentos dos primeiros $q+q^{\prime}-1$ iterados de um segmento vertical em $\mathbb{A}$ pela rotação $R_{\alpha}$ para qualquer $\alpha \in] \frac{p}{q}, \frac{p}{q},[$. Mais precisamente: dados dois pares de inteiros $(k, \ell)$ e $\left(k^{\prime}, \ell^{\prime}\right)$ em $\left\{0, \ldots, q+q^{\prime}-1\right\} \times \mathbb{Z}$, temos que

$$
T^{-\ell} \circ \widetilde{h}^{k}(\Gamma)<T^{-\ell^{\prime}} \circ \widetilde{h}^{k^{\prime}}(\Gamma) \Longleftrightarrow k \alpha-\ell<k^{\prime} \alpha-\ell^{\prime} .
$$


Em particular, o arco $\Gamma$ é disjunto do arco $T^{\ell}(\Gamma)$ para qualquer $\ell \in$ $\mathbb{Z}$. Portanto, $\gamma=\widetilde{\pi}(\Gamma)$ é um arco essencial simples em $\mathbb{A}$, disjunto dos seus $q+q^{\prime}-1$ primeiros iterados por $h$. Ainda, a ordem cíclica dos arcos $\gamma, h(\gamma), \ldots, h^{q+q^{\prime}-1}(\gamma)$ é a mesma que a dos iterados de um segmento vertical pela rotação de ângulo $\alpha$, para qualquer $\alpha$ pertencente ao intervalo de Farey ]$\frac{p}{q}, \frac{p^{\prime}}{q^{\prime}}[$.

Proposição 4.2 Sejam $\Phi_{1}, \Phi_{2}$ dois homeomorfismos de $\widetilde{\mathbb{A}}$, isotópicos à identidade, que comutam entre si e com a translação $T$, tais que seu conjunto de rotação está contido em $] 0,+\infty[$. Então, existe um arco essencial simples $\Gamma$ que é disjunto de $\Phi_{1}(\Gamma)$ e $\Phi_{2}(\Gamma)$.

Vejamos agora como o Teorema 3.1 segue destas duas proposições.

Segunda Prova do Teorema 3.1. Seja $\widetilde{h}$ como no Teorema 3.1. Consideremos as aplicações $\Phi_{1}:=T^{-p} \circ \widetilde{h}^{q}$ e $\Phi_{2}:=T^{p^{\prime}} \circ \widetilde{h}^{-q^{\prime}}$. Dado que o conjunto $\operatorname{Rot}(\widetilde{h})$ está contido no intervalo de Farey $] \frac{p}{q}, \frac{p^{\prime}}{q^{\prime}}[$, pelo Lema 2.2, segue que o conjunto de rotação de ambos homeomorfismos estão contidos em $] 0,+\infty[$. Utilizando a Proposição 4.2, obtemos um arco essencial simples $\Gamma$ que não intersecta $\Phi_{1}(\Gamma)$ e $\Phi_{2}(\Gamma)$. Como o conjunto de rotação é positivo para os dois homeomorfismos, então $\Phi_{2}^{-1}(\Gamma)<\Gamma<\Phi_{1}(\Gamma)$ (ver item (iii) da Observação 3.1). Podemos aplicar agora a Proposição 4.1.

Assim, seja $\gamma=\widetilde{\pi}(\Gamma)$. Pelo primeiro ponto da Proposição 4.1, temos que o arco $\Gamma$ é disjunto do arco $T^{\ell}(\Gamma)$ para todo $\ell \in \mathbb{Z}$, daí $\gamma$ é arco essencial simples em $\mathbb{A}$. Por outro lado, os $\operatorname{arcos} \gamma, h(\gamma), \ldots, h^{q+q^{\prime}-1}(\gamma)$ são dois a dois disjuntos. De fato, se $h^{k_{1}}(\gamma) \cap h^{k_{2}}(\gamma) \neq \emptyset$ para $k_{1}, k_{2} \in\left\{0, \ldots, q+q^{\prime}-1\right\}$, então existem inteiros $\ell_{1}, \ell_{2}$ tais que $T^{-\ell_{1}} \circ \widetilde{h}^{k_{1}}(\Gamma) \cap T^{-\ell_{2}} \circ \widetilde{h}^{k_{2}}(\Gamma) \neq \emptyset$, o qual contradiz a Proposição 4.1. 
Finalmente, dados $k, k^{\prime} \in\left\{0, \ldots, q+q^{\prime}-1\right\}$, suponhamos que a tripla $\left(\gamma, h^{k}(\gamma), h^{k^{\prime}}(\gamma)\right)$ seja positiva. Portanto,

$$
\Gamma<T^{-\ell} \circ \widetilde{h}^{k}(\Gamma)<T^{-\ell^{\prime}} \circ \widetilde{h}^{k^{\prime}}(\Gamma)<T(\Gamma)
$$

para $\ell, \ell^{\prime} \in \mathbb{Z}$. Pelo segundo ponto da Proposição 4.1, isto é equivalente a

$$
0<k \alpha-\ell<k^{\prime} \alpha-\ell^{\prime}<1 .
$$

Resulta então que a ordem cíclica dos $\operatorname{arcos} \gamma, h(\gamma), \ldots, h^{q+q^{\prime}-1}(\gamma)$ é a mesma que a ordem cíclica dos iterados de um segmento vertical pela rotação de ângulo $\alpha$, para qualquer $\alpha \in] \frac{p}{q}, \frac{p^{\prime}}{q^{\prime}}[$.

Isto termina a segunda prova do Teorema 3.1.

No resto deste capítulo vamos demonstrar as Proposições 4.1 e 4.2 .

\subsection{Prova da Proposição 4.1}

Nesta seção vamos demonstrar a Proposição 4.1. Para isso, utilizaremos as propriedades aritméticas dos intervalos de Farey e suas aproximações racionais, assim como o fato de que $T$ e $\widetilde{h}$ comutam. Além disso, duas afirmações serão demonstradas para simplificar a prova desta proposição.

Comecemos definindo as aproximações racionais dos intervalos de Farey.

Definição 4.1 Seja I $=] \frac{p}{q}, \frac{p^{\prime}}{q^{\prime}}[$ qualquer intervalo de Farey. Então, uma seqüência finita de números racionais $\left(\frac{p_{n}}{q_{n}}\right)_{1 \leq n \leq n_{0}}$, onde $p_{n}$ e $q_{n}$ são primos entre si, está associada a este intervalo se satisfaz as seguintes propriedades:

- $\frac{p_{1}}{q_{1}} e \frac{p_{2}}{q_{2}}=\frac{p_{1}}{q_{1}}+1$ são dois inteiros consecutivos.

- Para $2 \leq n \leq n_{0}-1$, existe $a_{n+1} \in \mathbb{N} \backslash\{0\}$ tal que 


$$
p_{n+1}=a_{n+1} p_{n}+p_{n-1} \quad e \quad q_{n+1}=a_{n+1} q_{n}+q_{n-1}
$$

- $\frac{p_{n_{0}-1}}{q_{n_{0}-1}}$ e $\frac{p_{n_{0}}}{q_{n_{0}}}$ são os extremos do intervalo de Farey I, ou seja,

$$
] \frac{p}{q}, \frac{p^{\prime}}{q^{\prime}}[=] \frac{p_{n_{0}-1}}{q_{n_{0}-1}}, \frac{p_{n_{0}}}{q_{n_{0}}}[\quad \text { ou } \quad] \frac{p}{q}, \frac{p^{\prime}}{q^{\prime}}[=] \frac{p_{n_{0}}}{q_{n_{0}}}, \frac{p_{n_{0}-1}}{q_{n_{0}-1}}[\text {. }
$$

Os números racionais $\frac{p_{1}}{q_{1}}, \ldots, \frac{p_{n_{0}}}{q_{n_{0}}}$ chamam-se aproximações comuns de Farey dos elementos do intervalo $I$.

Se queremos que (4.1) esteja definida também para $n=1$, escrevemos $\left(p_{0}, q_{0}\right)=(1,0)$ e $a_{2}=1$.

Por outro lado, com as mesmas hipóteses da Proposição 4.1, vamos introduzir a seguinte notação:

- Para todo $(\ell, k) \in \mathbb{Z}^{2}$, denotamos por $\Gamma(\ell, k)$ a curva $T^{-\ell} \circ \widetilde{h}^{k}(\Gamma)$.

- Para $0 \leq n \leq n_{0}$, denotamos por $\widetilde{h}_{n}$ a aplicação $T^{-p_{n}} \circ \widetilde{h}^{-q_{n}}$.

- Dados três arcos essenciais simples $\Gamma^{\prime}, \Gamma^{\prime \prime}, \Gamma^{\prime \prime \prime}$, diremos que $\Gamma^{\prime}$ separa $\Gamma^{\prime \prime}$ e $\Gamma^{\prime \prime \prime}$, se eles forem disjuntos e satisfizerem $\Gamma^{\prime \prime}<\Gamma^{\prime}<\Gamma^{\prime \prime \prime}$ ou $\Gamma^{\prime \prime \prime}<\Gamma^{\prime}<$ $\Gamma^{\prime \prime}$.

Estamos prontos para demonstrar as duas afirmações mencionadas acima.

Afirmação 4.1 Para qualquer $n \in\left\{0, \ldots, n_{0}-1\right\}$, o arco $\Gamma$ separa os arcos $\Gamma\left(p_{n}, q_{n}\right)$ e $\Gamma\left(p_{n+1}, q_{n+1}\right)$. Ainda, se $n \neq 0$, então $\Gamma\left(p_{n+1}, q_{n+1}\right)$ separa $\Gamma e$ $\Gamma\left(p_{n-1}, q_{n-1}\right)$.

Demonstração. Esta prova será feita por indução decrescente com respeito a $n$. 
- Para $n=n_{0}-1$ :

Pela hipótese da Proposição 4.1, temos que $\Gamma\left(p^{\prime}, q^{\prime}\right)<\Gamma<\Gamma(p, q)$. Portanto, $\Gamma$ separa os $\operatorname{arcos} \Gamma\left(p_{n_{0}-1}, q_{n_{0}-1}\right)$ e $\Gamma\left(p_{n_{0}}, q_{n_{0}}\right)$.

- Para $n<n_{0}$ :

Suponhamos que o arco $\Gamma$ separa os $\operatorname{arcos} \Gamma\left(p_{n}, q_{n}\right)$ e $\Gamma\left(p_{n+1}, q_{n+1}\right)$. Sem perda de generalidade, assumamos

$$
\widetilde{h}_{n}(\Gamma)<\Gamma<\widetilde{h}_{n+1}(\Gamma) .
$$

Se $\widetilde{h}_{n}(\Gamma)<\Gamma$, pela Observação 3.1 , temos que $\Gamma<\widetilde{h}_{n}^{-a_{n+1}}$. Dado que $\widetilde{h}_{n+1}$ e $\widetilde{h}_{n}$ comutam (pois $T$ e $\widetilde{h}$ comutam), usando novamente o item (ii) da Observação 3.1, temos

$$
\widetilde{h}_{n+1}(\Gamma)<\widetilde{h}_{n}^{-a_{n+1}} \circ \widetilde{h}_{n+1}(\Gamma) .
$$

Agora, $\widetilde{h}_{n}^{-a_{n+1}} \circ \widetilde{h}_{n+1}=T^{a_{n+1} p_{n}-p_{n+1}} \circ \widetilde{h}^{-a_{n+1} q_{n}+q_{n+1}}$. Por (4.1), vemos que $\widetilde{h}_{n}^{-a_{n+1}} \circ \widetilde{h}_{n+1}=\widetilde{h}_{n-1}$. Portanto, juntando as desigualdades (4.2) e (4.3) obtemos finalmente

$\Gamma\left(p_{n}, q_{n}\right)=\widetilde{h}_{n}(\Gamma)<\Gamma<\widetilde{h}_{n+1}(\Gamma)=\Gamma\left(p_{n+1}, q_{n+1}\right)<\widetilde{h}_{n-1}(\Gamma)=\Gamma\left(p_{n-1}, q_{n-1}\right)$.

Similarmente, no caso

$$
\widetilde{h}_{n+1}(\Gamma)<\Gamma<\widetilde{h}_{n}(\Gamma)
$$

obtemos a desigualdade,

$$
\widetilde{h}_{n-1}(\Gamma)<\widetilde{h}_{n+1}(\Gamma)<\Gamma<\widetilde{h}_{n}(\Gamma) .
$$

Com isto, é fácil ver que a segunda conclusão desta afirmação também é verdadeira. 
Corolário 4.1 A ordem de toda a família dos arcos referentes à afirmação anterior é a seguinte (sem perda de generalidade, podemos supor que $n_{0}$ é par):

$$
\begin{gathered}
T^{-1}(\Gamma)=\Gamma\left(p_{0}, q_{0}\right)<\Gamma\left(p_{2}, q_{2}\right)<\ldots<\Gamma\left(p_{n_{0}}, q_{n_{0}}\right)<\Gamma, \\
\Gamma<\Gamma\left(p_{n_{0}-1}, q_{n_{0}-1}\right)<\ldots<\Gamma\left(p_{1}, q_{1}\right)<T(\Gamma) .
\end{gathered}
$$

Demonstração. Se $n_{0}$ é par e $\Gamma\left(p_{n_{0}}, q_{n_{0}}\right)<\Gamma<\Gamma\left(p_{n_{0}-1}, q_{n_{0}-1}\right)$, pela Afirmação 4.1, temos

$$
\Gamma\left(p_{n_{0}-2}, q_{n_{0}-2}\right)<\Gamma\left(p_{n_{0}}, q_{n_{0}}\right)<\Gamma<\Gamma\left(p_{n_{0}-1}, q_{n_{0}-1}\right)<\Gamma\left(p_{n_{0}-3}, q_{n_{0}-3}\right) .
$$

Desse modo, o resto das 'desigualdades' segue automaticamente aplicando uma e outra vez a Afirmação 4.1. A única desigualdade que não está contida nesta afirmação é a última, $\Gamma\left(p_{1}, q_{1}\right)<T(\Gamma)$. Mas, $\Gamma\left(p_{2}, q_{2}\right)<\Gamma$ implica que $T\left(\Gamma\left(p_{2}, q_{2}\right)\right)<T(\Gamma)$. Dado $T \circ T^{-p_{2}} \circ \widetilde{h}^{q_{2}}=T^{-p_{1}} \circ \widetilde{h}^{q_{1}}$ (lembre-se que $\frac{p_{1}}{q_{1}} \mathrm{e}$ $\frac{p_{2}}{q_{2}}=\frac{p_{1}}{q_{1}}+1$ são dois inteiros consecutivos) então $\Gamma\left(p_{1}, q_{1}\right)<T(\Gamma)$.

Afirmação 4.2 Para todo inteiro $n \in\left\{0, \ldots, n_{0}-1\right\}$, e todo par de inteiros $(\ell, k) \neq(0,0)$ com $\ell \in \mathbb{Z}$ e $k \in\left\{0, \ldots, q_{n}+q_{n+1}-1\right\}$, os arcos $\Gamma$ e $\Gamma(\ell, k)$ são disjuntos.

Demonstração. Para mostrar esta afirmação, provaremos primeiro o seguinte fato: para qualquer $n, \ell, k$ como os descritos nesta afirmação, o arco $\Gamma(\ell, k)$ não intercepta o disco topológico aberto cujo bordo em $\widetilde{\mathbb{A}}$ é $\Gamma\left(p_{n}, q_{n}\right) \cup$ $\Gamma\left(p_{n+1}, q_{n+1}\right)$. Isto, junto com a Afirmação 4.1, implicam de maneira automática esta afirmação. Nossa prova do fato anterior seguirá por indução.

- Para $n=0$ : 
Nesse caso, temos $k=0$, pois $q_{0}+q_{1}-1=0$. Pelo Corolário 4.1, sabemos

$$
T^{-1}(\Gamma)<\Gamma<\Gamma\left(p_{1}, q_{1}\right)<T(\Gamma) .
$$

Portanto, $\Gamma(\ell, 0) \leq T^{-1}(\Gamma)$ e $\Gamma\left(p_{1}, q_{1}\right)<\Gamma\left(-\ell^{\prime}, 0\right)$ para todo $\ell, \ell^{\prime} \in \mathbb{N}$.

- Para $(n-1) \geq 0$ :

Suponhamos que todo arco $\Gamma\left(\ell^{\prime}, k^{\prime}\right) \operatorname{com}\left(\ell^{\prime}, k^{\prime}\right) \neq(0,0), \ell^{\prime} \in \mathbb{Z}$ e $k^{\prime} \in$ $\left\{0, \ldots, q_{n-1}+q_{n}-1\right\}$ satisfaz $\Gamma\left(\ell^{\prime}, k^{\prime}\right) \leq \Gamma\left(p_{n}, q_{n}\right)$ ou $\Gamma\left(p_{n-1}, q_{n-1}\right) \leq \Gamma\left(\ell^{\prime}, k^{\prime}\right)$.

Observemos que, pela Afirmação 4.1, o arco $\Gamma$ separa $\Gamma\left(p_{n}, q_{n}\right)$ e $\Gamma\left(p_{n-1}, q_{n-1}\right)$. Sem perda de generalidade, assumamos

$$
\Gamma\left(p_{n}, q_{n}\right)<\Gamma<\Gamma\left(p_{n-1}, q_{n-1}\right) .
$$

Consideremos qualquer arco $\Gamma(\ell, k) \operatorname{com}(\ell, k) \neq(0,0), \ell \in \mathbb{Z}$ e $k \in$ $\left\{0, \ldots, q_{n}+q_{n+1}-1\right\}$. Da 'desigualdade' (4.4) junto com a Afirmação 4.1, obtém-se

$$
\Gamma\left(p_{n}, q_{n}\right)<\Gamma<\Gamma\left(p_{n+1}, q_{n+1}\right)<\Gamma\left(p_{n-1}, q_{n-1}\right) .
$$

Então, nosso objetivo será mostrar que $\Gamma(\ell, k) \leq \Gamma\left(p_{n}, q_{n}\right)$ ou $\Gamma\left(p_{n+1}, q_{n+1}\right)$ $\leq \Gamma(\ell, k)$. Se combinamos a 'desigualdade' (4.4) junto com a hipótese de indução, temos que o caso está feito para $k \leq q_{n-1}+q_{n}-1$.

Agora, suponhamos $k \geq q_{n-1}+q_{n}$. Dado $q_{n-1}+q_{n} \leq k \leq q_{n}+q_{n+1}-1=$ $a_{n+1} q_{n}+\left(q_{n-1}+q_{n}-1\right)$, podemos escrever $k=s q_{n}+k^{\prime} \operatorname{com} s \in\left\{1, \ldots, a_{n+1}\right\}$ e $k^{\prime} \in\left\{0, \ldots, q_{n-1}+q_{n}-1\right\}$. Similarmente, podemos escrever $\ell=s p_{n}+\ell^{\prime}$. Pela hipótese da indução, um dos seguintes três casos acontece:

- $\left(\ell^{\prime}, k^{\prime}\right)=(0,0)$ : Neste caso, teremos $\Gamma(\ell, k)=\Gamma\left(s p_{n}, s q_{n}\right)=\widetilde{h}_{n}^{s}(\Gamma) \leq$ $\widetilde{h}_{n}(\Gamma)=\Gamma\left(p_{n}, q_{n}\right)$. Isto segue pela aplicação da Observação 3.1 na desigualdade $\widetilde{h}_{n}(\Gamma)<\Gamma$. 
- $\Gamma\left(\ell^{\prime}, k^{\prime}\right)<\Gamma$ : Neste caso, $\Gamma(\ell, k)=\widetilde{h}_{n}^{s}\left(\Gamma\left(\ell^{\prime}, k^{\prime}\right)\right)<\widetilde{h}_{n}^{s}(\Gamma)$. Pelo caso anterior, concluímos que $\widetilde{h}_{n}^{s}\left(\Gamma\left(\ell^{\prime}, k^{\prime}\right)<\widetilde{h}_{n}(\Gamma)=\Gamma\left(p_{n}, q_{n}\right)\right.$.

- $\Gamma<\Gamma\left(\ell^{\prime}, k^{\prime}\right)$ : Neste caso, pela desigualdade (4.4) e pela hipótese de indução, $\Gamma\left(p_{n-1}, q_{n-1}\right) \leq \Gamma\left(\ell^{\prime}, k^{\prime}\right)$. Da desigualdade $\widetilde{h}_{n}(\Gamma)<\Gamma$ e $s \leq$ $a_{n+1}$ decorre

$$
\Gamma\left(p_{n+1}, q_{n+1}\right)=\widetilde{h}_{n-1}(\Gamma) \circ \widetilde{h}_{n}^{a_{n+1}}(\Gamma) \leq \widetilde{h}_{n-1} \circ \widetilde{h}_{n}^{s}(\Gamma)=\widetilde{h}_{n}^{s}\left(\Gamma\left(p_{n-1}, q_{n-1}\right)\right.
$$

Finalmente, pela desigualdade acima,

$$
\widetilde{h}_{n}^{s}\left(\Gamma\left(p_{n-1}, q_{n-1}\right) \leq \widetilde{h}_{n}^{s}\left(\Gamma\left(\ell^{\prime}, k^{\prime}\right)=\Gamma(\ell, k),\right.\right.
$$

o que completa a prova desta afirmação.

Prova da Proposição 4.1. Para mostrar o primeiro item, note que

$$
\Gamma(\ell, k) \cap \Gamma\left(\ell^{\prime}, k^{\prime}\right)=T^{-\ell^{\prime}} \circ \widetilde{h}^{-k^{\prime}}\left(\Gamma\left(\ell-\ell^{\prime}, k-k^{\prime}\right) \cap \Gamma\right) .
$$

Além disso, se $(\ell, k) \neq\left(\ell^{\prime}, k^{\prime}\right)$, então $\left(\ell-\ell^{\prime}, k-k^{\prime}\right) \neq(0,0)$. Da mesma forma, se $k, k^{\prime} \in\left\{0, \ldots, q+q^{\prime}-1\right\}$, então $k-k^{\prime} \in\left\{0, \ldots, q+q^{\prime}-1\right\}$ (podemos supor que $k \geq k^{\prime}$ ). Portanto, aplicando a afirmação anterior para $n=n_{0}-1$, vemos que $\Gamma\left(\ell-\ell^{\prime}, k-k^{\prime}\right) \cap \Gamma=\emptyset$. Isto encerra a prova do item (i).

Mostremos agora o segundo item. Primeiramente, é fácil ver

$$
\Gamma(\ell, k)<\Gamma\left(\ell^{\prime}, k^{\prime}\right) \Longleftrightarrow \Gamma\left(\ell-\ell^{\prime}, k-k^{\prime}\right)<\Gamma .
$$

Por outro lado, se $k \geq 0$ e $\alpha \in] \frac{p}{q}, \frac{p^{\prime}}{q^{\prime}}[$, é imediato que

$$
\begin{gathered}
k \frac{p^{\prime}}{q^{\prime}}-\ell \leq 0 \Rightarrow k \alpha-\ell<0, \\
k \frac{p}{q}-\ell \geq 0 \Rightarrow k \alpha-\ell>0 .
\end{gathered}
$$


Então, combinando isto, pode-se provar que, para qualquer $k \in\{0, \ldots, q+$ $\left.q^{\prime}-1\right\}$ e qualquer $\ell$,

$$
\begin{gathered}
T^{-\ell} \circ \widetilde{h}^{k}(\Gamma)<\Gamma \Rightarrow k \frac{p^{\prime}}{q^{\prime}}-\ell \leq 0, \\
\Gamma<T^{-\ell} \circ \widetilde{h}^{k}(\Gamma) \Rightarrow k \frac{p}{q}-\ell \geq 0 .
\end{gathered}
$$

Mostremos só o primeiro caso, pois para o segundo o raciocínio é similar.

Suponhamos que $T^{-\ell} \circ \widetilde{h}^{k}<\Gamma$. Dado $\Gamma<T^{-p} \circ \widetilde{h}^{-q}$, podemos dizer que $\frac{p}{q} \neq \frac{l}{k}$. Ainda, pelo item (iii) da Observação 3.1, temos

$$
\operatorname{Rot}\left(T^{-p} \circ \widetilde{h}^{-q}\right) \subset[0,+\infty[.
$$

Portanto, usando o Lema $2.2, \operatorname{Rot}(\widetilde{h}) \subset\left[\frac{p}{q},+\infty[\right.$.

Analogamente, pelo fato de $T^{-\ell} \circ \widetilde{h}^{k}<\Gamma$, o conjunto de rotação de $\widetilde{h}$ estará contido em $\left.]-\infty, \frac{\ell}{k}\right]$. Isto implica que $\frac{\ell}{k}-\frac{p}{q} \geq 0$, ou seja, $\frac{k \cdot p}{q}-\ell \leq 0$. Mas, pelo Lema 2.3 , o intervalo $] \frac{k \cdot p}{q}-\ell, \frac{k \cdot p^{\prime}}{q^{\prime}}-\ell[$ não contém inteiros, de modo que $\frac{k \cdot p^{\prime}}{q^{\prime}}-\ell \leq 0$.

Assim, para qualquer $k \in\left\{0, \ldots, q+q^{\prime}-1\right\}$, qualquer $\ell \in \mathbb{Z}$, e qualquer $\alpha \in] \frac{p}{q}, \frac{p^{\prime}}{q^{\prime}}[$

$$
\begin{aligned}
& T^{-\ell} \circ \widetilde{h}^{k}(\Gamma)<\Gamma \Rightarrow k \alpha-\ell<0, \\
& \Gamma<T^{-\ell} \circ \widetilde{h}^{k}(\Gamma) \Rightarrow k \alpha-\ell>0 .
\end{aligned}
$$

As contrapositivas destas duas implicações escrevem-se como,

$$
\begin{aligned}
& k \alpha-\ell>0 \Rightarrow \Gamma<T^{-\ell} \circ \widetilde{h}^{k}(\Gamma), \\
& k \alpha-\ell<0 \Rightarrow T^{-\ell} \circ \widetilde{h}^{k}(\Gamma)<\Gamma .
\end{aligned}
$$

Note que o caso $k \alpha-\ell=0$ não pode acontecer pois, pelo Lema 2.3, o intervalo $] \frac{k \cdot p}{q}, \frac{k \cdot p^{\prime}}{q^{\prime}}[$ não contém nenhum inteiro, isto é, $k \alpha-\ell$ nunca pode ser nulo. Por outro lado, se $k \alpha-\ell>0$, por exemplo, então $T^{-\ell} \circ \widetilde{h}^{k}(\Gamma) \cap \Gamma \neq \emptyset$ ou $\Gamma<T^{-\ell} \circ \widetilde{h}^{k}(\Gamma)$. Mas, anteriormente foi mostrado que $T^{-\ell} \circ \widetilde{h}^{k}(\Gamma) \cap \Gamma=\emptyset$. 
Daí, juntando estas quatro implicações, obtemos finalmente,

$$
\begin{aligned}
& T^{-\ell} \circ \widetilde{h}^{k}(\Gamma)<\Gamma \Longleftrightarrow k \alpha-\ell<0, \\
& \Gamma<T^{-\ell} \circ \widetilde{h}^{k}(\Gamma) \Longleftrightarrow k \alpha-\ell>0 .
\end{aligned}
$$

Isto completa a prova da proposição.

\subsection{Prova da Proposição 4.2}

Nesta seção começamos descrevendo o conceito das pseudo-órbitas, assim como várias conseqüências da sua definição. Posteriormente, daremos início à prova da Proposição 4.2 definindo a decomposição por tijolos e demonstrando algumas observações. Estes últimos fatos servirão de ajuda para poder simplificar a prova da proposição. Em toda esta seção, trabalharemos com as mesmas hipóteses da Proposição 4.2, isto é, dois homeomorfismos $\Phi_{1}, \Phi_{2}$ de $\widetilde{\mathbb{A}}$ que comutam entre si e com a translação $T$, tais que seus conjuntos de rotação estão contidos em $] 0,+\infty[$.

Definição 4.2 Uma seqüência $\left(x_{n}\right)_{n \geq 0}$ de pontos em $\widetilde{\mathbb{A}}$ chama-se uma $\left(\Phi_{1}, \Phi_{2}\right)$ órbita se para todo $n$, temos que $x_{n+1}=\Phi_{1}\left(x_{n}\right)$ ou $\Phi_{2}\left(x_{n}\right)$.

Denotemos por $d$ e \|\| a distância e norma Euclidianas, respectivamente, em $\widetilde{\mathbb{A}}=\mathbb{R} \times[0,1]$ e $\epsilon$ um número real positivo.

Definição 4.3 Uma $\epsilon-\left(\Phi_{1}, \Phi_{2}\right)$-pseudo-órbita é uma seqüência $\left(x_{n}\right)_{n \geq 0}$ de pontos em $\widetilde{\mathbb{A}}$ tal que, para todo $n$, temos que

$$
d\left(\Phi_{1}\left(x_{n}\right), x_{n+1}\right)<\epsilon \quad \text { ou } \quad d\left(\Phi_{2}\left(x_{n}\right), x_{n+1}\right)<\epsilon
$$


Além de sua definição ser simples, uma pseudo-órbita tem uma principal motivação, que é a seguinte: podemos escolher um $\epsilon>0$ de tal modo que, para qualquer $\epsilon-\left(\Phi_{1}, \Phi_{2}\right)$-pseudo-órbita, seu deslocamento esquerdo é universalmente limitado. Isto segue do seguinte resultado:

Proposição 4.3 Existem $\epsilon>0$ e $M>0$ tais que para qualquer $\epsilon-\left(\Phi_{1}, \Phi_{2}\right)$ pseudo-órbita $\left(x_{n}\right)_{n \geq 0}$ e para qualquer $n \geq 0$,

$$
p_{1}\left(x_{n}\right) \geq p_{1}\left(x_{0}\right)-M
$$

A prova central desta proposição segue das próximas duas afirmações, principalmente da segunda. Nesta segunda afirmação, limita-se o deslocamento esquerdo da $\epsilon-\left(\Phi_{1}, \Phi_{2}\right)$-pseudo-órbita para períodos grandes. Na primeira afirmação, este último fato é provado para $\left(\Phi_{1}, \Phi_{2}\right)$-órbitas.

Afirmação 4.3 Existe um inteiro $N>0$ com a seguinte propriedade. Para cada par de inteiros não negativos $\left(N_{1}, N_{2}\right)$ tais que $N_{1}+N_{2} \geq N$, e para todo ponto $x$ em $\widetilde{\mathbb{A}}$,

$$
p_{1}\left(\Phi_{1}^{N_{1}}\left(\Phi_{2}^{N_{2}}(x)\right)\right) \geq p_{1}(x)+2 .
$$

Em particular, se $\left(x_{n}\right)_{n \geq 0}$ é uma $\left(\Phi_{1}, \Phi_{2}\right)$-órbita, o termo $x_{N}$ é igual a $\Phi_{1}^{N_{1}}\left(\Phi_{2}^{N_{2}}\left(x_{0}\right)\right)$, onde $N_{1}+N_{2}=N$. Note que estamos usando o fato de que $\Phi_{1}$ e $\Phi_{2}$ comutam.

Demonstração. Aplicando o Lema 3.1, temos que existem números $\rho>$ $0, s \in \mathbb{R}$ tais que a desigualdade (3.1) se cumpre para os homeomorfismos $\Phi_{1}, \Phi_{2}$. Com efeito,

$$
p_{1}\left(\Phi_{1}^{n}(x)\right) \geq p_{1}(x)+\rho n-s \quad \text { e } \quad p_{1}\left(\Phi_{2}^{n}(x)\right) \geq p_{1}(x)+\rho n-s,
$$


para todo $x$ em $\widetilde{\mathbb{A}}$ e para todo inteiro positivo $n$.

Tomemos um par de inteiros não negativos $\left(N_{1}, N_{2}\right)$. Assim, podemos escrever $p_{1}\left(\Phi_{1}^{N_{1}}\left(\Phi_{2}^{N_{2}}(x)\right)\right)-p_{1}(x)$ como a soma,

$$
\left[p_{1}\left(\Phi_{1}^{N_{1}}\left(\Phi_{2}^{N_{2}}(x)\right)\right)-p_{1}\left(\Phi_{2}^{N_{2}}(x)\right)\right]+\left[p_{1}\left(\Phi_{2}^{N_{2}}(x)\right)-p_{1}(x)\right]
$$

Logo,

$$
p_{1}\left(\Phi_{1}^{N_{1}}\left(\Phi_{2}^{N_{2}}(x)\right)\right)-p_{1}(x) \geq \rho\left(N_{1}+N_{2}\right)-2 s .
$$

Com isto, para qualquer inteiro positivo $N$ tal que $\rho N-2 s \geq 2$ a conclusão do lema será verdadeira.

Vejamos agora o caso para as $\epsilon-\left(\Phi_{1}, \Phi_{2}\right)$-pseudo-órbitas.

Afirmação 4.4 Existe um inteiro $N>0$ e uma constante $\epsilon>0$ com a seguinte propriedade. Para toda $\epsilon-\left(\Phi_{1}, \Phi_{2}\right)$-pseudo-órbita $\left(x_{0}, \ldots, x_{N}\right)$ de tamanho $N$,

$$
p_{1}\left(x_{N}\right) \geq p_{1}\left(x_{0}\right)+1 \text {. }
$$

Demonstração. A idéia desta prova segue basicamente da 'continuidade' das $\left(\Phi_{1}, \Phi_{2}\right)$-órbitas. Em particular, seja $N$ o inteiro dado pela Afirmação 4.3. Dizemos que uma $\epsilon-\left(\Phi_{1}, \Phi_{2}\right)$-pseudo-órbita $\left(x_{0}, \ldots, x_{N}\right)$ de comprimento $N$ é de tipo $\sigma$, onde $\sigma \in\{1,2\}^{N}$, se para todo $n \in\{0, \ldots, N-1\}$ temos $d\left(x_{n+1}, \Phi_{\sigma_{n+1}}\left(x_{n}\right)\right)<\epsilon$. Dado que o conjunto $\{1,2\}^{N}$ é finito, vemos que é suficiente fazer a prova para cada tipo $\sigma$.

Fixemos o tipo $\sigma$ da pseudo-órbita. Dados um ponto $x_{0}$ em $\widetilde{\mathbb{A}}$ e uma seqüência de vetores do plano $\boldsymbol{v}=\left(\overrightarrow{v_{1}}, \ldots, \overrightarrow{v_{N}}\right)$, definimos recursivamente

$$
x_{1}:=\Phi_{\sigma_{1}}\left(x_{0}\right)+\overrightarrow{v_{1}}, \ldots, x_{N}:=\Phi_{\sigma_{N}}\left(x_{N-1}\right)+\overrightarrow{v_{N}}
$$


Identificaremos o espaço tangente $T_{x} \widetilde{\mathbb{A}}$ com o plano $\mathbb{R}^{2}$, e os vetores $\vec{v}_{i}$ serão escolhidos na bola unitária $\mathbb{D}$ do $\mathbb{R}^{2}$.

Agora, definimos a aplicação

$$
\begin{aligned}
\Im: \widetilde{\mathbb{A}} \times \mathbb{D}^{N} & \longrightarrow \mathbb{R}^{2}, \\
\left(x_{0}, \boldsymbol{v}\right) & \longrightarrow x_{N} .
\end{aligned}
$$

É claro ver que $\Im$ é contínua, pois

$$
x_{N}=\Phi_{\sigma_{N}}\left(\Phi_{\sigma_{N-1}}\left(\ldots\left(\Phi_{\sigma_{1}}\left(x_{0}\right)+\overrightarrow{v_{1}}\right) \ldots\right)+\overrightarrow{v_{N-1}}\right)+\overrightarrow{v_{N}}
$$

onde, $\Phi_{i}$ e a soma de vetores são aplicações contínuas. Além disso, pelo fato de que $\Phi_{i}$ comuta com a translação $T$, podemos notar que $\Im\left(T\left(x_{0}\right), \boldsymbol{v}\right)=$ $T\left(\Im\left(x_{0}, \boldsymbol{v}\right)\right)$. Desse modo, trabalharemos só no conjunto $[0,1] \times[0,1]$. Devido a compacidade deste último conjunto, podemos dizer que $\Im$ é uniformemente contínua. Portanto, existe um $\epsilon \in] 0,1\left[\right.$ tal que para todo $x_{0}$ em $\widetilde{\mathbb{A}}$ e para toda seqüência de vetores do plano $\boldsymbol{v}=\left(\overrightarrow{v_{1}}, \ldots, \overrightarrow{v_{N}}\right)$ cujas normas Euclidianas sejam menores que $\epsilon$, temos $d\left(\Im\left(x_{0}, \boldsymbol{v}\right), \Im\left(x_{0},(\overrightarrow{\mathbf{0}})\right)\right)<1$.

Neste ponto, pode-se notar o seguinte:

- Para toda $\epsilon-\left(\Phi_{1}, \Phi_{2}\right)$-pseudo-órbita $\left(x_{0}, \ldots, x_{N}\right)$ do comprimento $N$ de tipo $\sigma, x_{N}$ pode ser escrita como $\Im\left(x_{0}, \boldsymbol{v}\right)$ para alguma seqüência $\boldsymbol{v}=\left(\overrightarrow{v_{1}}, \ldots, \overrightarrow{v_{N}}\right) \operatorname{com}\left\|\overrightarrow{v_{i}}\right\|<\epsilon$.

- A igualdade $\Im\left(x_{0},(\overrightarrow{\mathbf{0}})\right)=\Phi_{\sigma_{N}} \circ \cdots \circ \Phi_{\sigma_{1}}\left(x_{0}\right)$.

De modo que, para toda $\epsilon-\left(\Phi_{1}, \Phi_{2}\right)$-pseudo-órbita $\left(x_{0}, \ldots, x_{N}\right)$ de comprimento $N$ e tipo $\sigma$, obtém-se

$$
p_{1}\left(x_{N}\right) \geq p_{1}\left(\Phi_{\sigma_{N}} \circ \cdots \circ \Phi_{\sigma_{1}}\left(x_{0}\right)\right)-1 .
$$


Aplicando a Afirmação 4.3 a $\left(\Phi_{1}, \Phi_{2}\right)$-órbita $\left(x_{0}, \Phi_{\sigma_{1}}\left(x_{0}\right), \ldots, \Phi_{\sigma_{N}} \circ \cdots \circ\right.$ $\left.\Phi_{\sigma_{1}}\left(x_{0}\right)\right)$ e usando o fato de que $\Phi_{1}$ e $\Phi_{2}$ comutam, tem-se $p_{1}\left(\Phi_{\sigma_{N}} \circ \cdots \circ \Phi_{\sigma_{1}}\left(x_{0}\right)\right)=p_{1}\left(\Phi_{1}^{N_{1}} \circ \Phi_{2}^{N_{2}}\left(x_{0}\right)\right) \geq p_{1}\left(x_{0}\right)+2, \quad$ onde $N_{1}+N_{2}=N$.

Somando estas duas desigualdades, concluímos finalmente que

$$
p_{1}\left(x_{N}\right) \geq p_{1}\left(x_{0}\right)+1 \text {. }
$$

Agora provaremos a Proposição 4.3.

Prova da Proposição 4.3. Sejam $N$ e $\epsilon>0$ o inteiro e a constante dados pela Afirmação 4.4. Então, para qualquer $\epsilon-\left(\Phi_{1}, \Phi_{2}\right)$-pseudo-órbita $\left(x_{0}, \ldots, x_{\ell}\right)$ de comprimento $\ell<N$, temos

$$
p_{1}\left(x_{\ell}\right) \geq p_{1}\left(\Phi_{i}\left(x_{\ell-1}\right)\right)-\epsilon, \quad \text { onde } i=1 \text { ou } 2 .
$$

Pelo Lema 3.1, obtém-se

$$
p_{1}\left(\Phi_{i}\left(x_{\ell-1}\right)\right) \geq p_{1}\left(x_{\ell-1}\right)+\rho-s \geq p_{1}\left(x_{\ell-1}\right)-s,
$$

pois $\rho$ é positivo, e $s$ é a cota dado pelo Lema 3.1. Somando estas duas desigualdades, chegamos a

$$
p_{1}\left(x_{\ell}\right) \geq p_{1}\left(x_{\ell-1}\right)-s-\epsilon .
$$

Aplicando novamente o Lema 3.1, temos

$$
p_{1}\left(x_{\ell-1}\right) \geq p_{1}\left(x_{\ell-2}\right)-s-\epsilon,
$$

portanto, 


$$
p_{1}\left(x_{\ell}\right) \geq p_{1}\left(x_{\ell-2}\right)-2 s-2 \epsilon .
$$

Assim, aplicando repetidamente o Lema 3.1, teremos

$$
p_{1}\left(x_{\ell}\right) \geq p_{1}\left(x_{0}\right)-\ell(s+\epsilon) \geq p_{1}\left(x_{0}\right)-N(s+\epsilon) .
$$

Consideremos agora qualquer inteiro $n$ e qualquer $\epsilon-\left(\Phi_{1}, \Phi_{2}\right)$-pseudoórbita $\left(x_{0}, \ldots, x_{n}\right)$ de comprimento $n$. Escrevamos $n$ como $k N+\ell$ onde $k \geq 0$ e $\ell \in\{0, \ldots, N-1\}$. Por um lado, da aplicação da Afirmação 4.4 na $\epsilon-$ $\left(\Phi_{1}, \Phi_{2}\right)$-pseudo-órbita $\left(x_{(k-1) N}, \ldots, x_{k N}\right)$ de comprimento $N$, resulta

$$
p_{1}\left(x_{k N}\right) \geq p_{1}\left(x_{(k-1) N}\right)+1,
$$

ou seja,

$$
\begin{aligned}
p_{1}\left(x_{N}\right) & \geq p_{1}\left(x_{0}\right)+1 \\
p_{1}\left(x_{2 N}\right) & \geq p_{1}\left(x_{N}\right)+1 \\
& \vdots \\
p_{1}\left(x_{k N}\right) & \geq p_{1}\left(x_{(k-1) N}\right)+1,
\end{aligned}
$$

de modo que,

$$
p_{1}\left(x_{k N}\right) \geq p_{1}\left(x_{0}\right)+k
$$

Por outra parte, pela desigualdade (4.5) aplicada na $\epsilon-\left(\Phi_{1}, \Phi_{2}\right)$-pseudoórbita $\left(x_{k N}, \ldots, x_{k N+\ell}\right)$ de comprimento $\ell$, segue que

$$
p_{1}\left(x_{n}\right) \geq p_{1}\left(x_{k N}\right)-N(s+\epsilon) .
$$

Daí, juntando as dois últimas desigualdades obtemos

$$
p_{1}\left(x_{n}\right)-p_{1}\left(x_{0}\right) \geq k-N(s+\epsilon) \geq-N(s+\epsilon) .
$$


Portanto, tomando $M=N(s+\epsilon)$ chegamos a desigualdade da Proposição 4.3 .

Com este resultado pronto, voltamos finalmente a prova da Proposição 4.2 .

Prova da Proposição 4.2. Primeiramente, construiremos o arco essencial simples. Para isto, consideremos uma decomposição por tijolos de $\mathbb{A}$, como mostra a Figura 4.1.

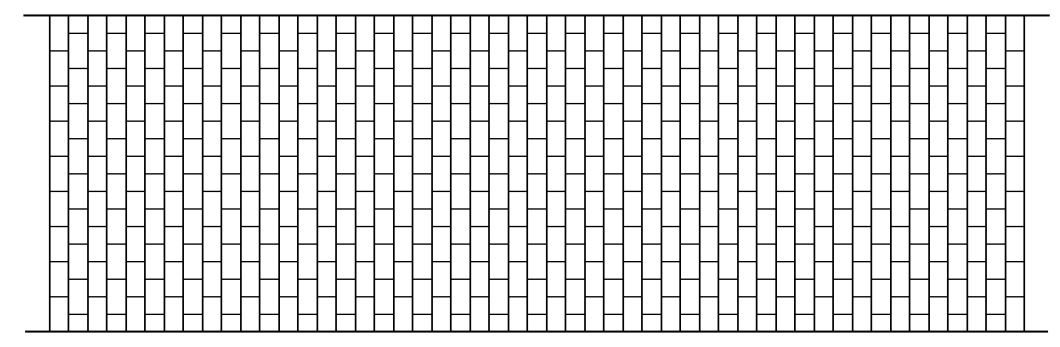

Figura 4.1: Decomposição por tijolos de $\mathbb{A}$

Basicamente, o que estamos fazendo é obter um gráfico triádico imerso, que denotaremos por $F$, em $\widetilde{\mathbb{A}}$ (triádico significa que cada vértice pertence exatamente a três arcos), tal que $F$ contenha as componentes da fronteira de $\widetilde{\mathbb{A}}$. Um tijolo é o fecho de um domínio complementar de $F$ de $\mathbb{A}$; isto é, um disco topológico fechado. Um requerimento importante para $F$ é o seguinte: todo tijolo tem que ter um diâmetro menor do que o número $\epsilon>0$ dado pela Proposição 4.3 (a métrica usada em $\mathbb{A}=\mathbb{S}^{1} \times[0,1]$ será a Euclidiana).

\section{Observação 4.1}

(i) Dado que F é triádico, a fronteira topológica da união de qualquer família de tijolos é uma 1-subvariedade em $\widetilde{\mathbb{A}}$, cuja fronteira está entre as componentes da fronteira de $\widetilde{\mathbb{A}}$. 
(ii) Qualquer subconjunto de $\widetilde{\mathbb{A}}$ está incluído no interior da união dos tijolos que ele intersecta (lembre-se que os tijolos são discos topológicos fechados).

Definição 4.4 Uma cadeia de tijolos (do tijolo $D_{0}$ até o tijolo $D_{i}$ ) é uma seqüência $\left(D_{0}, \ldots, D_{i}\right)$ de tijolos em $\widetilde{\mathbb{A}}$ tal que $\Phi_{1}\left(D_{0}\right) \cup \Phi_{2}\left(D_{0}\right)$ intersecta $D_{1}, \ldots, \Phi_{1}\left(D_{i-1}\right) \cup \Phi_{2}\left(D_{i-1}\right)$ intersecta $D_{i}$.

Consideremos $\Gamma_{0}=\{0\} \times[0,1]$; podemos supor que $\Gamma_{0}$ está incluído em $F$ (ver Figura 4.2). Definimos um subconjunto $A$ de $\widetilde{\mathbb{A}}$ da seguinte forma:

(a) Para qualquer tijolo $D_{0}$, denotemos por $\mathcal{D}\left(D_{0}\right)$ a união de todos os tijolos $D$ da decomposição tal que existe uma cadeia de tijolos de $D_{0}$ até $D$.

(b) O conjunto $A$ é a união de todos os conjuntos $\mathcal{D}\left(D_{0}\right)$, onde $D_{0}$ é tomado do conjunto sobre todos os tijolos que estão à direita do arco $\Gamma_{0}$ (o tijolo $D_{0}$ poderia intersectar $\Gamma_{0}$ ).

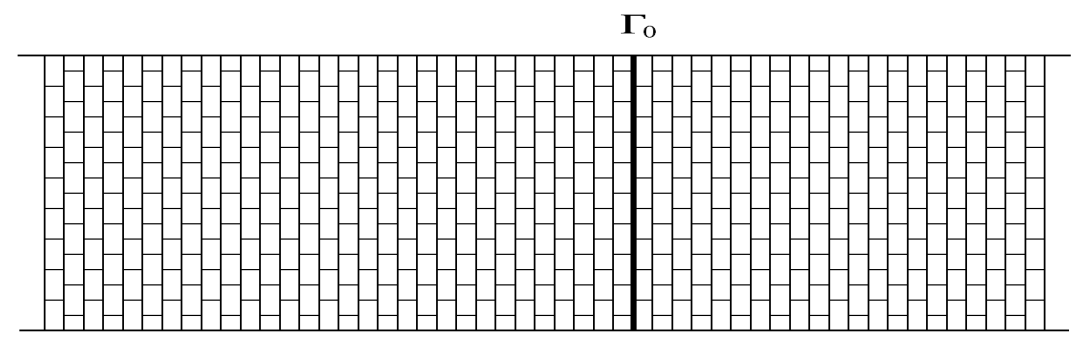

Figura 4.2: $\operatorname{Arco} \Gamma_{0}$ incluído em $F$

\section{Observação 4.2}

(i) Todos os tijolos que estão à direita de $\Gamma_{0}$ pertence ao conjunto A.

(ii) Existe uma constante $M>0$ tal que A está incluído em $[-M,+\infty[\times$ $[0,1]$. 
(iii) $O$ conjunto $A$ é um atrator estrito para $\Phi_{1}$ e $\Phi_{2}$, ou seja, $\Phi_{1}(A) \subset$ $\operatorname{Int}(A)$ e $\Phi_{2}(A) \subset \operatorname{Int}(A)$.

\section{Demonstração.}

(i) Basta considerar cadeias de um único tijolo.

(ii) Utilizando a Proposição 4.3, vemos que:

Sejam $\left(D_{o}, \ldots, D_{1}\right)$ uma cadeia de tijolos e $x$ qualquer ponto em $D_{i}$. Então, podemos construir facilmente uma $\epsilon-\left(\Phi_{1}, \Phi_{2}\right)$-pseudo-órbita $\left(x_{0}, \ldots, x_{i}\right)$ tal que $x_{0}$ está em $D_{0}$ e $x_{i}=x$. Em particular, seja $y_{i-1}$ qualquer ponto que pertence a $\left(\Phi_{1}\left(D_{i-1}\right) \cup \Phi_{2}\left(D_{i-1}\right)\right) \cap D_{i}$. Isto implica que existe um $x_{i-1}$ em $D_{i-1}$ tal que $y_{i-1}=\Phi_{1}\left(x_{i-1}\right)$ ou $\Phi_{2}\left(x_{i-1}\right)$ e $d\left(\Phi_{1}\left(x_{i-1}\right), x_{i}\right)<\epsilon$ ou $d\left(\Phi_{2}\left(x_{i-1}\right), x_{i}\right)<$ $\epsilon$. Analogamente, para $x_{i-1}$, conseguimos achar o elemento $x_{i-2}$ em $D_{i-2}$, e assim até encontrar o elemento $x_{0}$ em $D_{0}$ da $\epsilon-\left(\Phi_{1}, \Phi_{2}\right)$-pseudo-órbita.

Agora, lembremos que o $\epsilon>0$ foi dado pela Proposição 4.3, portanto, existe uma constante $M>0$, dada pela mesma proposição, tal que $p_{1}\left(x_{i}\right) \geq$ $p_{1}\left(x_{0}\right)-M$. Como $D_{0}$ está à direita de $\Gamma_{0}$, resulta que $p_{1}\left(x_{0}\right) \geq 0$, em consequiência, $D_{i}$ está contido em $[-M,+\infty[\times[0,1]$. Disto segue que $A$ está incluído em $[-M,+\infty[\times[0,1]$ (ver Figura 4.3).

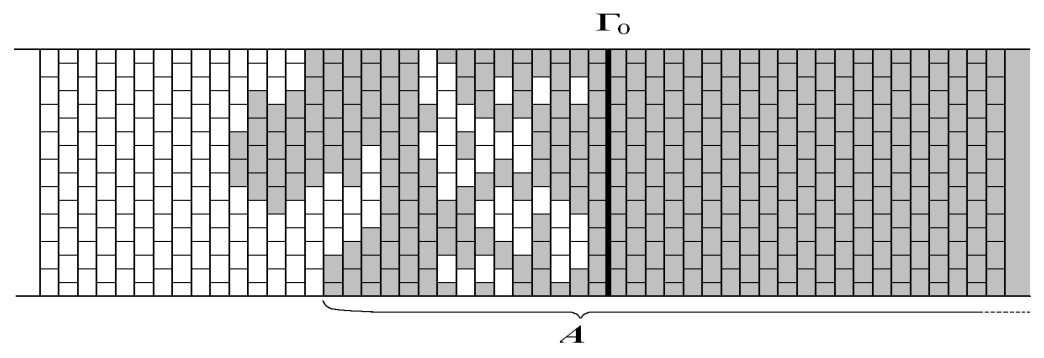

Figura 4.3: Conjunto $A$ 
(iii) Seja $D$ qualquer tijolo contido em $A$. Pela definição do conjunto $A$, existe uma cadeia de tijolos $\left(D_{0}, \ldots, D\right)$ onde $D_{0}$ está à direita de $\Gamma_{0}$. Então, para qualquer tijolo $D^{\prime}$ que intersecte $\Phi_{1}(D)$, temos que $\left(D_{0}, \ldots, D, D^{\prime}\right)$ vai ser também uma cadeia de tijolos. Portanto, $D^{\prime}$ pertence a $A$. Pelo item (ii) da Observação 4.1, sabemos que $\Phi_{1}(D)$ está incluído no interior da união dos tijolos que ele intersecta, de modo que $\Phi_{1}(D) \subset \operatorname{int}(A)$. Similar argumento serve para $\Phi_{2}$. Note a importância de haver definido os tijolos como conjuntos fechados para poder obter um atrator estrito.

Desse modo, definimos ao arco $\Gamma$ como a fronteira da componente conexa de $\widetilde{\mathbb{A}} \backslash A$ que contém $]-\infty, M[\times[0,1]$ (ver Figura 4.4). Pelo item (ii) da Observação 4.2, $\Gamma$ está bem definido. Ainda, pelo item (i) da Observação 4.1, temos que $\Gamma$ será um arco essencial simples. Portanto, se $\Phi_{1}(A) \subset$ $\operatorname{Int}(A)$ e $\Phi_{2}(A) \subset \operatorname{Int}(A)$, então $\Gamma$ é disjunto de suas imagens $\Phi_{1}(\Gamma)$ e $\Phi_{2}(\Gamma)$, tal que $\Gamma<\Phi_{1}(\Gamma)$ e $\Gamma<\Phi_{2}(\Gamma)$.

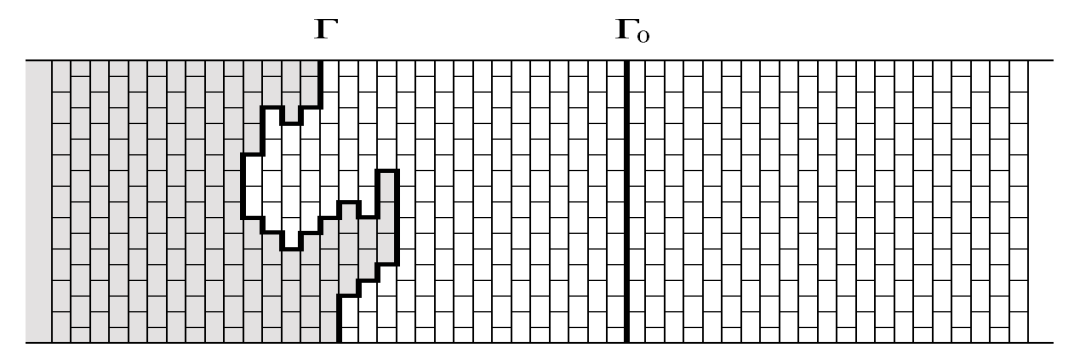

Figura 4.4: Arco essencial simples $\Gamma$

Isto termina a prova da Proposição 4.2. 


\section{Capítulo 5}

\section{O fecho da classe de conjugação de uma pseudo-rotação: Prova do Corolário 1.1}

Na introdução do Capítulo 1 apresentamos o seguinte resultado clássico dos homeomorfismos do círculo: Para qualquer homeomorfismo $h$ do círculo que preserva orientação e tem número de rotação $\alpha$, a rotação rígida de ângulo $\alpha$ está no fecho da classe de conjugação de $h$. Neste capítulo, faremos uma extensão deste resultado às pseudo-rotações irracionais do anel fechado, como o descreve o Corolário 1.1. De forma análoga ao caso do Teorema 1.1, este corolário será uma conseqüência imediata de outro resultado, no qual faremos uso, mais uma vez, da hipótese de que o conjunto de rotação está contido em um intervalo de Farey.

\subsection{Prova do Corolário 1.1}

Nesta seção provaremos o Corolário 1.1 fazendo uso da próxima proposição. 
Proposição 5.1 Seja $h: \mathbb{A} \rightarrow \mathbb{A}$ um homeomorfismo que é isotópico à identidade $e \widetilde{h}: \widetilde{\mathbb{A}} \rightarrow \widetilde{\mathbb{A}}$ um levantamento de $h$. Suponhamos que o conjunto de rotação Rot $(\widetilde{h})$ está contido em algum intervalo de Farey $] \frac{p}{q}, \frac{p^{\prime}}{q^{\prime}}[\subset \mathbb{R}$. Então, para qualquer número (racional ou irracional) $\alpha \in] \frac{p}{q}, \frac{p^{\prime}}{q^{\prime}}[$, existe um homeomorfismo $\sigma$ de $\mathbb{A}$, isotópico à identidade, tal que

$$
d\left(\sigma \circ h \circ \sigma^{-1}, R_{\alpha}\right)<\frac{40}{\min \left(q, q^{\prime}\right)} .
$$

Para provar o corolário, assumiremos a Proposição 5.1.

Prova do Corolário 1.2. Consideremos uma pseudo-rotação irracional $h$ de ângulo $\alpha$. Pelo Lema 2.4, $\alpha$ pertence a um intervalo de Farey $] \frac{p}{q}, \frac{p^{\prime}}{q^{\prime}}[$ com $q$ e $q^{\prime}$ arbitrariamente grandes. Portanto, pela Proposição 5.1, $h$ é conjugado a algum homeomorfismo $\sigma \circ h \circ \sigma^{-1}$ arbitrariamente perto da rotação rígida $R_{\alpha}$.

Isto termina a prova do Corolário 1.1

O resto deste capítulo terá como objetivo mostrar a Proposição 5.1.

\subsection{Preliminares: decomposição de homeo- morfismos do disco}

Nesta seção começamos mostrando que todo homeomorfismo $h$ que coincide com a identidade na fronteira do disco unitário fechado, pode ser decomposto como o produto de vários homeomorfismos, os quais estão arbitrariamente perto da identidade. Com isto, veremos que o mesmo resultado se realiza também quando o homeomorfismo é isotópico à identidade e coincide com ela só em dois arcos fechados disjuntos contidos na fronteira do disco. Este 
último fato, como veremos na próxima seção, ajudará a obter um resultado chave na demonstração da Proposição 5.1.

Antes de apresentar os dois resultados desta seção, é conveniente introduzir algumas notações e uma pequena definição. Usando-se a métrica Euclidiana de $\mathbb{R}^{2}$, denotemos por $\mathbb{D}$ ao disco unitário fechado. Denotemos também por $\mathrm{Homeo}^{+}(\mathbb{D})$ o conjunto dos homeomorfismos do disco $\mathbb{D}$ isotópicos à identidade (a definição de isotopia para o disco $\mathbb{D}$ é a mesma que foi definida no caso do anel $\mathbb{A}$ na Seção 2.3), e por $\operatorname{Homeo}(\mathbb{D}, \partial \mathbb{D})$ o conjunto de homeomorfismos que coincidem com a identidade na fronteira do $\mathbb{D}$. Nestos conjuntos, trabalharemos com a métrica usual $d\left(h, h^{\prime}\right)=\sup \left\{d\left(h(x), h^{\prime}(x)\right)\right.$ : $x \in \mathbb{D}\}$. Assim, dois homeomorfismos $h, h^{\prime}$ serão chamados de $\epsilon$-próximos se $d\left(h, h^{\prime}\right)<\epsilon$.

Observação 5.1 Seja h um homeomorfismo do disco $\mathbb{D}$. Então h é isotópico à identidade se e somente se

(i) $h$ preserva orientação, $e$

(ii) $h$ preserva a fronteira, ou seja, $h(\partial \mathbb{D})=\partial \mathbb{D}$.

Observação 5.2 Sejam $h, h^{\prime}$ dois elementos do conjunto Homeo(DD, $\left.\partial \mathbb{D}\right)$. Se $h, h^{\prime}$ são $\epsilon$-próximos, então o homeomorfismo $h \circ h^{\prime-1}$ é $\epsilon$-próximo da identidade. Em particular, se h é $\epsilon$-próximo da identidade, então o homeomorfismo $h^{-1}$ é também $\epsilon$-próximo da identidade.

Demonstração. De fato, se $d\left(h, h^{\prime}\right)<\epsilon$, então

$$
d\left(h(x), h^{\prime}(x)\right)<\epsilon \quad \forall x \in \mathbb{D} .
$$

Observemos que $h^{-1}, h^{-1}$ pertencem também ao conjunto Homeo( $(\mathbb{D}, \partial \mathbb{D})$. Logo, para todo $x \in \mathbb{D}$, existe um único ponto $y \in \mathbb{D}$, tal que $x=h^{-1}(y)$. Isso implica que 


$$
d\left(h \circ h^{\prime-1}(y), y\right)<\epsilon \quad \forall y \in \mathbb{D} \text {. }
$$

Com o mesmo argumento mostra-se a segunda conclusão desta observação.

O primeiro resultado desta seção é,

Lema 5.1 Para todo $\epsilon>0$, existe um $N \in \mathbb{N}$ tal que todo homeomorfismo $h \in \operatorname{Homeo}(\mathbb{D}, \partial \mathbb{D})$ pode ser escrito como a composta $h=h_{N} \circ \cdots \circ h_{1}$ de $N$ homeomorfismos em $\operatorname{Homeo}(\mathbb{D}, \partial \mathbb{D})$ os quais são $\epsilon$-próximos da identidade. Ainda, podemos escolher $N$ menor do que $\frac{4}{\epsilon}+4$.

Por outro lado, sejam $\alpha_{1}, \alpha_{2}$ dois arcos fechados contidos na fronteira $\partial \mathbb{D}$. Denotemos por $\mathrm{Homeo}^{+}\left(\mathbb{D}, \alpha_{1} \cup \alpha_{2}\right)$ o conjunto dos homeomorfismos do disco $\mathbb{D}$, isotópicos à identidade, que coincidem com a identidade em $\alpha_{1} \cup \alpha_{2}$. Note que quaisquer destes dois arcos poderia ser um só ponto.

Assim, um corolário importante do Lema 5.1 é o seguinte:

Corolário 5.1 Para todo $\epsilon>0$, existe um $N \in \mathbb{N}$ tal que todo homeomorfismo $h \in \mathrm{Homeo}^{+}\left(\mathbb{D}, \alpha_{1} \cup \alpha_{2}\right)$ pode ser escrito como o produto $h=h_{N} \circ \cdots \circ h_{1}$ de $N$ homeomorfismos em $\operatorname{Homeo}^{+}\left(\mathbb{D}, \alpha_{1} \cup \alpha_{2}\right)$ os quais são $\epsilon$-próximos da identidade. Ainda, podemos escolher $N$ menor do que $\left(\frac{4+2 \pi}{\epsilon}\right)+5$.

Passemos então a mostrar o Lema 5.1.

Prova do Lema 5.1. Comecemos fixando $0<\epsilon<\frac{1}{2}$, consideremos um homeomorfismo $h \in \operatorname{Homeo}(\mathbb{D}, \partial \mathbb{D})$.

Inicialmente, usando o lema de isotopia de Alexander, provaremos que $h$ pode ser escrito como o produto $h=h^{\prime} \circ h_{0}$ onde $h^{\prime}, h_{0}$ são homeomorfismos que pertencem ao conjunto $\operatorname{Homeo}(\mathbb{D}, \partial \mathbb{D})$, tais que $h_{0}$ é $\epsilon$-próximo da identidade e $h^{\prime}$ coincide com a identidade em uma vizinhança de $\partial \mathbb{D}$. 
Comecemos por estender $h$ a todo o plano $\mathbb{R}^{2}$, fazendo simplesmente com que ele coincida com a identidade em $\mathbb{R}^{2} \backslash \mathbb{D}$. Note que esta extensão está bem definida, pois $h$ coincide com a identidade na fronteira do disco $\mathbb{D}$. Agora, para $t \in] 0,1]$, consideremos o homeomorfismo

$$
\begin{aligned}
A_{t}: \mathbb{D} & \rightarrow \mathbb{D} \\
x & \mapsto t \cdot h\left(\frac{x}{t}\right) .
\end{aligned}
$$

Claramente, $A_{t} \in \operatorname{Homeo}(\mathbb{D}, \partial \mathbb{D})$, pois para qualquer $x \in \mathbb{D}$, se $\|x\| \geq t$, temos que $\left\|\frac{x}{t}\right\| \geq 1$, então $A_{t}(x)=t \cdot \frac{x}{t}=x$. Similarmente, se $\|x\|<t$ então $\left\|h\left(\frac{x}{t}\right)\right\|<1$, o que implica que $\left\|A_{t}(x)\right\|=t \cdot\left\|h\left(\frac{x}{t}\right)\right\|<t$. Isto mostra que $A_{t}$ coincide com a identidade fora da bola Euclidiana $B(\mathbf{0}, t)$ de centro $\mathbf{0} \mathrm{e}$ raio $t$. Ainda, pela definição do homeomorfismo $A_{t}$, a aplicação inversa dele é dada por $A_{t}^{-1}(x)=t \cdot h^{-1}\left(\frac{x}{t}\right)$ para todo $x \in \mathbb{D}$.

Definimos então $h^{\prime}:=A_{t_{0}}$ e $h_{0}:=A_{t_{0}}^{-1} \circ h$. É fácil ver que $h^{\prime}, h_{0} \in$ $\operatorname{Homeo}(\mathbb{D}, \partial \mathbb{D})$. Por outro lado, $h^{\prime}$ coincide com a identidade na vizinhança $\mathcal{V}=\left\{x \in \mathbb{D}:\|x\|>t_{0}\right\}$. Ainda, se $t_{0}$ está suficientemente perto de 1 , mostrase que $h_{0}$ satisfaz a propriedade requerida. Em particular, pela compacidade do disco unitário fechado $\mathbb{D}$, o homeomorfismo $A_{t_{0}}^{-1}$ é uniformemente contínuo. Portanto, para o $\epsilon$ fixado, temos que existe um $\delta>0$, tal que

$$
d(x, y)<\delta \Longrightarrow d\left(A_{t_{0}}^{-1}(x), A_{t_{0}}^{-1}(y)\right)<\frac{\epsilon}{2} .
$$

Para qualquer $x \in \mathbb{D}$, a distância Euclidiana $d\left(x, t_{0} x\right)=\left|1-t_{0}\right| \cdot\|x\| \leq$ $\left|1-t_{0}\right|$. Assim, para $\left|1-t_{0}\right|<\min \left(\delta, \frac{\epsilon}{2}\right)$, obtemos

$$
d\left(x, t_{0} x\right)<\delta
$$

o que implica que $d\left(A_{t_{0}}^{-1}(x), A_{t_{0}}^{-1}\left(t_{0} x\right)\right)<\frac{\epsilon}{2}$. Usando o fato de que $h$ é um homeomorfismo do disco $\mathbb{D}$, segue que existe um único $y \in \mathbb{D}$ tal que $x=h(y)$. Resulta então, 


$$
\begin{aligned}
d\left(A_{t_{0}}^{-1}(x), A_{t_{0}}^{-1}\left(t_{0} x\right)\right) & =d\left(t_{0} \cdot h^{-1}\left(\frac{x}{t_{0}}\right), t_{0} \cdot h^{-1}\left(\frac{t_{0} x}{t_{o}}\right)\right. \\
& =d\left(t_{0} \cdot h^{-1}\left(\frac{h(y)}{t_{0}}\right), t_{0} y\right) .
\end{aligned}
$$

Ou seja,

$$
d\left(t_{0} \cdot h^{-1}\left(\frac{h(y)}{t_{0}}\right), t_{0} y\right)<\frac{\epsilon}{2} .
$$

Analogamente, para o ponto $y$ obtido anteriormente,

$$
d\left(t_{0} y, y\right)=\left|1-t_{0}\right|<\frac{\epsilon}{2} .
$$

Somando estas duas últimas desigualdades, obtemos finalmente,

$$
d\left(t_{0} \cdot h^{-1}\left(\frac{h(y)}{t_{0}}\right), y\right)<\epsilon \quad \forall y \in \mathbb{D} .
$$

Resta só provar que o homeomorfismo $h^{\prime}$ pode ser escrito como o produto de $n$ elementos do conjunto $\operatorname{Homeo}(\mathbb{D}, \partial \mathbb{D})$ os quais sejam $\epsilon$-próximos da identidade, com $n<\frac{4}{\epsilon}+3$.

Tomemos o número $\mu=1-t_{0}$, onde $0<\mu<\frac{1}{2}$. Pela definição do homeomorfismo $A_{t_{0}}$, temos que $h^{\prime}$ coincide com a identidade fora da bola Euclidiana $B(\mathbf{0}, 1-\mu)$ de centro $\mathbf{0}$ e raio $1-\mu$. Definimos o seguinte homeomorfismo $\varphi$ do intervalo $[0,1]$ como (ver Figura 5.1)

$$
\varphi: t \longmapsto \begin{cases}\frac{t}{2}, & \text { se } 0 \leq t<\epsilon, \\ t-\frac{\epsilon}{2}, & \text { se } \epsilon \leq t<1-\mu, \\ \left(1+\frac{\epsilon}{2 \mu}\right) t-\frac{\epsilon}{2 \mu}, & \text { se } 1-\mu \leq t \leq 1\end{cases}
$$




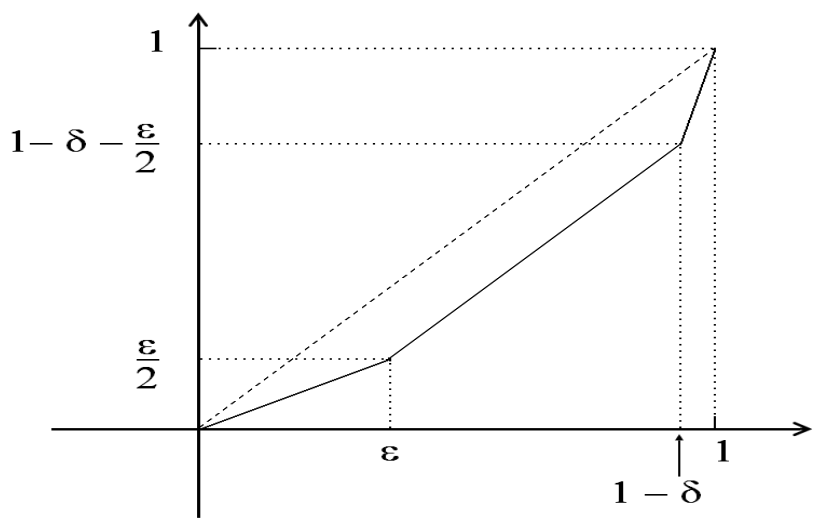

Figura 5.1: Homeomorfismo $\varphi$

Desse modo, consideremos agora o homeomorfismo radial $g_{\mu, \epsilon}$ no disco $\mathbb{D}$ definido por

$$
g_{\mu, \epsilon}: x \longmapsto \begin{cases}\varphi(\|x\|) \cdot \frac{x}{\|x\|}, & \text { se } x \neq \mathbf{0}, \\ \mathbf{0}, & \text { se } x=\mathbf{0} .\end{cases}
$$

A dinâmica do homeomorfismo $g_{\mu, \epsilon}$ é tomar qualquer ponto $x$ do disco $\mathbb{D}$ e projetá-lo radialmente na bola Euclidiana $B(\mathbf{0}, \varphi(\|x\|)$. Daí, o fato de $\varphi$ ser um homeomorfismo no intervalo $[0,1]$, implica que $g_{\mu, \epsilon}$ é um homeomorfismo do disco $\mathbb{D}$ que coincide com a identidade na fronteira do disco $\mathbb{D}$. Portanto, $g_{\mu, \epsilon} \in \operatorname{Homeo}(\mathbb{D}, \partial \mathbb{D})$, de modo que o homeomorfismo $g_{\mu, \epsilon}^{-1}$ também pertence ao conjunto $\operatorname{Homeo}(\mathbb{D}, \partial \mathbb{D})$.

Agora, verificaremos que $g_{\mu, \epsilon}$ é $\epsilon$-próximo da identidade. Observemos que para qualquer $x \in \mathbb{D}$,

$$
\begin{aligned}
d\left(g_{\mu, \epsilon}(x), x\right) & =\left\|\varphi(\|x\|) \cdot \frac{x}{\|x\|}-x\right\| \\
& =|\varphi(\|x\|)-\|x\|| .
\end{aligned}
$$

Por outro lado, 


$$
|\varphi(t)-t|= \begin{cases}\frac{t}{2}, & \text { se } 0 \leq t<\epsilon \\ \frac{\epsilon}{2}, & \text { se } \epsilon \leq t<1-\mu \\ \frac{\epsilon}{2 \mu}(1-t), & \text { se } 1-\mu \leq t \leq 1\end{cases}
$$

onde $|\varphi(t)-t| \leq \frac{\epsilon}{2}<\epsilon$, para qualquer $t \in[0,1]$. Isto implica que $d\left(g_{\mu, \epsilon}, \mathrm{id}\right)<$ $\epsilon$. Note que, pela Observação 5.2, o homeomorfismo $g_{\mu, \epsilon}^{-1}$ também será $\epsilon$-próximo da identidade.

Em seguida, estudemos a dinâmica do homeomorfismo $\varphi$ no ponto $1-\mu$.

$$
\begin{gathered}
\varphi(1-\mu)=(1-\mu)-\frac{\epsilon}{2}, \\
\varphi^{2}(1-\mu)=(1-\mu)-2 \cdot \frac{\epsilon}{2}, \\
\vdots \\
\varphi^{n}(1-\mu)=(1-\mu)-n \cdot \frac{\epsilon}{2},
\end{gathered}
$$

sempre que $\epsilon \leq \varphi^{n-1}(1-\mu)<1-\mu$, para qualquer inteiro positivo $n$. Assim, sem perda de generalidade, seja $n$ o primeiro inteiro positivo tal que $\varphi^{n-1}(1-\mu)<\epsilon$. Nesse caso, pela definição do homeomorfismo $\varphi$,

$$
\varphi\left(\varphi^{n-1}(1-\mu)\right)=\frac{\varphi^{n-1}(1-\mu)}{2}<\frac{\epsilon}{2},
$$

ou seja, $\varphi^{n}(1-\mu)<\frac{\epsilon}{2}$.

Mas,

$$
\varphi^{n-1}(1-\mu)=(1-\mu)-(n-1) \cdot \frac{\epsilon}{2}<\epsilon,
$$

o qual implica que $n>\frac{2}{\epsilon}\left(1-\mu-\frac{\epsilon}{2}\right)$. Logo, seja $m$ o inteiro em $\left[\frac{2}{\epsilon}, \frac{2}{\epsilon}+1[\right.$, pelo resultado anterior, segue que $m \geq n$ e por conseguinte $\varphi^{m}(1-\mu)<\frac{\epsilon}{2}$.

Analisemos agora a dinâmica do homeomorfismo $g_{\mu, \delta}$. Inicialmente, notemos que

$$
g_{\mu, \epsilon}^{m}(x)=\varphi^{m}(\|x\|) \cdot \frac{x}{\|x\|} \quad \text { e } \quad g_{\mu, \epsilon}^{m}(\mathbf{0})=\mathbf{0}
$$


Então, para todo $x \in B(\mathbf{0}, 1-\mu)$,

$$
\left\|g_{\mu, \epsilon}^{m}(x)\right\|=\varphi^{m}(\|x\|)=\varphi^{m}(1-\mu)<\frac{\epsilon}{2} .
$$

Em outras palavras, o homeomorfismo $g_{\mu, \epsilon}^{m}$ leva a bola $B(\mathbf{0}, 1-\mu)$ dentro da bola $B\left(\mathbf{0}, \frac{\epsilon}{2}\right)$. É imediato que para todo $x$ que está fora da bola $B\left(\mathbf{0}, \frac{\epsilon}{2}\right)$, o homeomorfismo $g_{\mu, \epsilon}^{-m}$ leva o ponto $x$ fora da bola $B(\mathbf{0}, 1-\mu)$, região do disco $\mathbb{D}$ onde o homeomorfismo $h^{\prime}$ coincide com a identidade. Neste caso,

$$
h^{\prime} \circ g_{\mu, \epsilon}^{-m}(x)=g_{\mu, \epsilon}^{-m}(x) .
$$

Definimos assim o homeomorfismo

$$
h_{b}:=g_{\mu, \epsilon}^{m} \circ h^{\prime} \circ g_{\mu, \epsilon}^{-m} .
$$

Pela observação anterior, o homeomorfismo $h_{b}$ coincide com a identidade fora da bola $B\left(\mathbf{0}, \frac{\epsilon}{2}\right)$. Isto implica que, para qualquer ponto $x$ que está dentro da bola $B\left(\mathbf{0}, \frac{\epsilon}{2}\right)$, sua imagem por $h_{b}$ tem que cair dentro da bola $B\left(\mathbf{0}, \frac{\epsilon}{2}\right)$ (caso contrário teríamos uma contradição) e portanto $d\left(x, h_{b}(x)\right)<\epsilon$. Podemos dizer então que o homeomorfismo $h_{b}$ é $\epsilon$-próximo da identidade.

Finalmente, escrevamos o homeomorfismo $h^{\prime}$ da seguinte forma,

$$
h^{\prime}=g_{\mu, \epsilon}^{-m} \circ h_{b} \circ g_{\mu, \epsilon}^{m} .
$$

Daí, concluímos que $h^{\prime}$ é o produto de $n=2 m+1$ elementos do conjunto $\operatorname{Homeo}(\mathbb{D}, \partial \mathbb{D})$, onde todos são $\epsilon$-próximos da identidade. Além disso, dado que o inteiro $m$ satisfaz $\frac{2}{\epsilon} \leq m<\frac{2}{\epsilon}+1$, teremos $n<\frac{4}{\epsilon}+3$.

Isto termina a prova do Lema 5.1.

Prova do Corolário 5.1. Antes de começar com a prova do Corolário 5.1, vamos descrever e mostrar algumas observações. 
Observação 5.3 Todo homeomorfismo crescente do intervalo [0,1] pode ser escrito como o produto de $N$ homeomorfismos que são $\epsilon$-próximos da identidade, com $N<\frac{1}{\epsilon}+1$.

Demonstração. Seja $h$ um homeomorfismo crescente de $[0,1]$. Se $d(h$,Id $)<$ $\epsilon$, o corolário será trivialmente certo com $N=1$. Assumiremos então que $d(h$, Id $) \geq \epsilon$. Portanto, existe um inteiro positivo $N>1$ tal que

$$
(N-1) \cdot \epsilon \leq d(h, I d)<N \cdot \epsilon .
$$

Consideremos o homeomorfismo

$$
h_{1}: x \longmapsto\left\{\begin{array}{lll}
x+\epsilon, & \text { se } & h(x)>x+\epsilon, \\
h(x), & \text { se } & x-\epsilon \leq h(x) \leq x+\epsilon, \\
x-\epsilon, & \text { se } & h(x)<x-\epsilon .
\end{array}\right.
$$

Logo, $d\left(h_{1}, \mathrm{Id}\right)=\epsilon$. Por outro lado, usando a definição de $h_{1}$,

$$
h(x)-h_{1}(x)= \begin{cases}(h(x)-x)-\epsilon, & \text { se } h(x)-x>\epsilon, \\ 0, & \text { se }-\epsilon \leq h(x)-x \leq \epsilon, \\ (x-h(x))-\epsilon, & \text { se } \epsilon<x-h(x) .\end{cases}
$$

Isto implica que

$$
d\left(h(x), h_{1}(x)\right)= \begin{cases}d(h(x), x)-\epsilon, & \text { se } d(h(x), x)>\epsilon, \\ 0 & \text { se } d(h(x), x) \leq \epsilon .\end{cases}
$$

Tomando o supremo das distâncias, obtemos que $d\left(h, h_{1}\right)=d(h$, Id $)-\epsilon$. Ainda, seguindo a mesma idéia feita na Observação 5.2 , temos que $d\left(h, h_{1}\right)=$ $d\left(h \circ h_{1}^{-1}, \mathrm{Id}\right)$, ou seja,

$$
d\left(h \circ h_{1}^{-1}, \mathrm{Id}\right)=d(h, \mathrm{Id})-\epsilon .
$$

A partir de (5.1), vale 


$$
(N-2) \cdot \epsilon \leq d\left(h \circ h_{1}^{-1}, \mathrm{Id}\right)<(N-1) \cdot \epsilon .
$$

Agora, o mesmo raciocínio aplicado ao homeomorfismo $h \circ h_{1}^{-1}$ conduz à seguinte desigualdade,

$$
(N-3) \cdot \epsilon \leq d\left(h \circ h_{1}^{-1} \circ h_{2}^{-1}, \mathrm{Id}\right)<(N-2) \cdot \epsilon,
$$

onde

$$
h_{2}: x \longmapsto \begin{cases}x+\epsilon, & \text { se } \quad\left(h \circ h_{1}^{-1}\right)(x)>x+\epsilon, \\ \left(h \circ h_{1}^{-1}\right)(x), & \text { se } \quad x-\epsilon \leq\left(h \circ h_{1}^{-1}\right)(x) \leq x+\epsilon, \\ x-\epsilon, & \text { se } \quad\left(h \circ h_{1}^{-1}\right)(x)<x-\epsilon\end{cases}
$$

Continuando do mesmo jeito chegamos ao homeomorfismo $h \circ h_{1}^{-1} \circ h_{2}^{-1} \circ$ $\cdots \circ h_{N-1}^{-1}$, tal que

$$
0 \leq d\left(h \circ h_{1}^{-1} \circ h_{2}^{-1} \circ \cdots \circ h_{N-1}^{-1}, \mathrm{Id}\right)<\epsilon .
$$

Assim, o homeomorfismo $g=h \circ h_{1}^{-1} \circ h_{2}^{-1} \circ \cdots \circ h_{N-1}^{-1}$ é $\epsilon$-próximo da identidade. Escrevendo,

$$
h=g \circ h_{N-1} \circ \cdots \circ h_{2} \circ h_{1}
$$

temos que $h$ é o produto de $N$ homeomorfismos $\epsilon$-próximos da identidade. Note que $N \leq \frac{d(h, \text { Id })}{\epsilon}+1$. Dado $d(h$, Id $)<1$, então $N<\frac{1}{\epsilon}+1$, como procurávamos.

Denotemos por $\mathrm{Homeo}^{+}\left(\partial \mathbb{D}, \alpha_{1} \cup \alpha_{2}\right)$ o espaço dos homeomorfismos do círculo $\partial \mathbb{D}$ que preservam a orientação e que coincidem com a identidade nos arcos fechados $\alpha_{1}$ e $\alpha_{2}$. O círculo $\partial \mathbb{D}$ será visto como a fronteira do disco unitário no plano Euclidiano; em lugar da métrica usual do círculo $\partial \mathbb{D}$, 
utilizaremos a métrica intrínseca do plano, isto é, a distância entre dois pontos $x, y \in \partial \mathbb{D}$ não será o comprimento do arco entre $x$ e $y$ senão o comprimento do segmento $[x y]$. Esta métrica induz uma métrica no espaço $\operatorname{Homeo}^{+}\left(\partial \mathbb{D}, \alpha_{1} \cup\right.$ $\left.\alpha_{2}\right)$, a qual denotaremos por $d_{\partial \mathbb{D}}$.

Observação 5.4 Todo elemento do conjunto $\mathrm{Homeo}^{+}\left(\partial \mathbb{D}, \alpha_{1} \cup \alpha_{2}\right)$ pode ser escrito como o produto de $N$ elementos em $\mathrm{Homeo}^{+}\left(\partial \mathbb{D}, \alpha_{1} \cup \alpha_{2}\right) \epsilon$-próximos da identidade, $\operatorname{com} N<\frac{2 \pi}{\epsilon}+1$.

Demonstração. Primeiramente, seja $\alpha$ qualquer arco fechado em $\partial \mathbb{D}$ cujos pontos extremais são $\left(\cos 2 \pi \beta_{1}, \sin 2 \pi \beta_{1}\right)$ e $\left(\cos 2 \pi \beta_{2}, \sin 2 \pi \beta_{2}\right)$. Usando a mesma notação da Seção 2.2 , suponhamos

$$
\left(\cos 2 \pi \beta_{1}, \sin 2 \pi \beta_{1}\right)<_{0}\left(\cos 2 \pi \beta_{2}, \sin 2 \pi \beta_{2}\right) .
$$

Assim, consideremos o homeomorfismo

$$
\begin{aligned}
\gamma:[0,1] & \rightarrow \alpha \\
t & \left.\mapsto\left(\cos \left(2 \pi(1-t) \beta_{1}+2 \pi t \beta_{2}\right), \sin \left(2 \pi(1-t) \beta_{1}+2 \pi t \beta_{2}\right)\right)\right)
\end{aligned}
$$

Para qualquer $t$ e $s$ no intervalo $[0,1]$ temos

$$
\begin{aligned}
d(\gamma(t), \gamma(s))^{2}= & {\left[\cos \left(2 \pi(1-t) \beta_{1}+2 \pi t \beta_{2}\right)-\cos \left(2 \pi(1-s) \beta_{1}+2 \pi s \beta_{2}\right)\right]^{2} } \\
& +\left[\sin \left(2 \pi(1-t) \beta_{1}+2 \pi t \beta_{2}\right)-\sin \left(2 \pi(1-s) \beta_{1}+2 \pi s \beta_{2}\right)\right]^{2}, \\
= & 2-2\left[\cos \left(\left(2 \pi(1-t) \beta_{1}+2 \pi t \beta_{2}\right)-\left(2 \pi(1-s) \beta_{1}+2 \pi s \beta_{2}\right)\right],\right. \\
\leq & 2-2\left[1-\frac{\left(2 \pi(1-t) \beta_{1}+2 \pi t \beta_{2}\right)-\left(2 \pi(1-s) \beta_{1}+2 \pi s \beta_{2}\right)^{2}}{2}\right] .
\end{aligned}
$$

Nesta última desigualdade, utilizamos o fato de que $\cos x \geq 1-\frac{x^{2}}{2}$. Assim, dado $\left|\beta_{1}-\beta_{2}\right| \leq 1$, então 


$$
d(\gamma(t), \gamma(s)) \leq 2 \pi d(t, s)
$$

ou seja, o homeomorfismo $\gamma$ tem constante de Lipschitz igual a $2 \pi$.

Seja então $f$ qualquer elemento do conjunto $\operatorname{Homeo}^{+}\left(\partial \mathbb{D}, \alpha_{1} \cup \alpha_{2}\right)$. Denotemos por $\alpha_{3}$ e $\alpha_{4}$ os dois arcos restantes do círculo $\partial \mathbb{D}$. Dado que $f$ é um homeomorfismo onde $\left.f\right|_{\alpha_{1} \cup \alpha_{2}}=\mathrm{id}$, temos que $f\left(\alpha_{3}\right)=\alpha_{3}$ e $f\left(\alpha_{4}\right)=\alpha_{4}$. Adicionalmente, podemos tomar os arcos $\alpha_{3}$ e $\alpha_{4}$ como arcos fechados, pois $f$ coincide com a identidade nos pontos extremais dos mesmos.

Consideremos assim o homeomorfismo $\left.\gamma^{-1} \circ f\right|_{\alpha_{3}} \circ \gamma$ do intervalo $[0,1]$, onde $\gamma:[0,1.] \rightarrow \alpha_{3}$. Pelo fato de $f$ preservar a orientação do círculo $\partial \mathbb{D}$, temos que $\left.\gamma^{-1} \circ f\right|_{\alpha_{3}} \circ \gamma$ é um homeomorfismo crescente. Logo, a Observação 5.3 garante que podemos escrever o homeomorfismo $\left.\gamma^{-1} \circ f\right|_{\alpha_{3}} \circ \gamma$ como produto de $N$ homeomorfismos que são $\frac{\epsilon}{2 \pi}$-próximos da identidade, com $N<\frac{2 \pi}{\epsilon}+1$. Em outras palavras,

$$
\left.\gamma^{-1} \circ f\right|_{\alpha_{3}} \circ \gamma=\gamma_{N} \circ \cdots \circ \gamma_{1}
$$

onde

$$
\gamma_{i}:[0,1] \rightarrow[0,1]
$$

$\mathrm{e}$

$$
d\left(\gamma_{i}, \mathrm{id}\right)<\frac{\epsilon}{2 \pi}, \quad 1 \leq i \leq N
$$

De (5.3) segue que o homeomorfismo $\left.f\right|_{\alpha_{3}}$ pode ser escrito como produto de $N$ homeomorfismos. Em particular:

$$
\left.f\right|_{\alpha_{3}}=\left(\gamma \circ \gamma_{N} \circ \gamma^{-1}\right) \circ \cdots \circ\left(\gamma \circ \gamma_{1} \circ \gamma^{-1}\right)
$$

onde 


$$
\gamma \circ \gamma_{i} \circ \gamma^{-1}: \alpha_{3} \rightarrow \alpha_{3}, \quad 1 \leq i \leq N
$$

Agora, fazendo uso da propriedade do homeomorfismo $\gamma$ ter constante de Lipschitz igual a $2 \pi$ vamos mostrar que $d_{\partial \mathbb{D}}\left(\gamma \circ \gamma_{i} \circ \gamma^{-1}\right.$,id $)<\epsilon$, para todo $1 \leq i \leq N$.

De fato, seja $x$ qualquer ponto do arco $\alpha_{3}$. Então $\gamma^{-1}(x) \in[0,1]$. Logo,

$$
d\left(\gamma_{i}\left(\gamma^{-1}(x)\right), \gamma^{-1}(x)\right)<\frac{\epsilon}{2 \pi} \quad 1 \leq i \leq N
$$

Utilizando (5.2) junto com esta última desigualdade, obtemos finalmente que, para todo $1 \leq i \leq N$,

$$
\begin{aligned}
d\left(\gamma\left(\gamma_{i}\left(\gamma^{-1}(x)\right)\right), \gamma\left(\gamma^{-1}(x)\right)\right) & \leq 2 \pi d\left(\gamma_{i}\left(\gamma^{-1}(x)\right), \gamma^{-1}(x)\right) \\
d\left(\gamma\left(\gamma_{i}\left(\gamma^{-1}(x)\right)\right), x\right) & <\epsilon .
\end{aligned}
$$

Daí, o homeomorfismo $\left.f\right|_{\alpha_{3}}$ pode ser escrito como o produto de $N$ homeomorfismos $\epsilon$-próximos da identidade, $\operatorname{com} N<\frac{2 \pi}{\epsilon}+1$.

Do mesmo jeito, pode-se chegar à mesma conclusão no caso do homeomorfismo $\left.f\right|_{\alpha_{4}}$ :

$$
\begin{aligned}
\left.f\right|_{\alpha_{4}} & =\left(\gamma \circ \gamma_{N}^{\prime} \circ \gamma^{-1}\right) \circ \cdots \circ\left(\gamma \circ \gamma_{1}^{\prime} \circ \gamma^{-1}\right), \\
d_{\partial \mathbb{D}}\left(\gamma \circ \gamma_{i}^{\prime} \circ \gamma^{-1}\right) & <\epsilon \quad 1 \leq i \leq N .
\end{aligned}
$$

Portanto,

$$
f=f_{N} \circ \cdots \circ f_{1}
$$

onde, para $1 \leq i \leq N$, o homeomorfismo $f_{i}$ do círculo $\partial \mathbb{D}$ está definido como, 


$$
f_{i}(x)= \begin{cases}\gamma \circ \gamma_{i} \circ \gamma^{-1}(x) & \text { se } x \in \alpha_{3}, \\ \gamma \circ \gamma_{i}^{\prime} \circ \gamma^{-1}(x) & \text { se } x \in \alpha_{4}, \\ x & \text { se } x \in \alpha_{1} \cup \alpha_{2}\end{cases}
$$

Dado que $\gamma \circ \gamma_{i} \circ \gamma^{-1}$ e $\gamma \circ \gamma_{i}^{\prime} \circ \gamma^{-1}$ coincidem com a identidade nos arcos $\alpha_{3}$ e $\alpha_{4}$, respectivamente, então $f_{i}$ está bem definido. Ainda, o homeomorfismo $f_{i}$ é um elemento de $\operatorname{Homeo}^{+}\left(\partial \mathbb{D}, \alpha_{1} \cup \alpha_{2}\right)$ e é $\epsilon$-próximo da identidade.

Voltemos agora à prova do Corolário 5.1. Fixemos um número $\epsilon>0 \mathrm{e}$ consideremos um homeomorfismo $h \in \operatorname{Homeo}^{+}\left(\mathbb{D}, \alpha_{1} \cup \alpha_{2}\right)$.

Dado que $h$ preserva a orientação, então $\left.h\right|_{\partial \mathbb{D}} \in \operatorname{Homeo}^{+}\left(\partial \mathbb{D}, \alpha_{1} \cup \alpha_{2}\right)$. De acordo com a Observação 5.4, temos

$$
\left.h\right|_{\partial \mathbb{D}}=H_{N_{1}} \circ \cdots \circ H_{1},
$$

onde $H_{i} \in \operatorname{Homeo}^{+}\left(\partial \mathbb{D}, \alpha_{1} \cup \alpha_{2}\right)$ e são $\epsilon$-próximos da identidade, com $N_{1}<$ $\frac{2 \pi}{\epsilon}+1$.

Agora, consideremos a seguinte 'aplicação de extensão circular' $\Phi$ (usando números complexos):

$$
\Phi:\left\{\begin{array}{ccc}
\left(\operatorname{Homeo}(\partial \mathbb{D}), d_{\partial \mathbb{D}}\right) & \rightarrow & (\operatorname{Homeo}(\mathbb{D}), d), \\
H & \mapsto\left(h: r e^{i \theta} \mapsto r H\left(e^{i \theta}\right) .\right.
\end{array}\right.
$$

Seja $h^{\prime}:=\Phi\left(\left.h\right|_{\partial \mathbb{D}}\right)$. De $(5.4)$ segue que $h^{\prime}=\Phi\left(H_{N_{1}} \circ \cdots \circ H_{1}\right)$.

É fácil verificar que $\Phi\left(H_{N_{1}} \circ \cdots \circ H_{1}\right)=\Phi\left(H_{N_{1}}\right) \circ \cdots \circ \Phi\left(H_{1}\right)$, ou seja

$$
h^{\prime}=\Phi\left(H_{N_{1}}\right) \circ \cdots \circ \Phi\left(H_{1}\right) .
$$

Em particular, para $1 \leq i \leq N_{1}$, o fato de $H_{i} \in \operatorname{Homeo}^{+}\left(\partial \mathbb{D}, \alpha_{1} \cup \alpha_{2}\right)$, implica que $\Phi\left(H_{i}\right) \in$ Homeo $^{+}\left(\mathbb{D}, \alpha_{1} \cup \alpha_{2}\right)$ (ver Observação 5.1), e portanto, $h^{\prime} \in \operatorname{Homeo}^{+}\left(\mathbb{D}, \alpha_{1} \cup \alpha_{2}\right)$, assim como sua inversa também pertence. Além 
disso, como $d_{\partial \mathbb{D}}\left(H_{i}\right.$,id $)<\epsilon$ então $d\left(\Phi\left(H_{i}\right)\right.$, id $)<\epsilon$ (lembre-se que em $\partial \mathbb{D}$ usamos a métrica extrínseca). Mais precisamente:

Seja $x=r e^{i \theta}$ qualquer ponto do disco $\mathbb{D}$. Então, para $1 \leq i \leq N_{1}$,

$$
\begin{aligned}
d\left(\left(\Phi\left(H_{i}\right)\right)\left(r e^{i \theta}\right), r e^{i \theta}\right) & =d\left(r H_{i}\left(e^{i \theta}\right), r e^{i \theta}\right) \\
& =r d\left(H_{i}\left(e^{i \theta}\right), e^{i \theta}\right) \\
& \leq d\left(H_{i}\left(e^{i \theta}\right), e^{i \theta}\right) \\
& <\epsilon .
\end{aligned}
$$

Por outro lado, aplicando a definição de $h^{\prime}$, obtemos que o homeomorfismo $h^{\prime^{-1}} \circ h$ é um elemento em Homeo $(\mathbb{D}, \partial \mathbb{D})$, sendo ele isotópico à identidade. Podemos então aplicar o Lema 5.1 ao homeomorfismo $h^{\prime^{-1}} \circ h$, e obter

$$
h^{\prime^{-1}} \circ h=h_{N_{2}} \circ \cdots \circ h_{1}
$$

onde $h_{i} \in \operatorname{Homeo}^{+}\left(\mathbb{D}, \alpha_{1} \cup \alpha_{2}\right)$ e são $\epsilon$-próximos da identidade, com $N_{2}<$ $\frac{4}{\epsilon}+4$.

Dessa maneira, juntando (5.5) e (5.6) finalizamos a prova do Corolário 5.1 escrevendo

$$
h=\Phi\left(H_{N_{1}}\right) \circ \cdots \circ \Phi\left(H_{1}\right) \circ\left(h_{N_{2}} \circ \cdots \circ h_{1}\right) .
$$

Nesta fórmula, $h$ é igual a produto de $\left(N_{1}+N_{2}\right)$ elementos de $\operatorname{Homeo}^{+}\left(\mathbb{D}, \alpha_{1} \cup\right.$ $\left.\alpha_{2}\right)$, que são $\epsilon$-próximos da identidade, com $\left(N_{1}+N_{2}\right)<\left(\frac{2 \pi+4}{\epsilon}\right)+5$.

\subsection{Prova da Proposição 5.1}

Nesta seção apresentamos a prova da Proposição 5.1, que para maior facilidade será dividida em três partes. Porém, antes de começar cada uma delas, vamos descrever uma certa família de subconjuntos do anel $\mathbb{A}$, assim 
como dois resultados chaves que eles satisfazem. O primeiro resultado está relacionado com esta família, e o segundo com um par de elementos dela.

Comecemos fixando um número $\alpha$ no intervalo $] \frac{p}{q}, \frac{p^{\prime}}{q^{\prime}}[$. Consideremos os arcos essenciais simples $\Gamma_{0}=\{0\} \times[0,1]$ em $\widetilde{\mathbb{A}}$, e $\gamma_{0}=\widetilde{\pi}\left(\Gamma_{0}\right)$ em $\mathbb{A}$. Similarmente, consideremos os discos topológicos fechados (ver Figura 5.2)

$\widetilde{D}:=R\left(\Gamma_{0}\right) \backslash \operatorname{Int}\left(R\left(T^{-p} \circ T_{\alpha}^{q}\left(\Gamma_{0}\right)\right)\right) \quad$ e $\quad \widetilde{D}^{\prime}:=R\left(T^{-p^{\prime}} \circ T_{\alpha}^{q^{\prime}}\left(\Gamma_{0}\right)\right) \backslash \operatorname{Int}\left(R\left(\Gamma_{0}\right)\right)$.

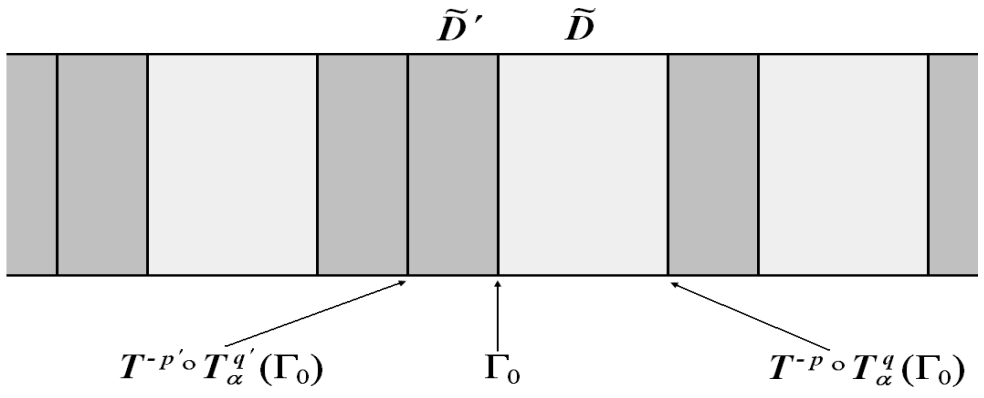

$\widetilde{\mathrm{A}}$

Figura 5.2: Dinâmica dos discos $\widetilde{D}$ e $\widetilde{D}^{\prime}$ em $\left.\widetilde{\mathbb{A}} \operatorname{com}\right] \frac{p}{q}, \frac{p^{\prime}}{q^{\prime}}[=] \frac{3}{5}, \frac{2}{3}[$

Chamemos $D=\widetilde{\pi}(\widetilde{D})$ e $D^{\prime}=\widetilde{\pi}\left(\widetilde{D}^{\prime}\right)$. Assim, fazendo uso da rotação $R_{\alpha}$, consideremos a família de discos topológicos fechados (ver Figura 5.3)

$$
\mathcal{D}=\left\{R_{\alpha}^{k}(D): k=0, \ldots, q^{\prime}-1\right\} \cup\left\{R_{\alpha}^{k^{\prime}}\left(D^{\prime}\right): k^{\prime}=0, \ldots, q-1\right\} .
$$




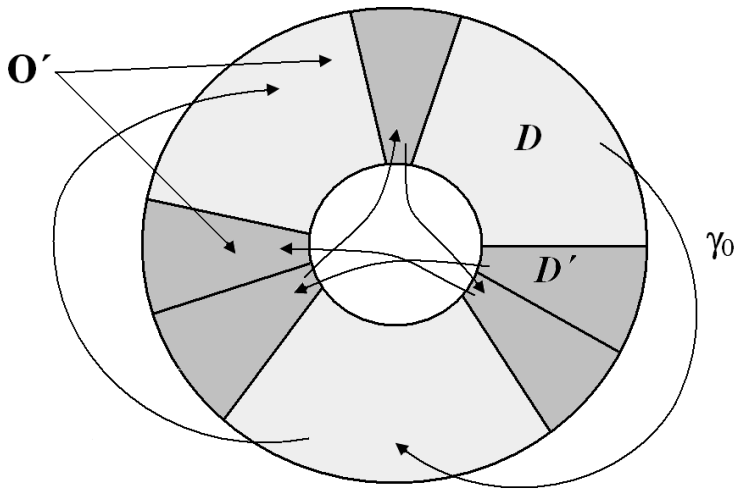

A

Figura 5.3: Dinâmica dos discos $D$ e $D^{\prime}$ em $\left.\mathbb{A} \operatorname{com}\right] \frac{p}{q}, \frac{p^{\prime}}{q^{\prime}}[=] \frac{3}{5}, \frac{2}{3}[$

O seguinte lema, que descreve duas propriedades desta família, é o primeiro de dois resultados importantes que serão demonstrados antes da Proposição 5.1 .

Lema 5.2 Seja $\mathcal{D}$ a família de discos descrita anteriormente. Então,

(i) os discos na família $\mathcal{D}$ cobrem o anel $\mathbb{A}$, e

(ii) seus interiores são dois a dois disjuntos.

Demonstração. Para mostrar a segunda propriedade é suficiente verificar que nenhum arco $R_{\alpha}^{k}\left(\gamma_{0}\right)$, com $k=\left\{0, \ldots, q+q^{\prime}-1\right\}$, intersecta o interior de $D$ ou $D^{\prime}$. Por exemplo, suponhamos que para um certo $k \in\left\{0, \ldots, q^{\prime}-1\right\}$ e $k^{\prime} \in\{0, \ldots, q-1\}$ temos que $\operatorname{Int}\left(R_{\alpha}^{k}(D) \cap R_{\alpha}^{k^{\prime}}\left(D^{\prime}\right)\right) \neq \emptyset$. Sem perda de generalidade, podemos supor que $k \geq k^{\prime}$. Então, $\operatorname{Int}\left(R_{\alpha}^{k-k^{\prime}}(D) \cap D^{\prime}\right) \neq \emptyset$, onde $k-k^{\prime} \in\left\{0, \ldots, q^{\prime}-1\right\}$. Conseqüentemente, um dos $\operatorname{arcos}, R_{\alpha}^{k-k^{\prime}}\left(\gamma_{0}\right)$ ou $R_{\alpha}^{q+k-k^{\prime}}\left(\gamma_{0}\right)$, intersectam o interior de $D^{\prime}$. Analogamente, pode-se verificar que a intersecção entre dois discos da família $\mathcal{D}$ implica que algum arco $R_{\alpha}^{k}\left(\gamma_{0}\right)$, com $k=\left\{0, \ldots, q+q^{\prime}-1\right\}$, intersecta o interior de $D$ ou $D^{\prime}$. 
Assim, no caso $k=0$, é óbvio que não é verdade, pois $\gamma_{0}$ pertence à fronteira dos dois discos. Agora, suponhamos, por absurdo, que existe algum arco $R_{\alpha}^{k}\left(\gamma_{0}\right)$, com $k \in\left\{1, \ldots, q+q^{\prime}-1\right\}$, que intersecta o interior de $D$ ou $D^{\prime}$. Por simplicidade, assumamos que ele intersecta $D^{\prime}$. Resulta então que existe um inteiro $n_{k}$ tal que

$$
q^{\prime} \alpha-p^{\prime}<k \alpha-n_{k}<0<q \alpha-p .
$$

Logo,

$$
\alpha<\frac{n_{k}}{k} .
$$

Como $\alpha \in] \frac{p}{q}, \frac{p^{\prime}}{q^{\prime}}\left[, \alpha<\frac{n_{k}}{k}<\frac{p^{\prime}}{q^{\prime}}\right.$ ou $\alpha<\frac{p^{\prime}}{q^{\prime}} \leq \frac{n_{k}}{k}$. O primeiro caso não pode acontecer. De fato, se $\alpha<\frac{n_{k}}{k}<\frac{p^{\prime}}{q^{\prime}}$, então $k \alpha<n_{k}<\frac{k \cdot p^{\prime}}{q^{\prime}}$. Mas, pelo Lema 2.3 , o intervalo $] \frac{k \cdot p}{q}, \frac{k \cdot p^{\prime}}{q^{\prime}}[$ não pode conter nenhum inteiro. Desse modo,

$$
\frac{p}{q}<\alpha<\frac{p^{\prime}}{q^{\prime}} \leq \frac{n_{k}}{k} .
$$

Agora, pelo fato de $\frac{p^{\prime}}{q^{\prime}}-\frac{p}{q}=\frac{1}{q q^{\prime}}$, temos que

$$
\left|q^{\prime} \alpha-p^{\prime}\right|=\frac{1}{q} \cdot \frac{\left|\alpha-\frac{p^{\prime}}{q^{\prime}}\right|}{\left(\frac{p^{\prime}}{q^{\prime}}-\frac{p}{q}\right)} .
$$

Similarmente,

$$
\left|k \alpha-n_{k}\right|=\frac{k \cdot\left|\alpha-\frac{n_{k}}{k}\right|}{\left(\frac{n_{k}}{k}-\frac{p}{q}\right)} \cdot\left(\frac{n_{k}}{k}-\frac{p}{q}\right) .
$$

Pela desigualdade (5.8), segue que $\frac{n_{k}}{k}-\frac{p}{q}=\frac{n_{k} q-k p}{k q}>0$. Como $n_{k} q-k p$ é um inteiro, isto implica

$$
\frac{n_{k}}{k}-\frac{p}{q} \geq \frac{1}{k q} .
$$


Desse modo, juntando (5.10) com (5.11), obtemos

$$
\left|k \alpha-n_{k}\right| \geq \frac{1}{q} \cdot \frac{\left|\alpha-\frac{n_{k}}{k}\right|}{\left(\frac{n_{k}}{k}-\frac{p}{q}\right)} .
$$

Por (5.7), temos que $\left|q^{\prime} \alpha-p^{\prime}\right|>\left|k \alpha-n_{k}\right|$. Usando (5.9) e (5.12) nesta desigualdade, obtemos finalmente

$$
\begin{aligned}
\frac{\left|\alpha-\frac{p^{\prime}}{q^{\prime}}\right|}{\left(\frac{p^{\prime}}{q^{\prime}}-\frac{p}{q}\right)} & >\frac{\left|\alpha-\frac{n_{k}}{k}\right|}{\left(\frac{n_{k}}{k}-\frac{p}{q}\right)} \\
& =\frac{\left(\frac{n_{k}}{k}-\frac{p^{\prime}}{q^{\prime}}\right)+\left(\frac{p^{\prime}}{q^{\prime}}-\alpha\right)}{\left(\frac{n_{k}}{k}-\frac{p^{\prime}}{q^{\prime}}\right)+\left(\frac{p^{\prime}}{q^{\prime}}-\frac{p}{q}\right)} .
\end{aligned}
$$

Assim, se $\frac{n_{k}}{k}-\frac{p \prime}{q^{\prime}}=0$ chegamos ao absurdo

$$
\frac{\left(\frac{p^{\prime}}{q^{\prime}}-\alpha\right)}{\left(\frac{p^{\prime}}{q^{\prime}}-\frac{p}{q}\right)}>\frac{\left(\frac{p^{\prime}}{q^{\prime}}-\alpha\right)}{\left(\frac{p^{\prime}}{q^{\prime}}-\frac{p}{q}\right)} .
$$

Por outro lado, se $\frac{n_{k}}{k}-\frac{p \prime}{q^{\prime}} \neq 0$ obtemos

$$
\left(\frac{p \prime}{q^{\prime}}-\alpha\right)>\left(\frac{p^{\prime}}{q^{\prime}}-\frac{p}{q}\right)
$$

o que também não pode acontecer.

O raciocínio é análogo quando o arco $R_{\alpha}^{k}\left(\gamma_{0}\right)$ intersecta o disco $D$.

Passemos agora a mostrar a primeira propriedade. Pelo Teorema 3.1, sabemos que os $\operatorname{arcos} R_{\alpha}^{k}\left(\gamma_{0}\right)$, com $k \in\left\{0, \ldots, q+q^{\prime}-1\right\}$, são dois a dois disjuntos. Portanto, se mostramos que cada um destes arcos está contido na fronteira de exatamente dois elementos da família $\mathcal{D}$, os quais pela segunda propriedade têm interiores disjuntos, então a família $\mathcal{D}$ cobrirá o anel $\mathbb{A}$.

Desse modo, sem perda de generalidade, suponhamos que $q^{\prime} \leq q$. Pela definição do disco $D$, vemos que os arcos que pertencem à fronteira dele, são $\gamma_{0}$ e $R_{\alpha}^{q}\left(\gamma_{0}\right)$. Então, os arcos que pertencem à fronteira do disco $R_{\alpha}^{\ell}(D)$, com $\ell \in\left\{0, \ldots, q^{\prime}-1\right\}$, são $R_{\alpha}^{\ell}\left(\gamma_{0}\right)$ e $R_{\alpha}^{q+\ell}\left(\gamma_{0}\right)$. Similarmente, os arcos que 
pertencem à fronteira do disco $R_{\alpha}^{\ell^{\prime}}\left(D^{\prime}\right)$, com $\ell^{\prime} \in\{0, \ldots, q-1\}$, são $R_{\alpha}^{\ell^{\prime}}\left(\gamma_{0}\right)$ e $R_{\alpha}^{q^{\prime}+\ell^{\prime}}\left(\gamma_{0}\right)$.

Por outro lado, fixemos um inteiro $k \in\left\{0, \ldots, q+q^{\prime}-1\right\}$. Então, dependendo dos possíveis valores que pode tomar o inteiro $k$, o arco $R_{\alpha}^{k}\left(\gamma_{0}\right)$ vai pertencer exatamente aos seguintes elementos da família $\mathcal{D}$ :

Se $k \in\left\{0, \ldots, q^{\prime}-1\right\} \Rightarrow R_{\alpha}^{k}\left(\gamma_{0}\right) \in R_{\alpha}^{k}(D)$ e $R_{\alpha}^{k}\left(D^{\prime}\right)$.

Se $k \in\left\{q^{\prime}, \ldots, q-1\right\} \Rightarrow R_{\alpha}^{k}\left(\gamma_{0}\right) \in R_{\alpha}^{k}(D)$ e $R_{\alpha}^{k-q^{\prime}}\left(D^{\prime}\right)$.

Se $k \in\left\{q, \ldots, q+q^{\prime}-1\right\} \Rightarrow R_{\alpha}^{k}\left(\gamma_{0}\right) \in R_{\alpha}^{k-q}(D)$ e $R_{\alpha}^{k-q^{\prime}}\left(D^{\prime}\right)$.

Isto termina a prova do Lema 5.2.

Desta maneira, denotemos por $O$ o disco topológico fechado $D^{\prime} \cup D$. A seguinte observação descreve algumas propriedades do disco $O$, que são consequiências direitas da definição dos discos $D$ e $D^{\prime}$.

\section{Observação 5.5}

(i) $O$ disco $O$ é igual a $R_{\alpha}^{q^{\prime}}(D) \cup R_{\alpha}^{q}\left(D^{\prime}\right)$.

(ii) A menos que $q=q^{\prime}=1, O$ é um disco topológico fechado.

(iii) $O$ interior de $O$ é disjunto de seus primeiros $\left(\min \left(q, q^{\prime}\right)-1\right)$ iterados por $R_{\alpha}^{-1}$.

(iv) A fronteira do disco $O$ (como uma variedade topológica) é uma curva simples fechada $C$, tal que

$$
C=R_{\alpha}^{q}\left(\gamma_{0}\right) \cup R_{\alpha}^{q^{\prime}}\left(\gamma_{0}\right) \cup C^{+} \cup C^{-},
$$

onde $C^{-}=\widetilde{\pi}\left(\left[q^{\prime} \alpha-p^{\prime}, q \alpha-p\right] \times\{0\}\right) e C^{+}=\widetilde{\pi}\left(\left[q^{\prime} \alpha-p^{\prime}, q \alpha-p\right] \times\{1\}\right)$. 
Note que pelo fato de usar a métrica Euclideina no anel $\mathbb{A}$, o disco $O$ é isométrico ao retângulo Euclidiano em $\mathbb{R}^{2}$, centrado em $(0,0)$, com largura $a=\left(q-q^{\prime}\right) \alpha-\left(p-p^{\prime}\right) \leq 1$ e altura $b=1$.

Assim, denotemos por $\operatorname{Homeo}^{+}\left(O, R_{\alpha}^{q}\left(\gamma_{0}\right) \cup R_{\alpha}^{q^{\prime}}\left(\gamma_{0}\right)\right)$ o espaço métrico dos homeomorfismos que preservam a orientação de $O$ os quais coincidem com a identidade em $R_{\alpha}^{q}\left(\gamma_{0}\right) \cup R_{\alpha}^{q^{\prime}}\left(\gamma_{0}\right)$. Apresentamos então o segundo utilizado na prova da Proposição 5.1.

Observação 5.6 Para todo $\epsilon>0$, existe um $N \in \mathbb{N}$ tal que todo homeomorfismo $h \in \operatorname{Homeo}^{+}\left(O, R_{\alpha}^{q}\left(\gamma_{0}\right) \cup R_{\alpha}^{q^{\prime}}\left(\gamma_{0}\right)\right)$ pode ser escrito como o produto $h=h_{N} \circ \cdots \circ h_{1}$ de $N$ homeomorfismos em $\operatorname{Homeo}^{+}\left(O, R_{\alpha}^{q}\left(\gamma_{0}\right) \cup R_{\alpha}^{q^{\prime}}\left(\gamma_{0}\right)\right)$ os quais são $\epsilon$-próximos da identidade. Ainda, podemos escolher $N$ menor do que $2\left(\frac{4+2 \pi}{\epsilon}\right)+5$.

Demonstração. De fato, a observação acima é uma conseqüência direta do Corolário 5.1. Para isso, introduzimos em primeiro lugar o homeomorfismo $\psi: \mathbb{D} \rightarrow O$ definido por

$$
\psi:(x, y) \longmapsto \begin{cases}\frac{\sqrt{x^{2}+y^{2}}}{\max (|x|,|y|)} \cdot\left(\frac{a \cdot x}{2}, \frac{b \cdot y}{2}\right), & \text { se }(x, y) \neq(0,0), \\ (0,0), & \text { se }(x, y)=(0,0) .\end{cases}
$$

Denotando por $d_{O}$ e $d_{\mathbb{D}}$ a métrica Euclidiana no disco $O$ e $\mathbb{D}$, respectivamente, obtemos a seguinte estimativa:

Sejam $z=(x, y)$ e $z^{\prime}=\left(x^{\prime}, y^{\prime}\right)$ dois pontos do disco $\mathbb{D}$, então

$$
d_{O}\left(\psi(z), \psi\left(z^{\prime}\right)\right) \leq 2 d_{\mathbb{D}}\left(z, z^{\prime}\right) .
$$

Em particular, chamemos

$$
c=\frac{\sqrt{x^{2}+y^{2}}}{\max (|x|,|y|)} \quad \text { e } \quad c^{\prime}=\frac{\sqrt{x^{\prime 2}+y^{\prime 2}}}{\max \left(\left|x^{\prime}\right|,\left|y^{\prime}\right|\right)} .
$$


Como $\sqrt{x^{2}+y^{2}} \leq 2 \cdot \max (|x|,|y|)$ para todo $(x, y)$ no $\mathbb{R}^{2}$, vemos que

$$
\frac{a \cdot c}{2}, \frac{a \cdot c^{\prime}}{2} \leq 1 \quad \text { e } \quad \frac{b \cdot c}{2}, \frac{b \cdot c^{\prime}}{2} \leq 1
$$

Agora,

$$
\begin{aligned}
4 d_{\mathbb{D}}^{2}\left(z, z^{\prime}\right)-d_{O}^{2}\left(\psi(z), \psi\left(z^{\prime}\right)\right)= & 4\left(x-x^{\prime}\right)^{2}-\left(\frac{a \cdot c \cdot x}{2}-\frac{a \cdot c^{\prime} \cdot x^{\prime}}{2}\right)^{2}+ \\
& 4\left(y-y^{\prime}\right)^{2}-\left(\frac{a \cdot b \cdot y}{2}-\frac{a \cdot b^{\prime} \cdot y^{\prime}}{2}\right)^{2}
\end{aligned}
$$

Por outro lado,

$$
\begin{aligned}
4\left(x-x^{\prime}\right)^{2}-\left(\frac{a \cdot c \cdot x}{2}-\frac{a \cdot c^{\prime} \cdot x^{\prime}}{2}\right)^{2}= & {\left[2\left(x-x^{\prime}\right)+\left(\frac{a \cdot c \cdot x}{2}-\frac{a \cdot c^{\prime} \cdot x^{\prime}}{2}\right)\right] . } \\
& {\left[2\left(x-x^{\prime}\right)-\left(\frac{a \cdot c \cdot x}{2}-\frac{a \cdot c^{\prime} \cdot x^{\prime}}{2}\right)\right], } \\
= & {\left.\left[\left(2+\frac{a \cdot c}{2}\right) x-\left(2+\frac{a \cdot c^{\prime}}{2}\right) x^{\prime}\right)\right] . } \\
& {\left.\left[\left(2-\frac{a \cdot c}{2}\right) x-\left(2-\frac{a \cdot c^{\prime}}{2}\right) x^{\prime}\right)\right], } \\
= & {\left[\sqrt{\left(2+\frac{a \cdot c}{2}\right)\left(2-\frac{a \cdot c^{\prime}}{2}\right) x-}\right.} \\
\geq & \left.\sqrt{\left(2+\frac{a \cdot c}{2}\right)\left(2-\frac{a \cdot c^{\prime}}{2}\right)} x^{\prime}\right]^{2} \\
\geq &
\end{aligned}
$$

Observemos que pelas desigualdades (5.14), as raízes quadradas obtidas acima são sempre reais, o que assegura que $4\left(x-x^{\prime}\right)^{2}-\left(\frac{a \cdot c \cdot x}{2}-\frac{a \cdot c^{\prime} \cdot x^{\prime}}{2}\right)^{2}$ seja maior ou igual a zero. De maneira similar pode-se obter $4\left(y-y^{\prime}\right)^{2}-\left(\frac{a \cdot b \cdot y}{2}-\right.$ $\left.\frac{a \cdot b^{\prime} y^{\prime}}{2}\right)^{2} \geq 0$. Isto implica que $4 d_{\mathbb{D}}^{2}\left(z, z^{\prime}\right)-d_{O}^{2}\left(\psi(z), \psi\left(z^{\prime}\right)\right) \geq 0$. Em outras palavras, $d_{O}\left(\psi(z), \psi\left(z^{\prime}\right)\right) \leq 2 \cdot d_{\mathbb{D}}\left(z, z^{\prime}\right)$.

Consideremos um homeomorfismo $h \in \operatorname{Homeo}^{+}\left(O, R_{\alpha}^{q}\left(\gamma_{0}\right) \cup R_{\alpha}^{q^{\prime}}\left(\gamma_{0}\right)\right.$. Portanto, $\psi^{-1} \circ h \circ \psi \in$ Homeo $^{+}\left(\mathbb{D}, \psi^{-1}\left(R_{\alpha}^{q}\left(\gamma_{0}\right) \cup R_{\alpha}^{q^{\prime}}\left(\gamma_{0}\right)\right)\right)$. Pelo Corolário 5.1, temos

$$
\psi^{-1} \circ h \circ \psi=h_{N} \circ \cdots \circ h_{1}
$$

onde, para $1 \leq i \leq N$, 


$$
h_{i} \in \operatorname{Homeo}^{+}\left(\mathbb{D}, \psi^{-1}\left(R_{\alpha}^{q}\left(\gamma_{0}\right) \cup R_{\alpha}^{q^{\prime}}\left(\gamma_{0}\right)\right)\right) \text {, }
$$

e $d_{\mathbb{D}}\left(h_{i}\right.$, id $)<\frac{\epsilon}{2}$. Ainda, podemos escolher $N<2\left(\frac{4+2 \pi}{\epsilon}\right)+5$.

Desse modo, $h$ pode ser escrito como

$$
h=\left(\psi \circ h_{N} \circ \psi^{-1}\right) \cdots \circ\left(\psi \circ h_{1} \circ \psi^{-1}\right),
$$

onde $\left.\left(\psi \circ h_{i} \circ \psi^{-1}\right) \in \operatorname{Homeo}^{+}\left(O, R_{\alpha}^{q}\left(\gamma_{0}\right) \cup R_{\alpha}^{q^{\prime}}\left(\gamma_{0}\right)\right)\right)$.

Finalmente, seja $w$ qualquer ponto do disco $O$. Então, para $1 \leq i \leq N$,

$$
d_{\mathbb{D}}\left(h_{i}\left(\psi^{-1}(w)\right), \psi^{-1}(w)\right)<\frac{\epsilon}{2} .
$$

Pela estimativa (5.13), obtemos

$$
d_{O}\left(\psi\left(h_{i}\left(\psi^{-1}(w)\right)\right), w\right) \leq 2 d_{\mathbb{D}}\left(h_{i}\left(\psi^{-1}(w)\right), \psi^{-1}(w)\right)<\epsilon
$$

ou seja,

$$
d_{O}\left(\psi \circ h_{i} \circ \psi^{-1}, i d\right)<\epsilon .
$$

Isto conclui a prova da Observação 5.6.

Cabe notar que esta observação também é verdadeira se mudamos o disco $O$ pelo disco $O^{\prime}=R_{\alpha}^{-1}(O)$, pois $R_{\alpha}^{-1}$ é uma isometria.

Estamos prontos para demonstrar a Proposição 5.1.

\subsubsection{Primeira parte da prova}

Considerando o homeomorfismo $h$ da hipótese da Proposição 5.1, construiremos nesta primeira parte, um homeomorfismo $\sigma_{a}: \mathbb{A} \rightarrow \mathbb{A}$, isotópico à identidade, tal que o homeomorfismo $h_{a}=\sigma_{a} \circ h \circ \sigma_{a}^{-1}$ coincida com a rotação $R_{\alpha}$ em cada arco $R_{\alpha}^{k}\left(\gamma_{0}\right) \operatorname{com} k \in\left\{0, \ldots, q+q^{\prime}-2\right\}$. 
Aplicando o Teorema 3.1 (Teorema do 'arco de translação') ao homeomorfismo $h$, temos que existe um arco essencial simples $\gamma$ em $\mathbb{A}$ tal que os $\operatorname{arcos} \gamma, \ldots, h^{q+q^{\prime}-1}(\gamma)$ são dois a dois disjuntos.

Denotando por $\gamma_{0}$ o arco $\{0\} \times[0,1]$ em $\mathbb{A}$, consideremos o homeomorfismo

$$
\sigma_{a}: \gamma \rightarrow \gamma_{0}
$$

que leva o ponto extremo de $\gamma$ que está em $\mathbb{S}^{1} \times\{1\}$ (resp. em $\left.\mathbb{S}^{1} \times\{0\}\right)$ no ponto extremo de $\gamma_{0}$ que está em $\mathbb{S}^{1} \times\{1\}$ (resp. em $\mathbb{S}^{1} \times\{0\}$ ). Pelo fato de que os $\operatorname{arcos} \gamma, \ldots, h^{q+q^{\prime}-1}(\gamma)$ são dois a dois disjuntos, podemos extender o homeomorfismo $\sigma_{a}$ à união destes arcos. Em particular, seja o homeomorfismo

$$
\begin{aligned}
\sigma_{a}: \bigcup_{k=0}^{q+q^{\prime}-1} h^{k}(\gamma) & \longrightarrow \bigcup_{k=0}^{q+q^{\prime}-1} R_{\alpha}^{k}\left(\gamma_{0}\right), \\
x \in h^{k}(\gamma) & \longmapsto\left(\left.R_{\alpha}^{k} \circ \sigma_{a}\right|_{\gamma} \circ h^{-k}\right)(x) .
\end{aligned}
$$

Note que o homeomorfismo $\sigma_{a}$ não só leva o $\operatorname{arco} h^{k}(\gamma)$ ao $\operatorname{arco} R_{\alpha}^{k}\left(\gamma_{0}\right)$, como também leva o ponto extremo de $h^{k}(\gamma)$ que está em $\mathbb{S}^{1} \times\{1\}$ (resp. em $\mathbb{S}^{1} \times\{0\}$ ) ao ponto extremo de $R_{\alpha}^{k}\left(\gamma_{0}\right)$ que está em $\mathbb{S}^{1} \times\{1\}$ (resp. em $\left.\mathbb{S}^{1} \times\{0\}\right)$.

O próximo passo será estender o homeomorfismo $\sigma_{a}$ ao anel $\mathbb{A}$. Tal extensão usa fortemente o seguinte resultado conhecido como o Teorema de Jordan-Schoenflies:

Teorema 5.3.1 Uma curva fechada simples $\Omega$ no plano $\mathbb{R}^{2}$ o separa em duas regiões. Ainda, existe um homeomorfismo $\Phi: \mathbb{R}^{2} \rightarrow \mathbb{R}^{2}$ que leva a curva $\Omega$ no círculo unitário preservando a orientação.

Mais ainda, o homeomorfismo $\Phi$ leva a região não limitada (resp. limitada), ou seja, a região exterior (resp. interior) da curva $\Omega$, na região não 
limitada (resp. limitada) do círculo unitário. É claro que o Teorema 5.3.1 continua sendo válido se trocamos o plano $\mathbb{R}^{2}$ pelo anel $\mathbb{A}$. Para uma prova elementar deste teorema pode-se ver [5].

Desse modo, a idéia da extensão segue da seguinte forma:

Observe que os $\operatorname{arcos} \gamma, \ldots, h^{q+q^{\prime}-1}(\gamma)$ dividem ao anel $\mathbb{A}$ em $q+q^{\prime}$ discos topológicos fechados, nos quais o $\operatorname{arco} h^{k}(\gamma)$ pertence à fronteira de exatamente dois deles. Similarmente acontece com os arcos $\gamma_{0}, \ldots, R_{\alpha}^{q+q^{\prime}-1}\left(\gamma_{0}\right)$, como foi demonstrado no Lema 5.2. Então, usando repetidamente o Teorema de Jordan - Schoenflies entre cada um dos discos formados pelos iterados do arco $\gamma$ por $h$ e os iterados correspondentes do arco $\gamma_{0}$ por $R_{\alpha}$, vamos conseguir um homeomorfismo do anel $\mathbb{A}$ isotópico à identidade que leve o arco $h^{k}(\gamma)$ ao arco $R_{\alpha}^{k}\left(\gamma_{0}\right)$

Assim, denominemos por $\widetilde{h}$ qualquer levantamento do homeomorfismo $h$. Analogamente, denominemos por $\Gamma$ o arco essencial simples em $\widetilde{\mathbb{A}}$ tal que $\gamma=$ $\tilde{\pi}(\Gamma)$. Comecemos a extensão nos discos formados pela tripla $\left(h^{q^{\prime}}(\gamma), \gamma, h^{q}(\gamma)\right)$. Denominemos

$$
\begin{aligned}
\widetilde{E} & :=R(\Gamma) \backslash \operatorname{Int}\left(R\left(T^{-p} \circ \widetilde{h}^{q}(\Gamma)\right)\right), \\
\widetilde{E}^{\prime} & :=R\left(T^{-p^{\prime}} \circ \widetilde{h}^{q^{\prime}}(\Gamma)\right) \backslash \operatorname{Int}(R(\Gamma)) .
\end{aligned}
$$

Portanto, os discos formados pela tripla anterior serão $E:=\widetilde{\pi}(\widetilde{E})$ e $E^{\prime}:=$ $\widetilde{\pi}\left(\widetilde{E}^{\prime}\right)$. Logo, a tripla correspondente será $\left(R_{\alpha}^{q^{\prime}}\left(\gamma_{0}\right), \gamma_{0}, R_{\alpha}^{q}\left(\gamma_{0}\right)\right)$, e portanto os discos correspondentes serão $D$ e $D^{\prime}$, os quais já foram definidos na primeira parte desta seção. Aplicando o Teorema 5.3.1, temos que existe um homeomorfismo $\Phi$ do anel $\mathbb{A}$ que leva o interior disco $E$ no interior do disco $D$, além de preservar a orientação. Similarmente, existe um homeomorfismo $\Phi^{\prime}$ do anel $\mathbb{A}$ que leva o interior do disco $E^{\prime}$ no interior do disco $D^{\prime}$ preservando a orientação. Então, o homeomorfismo $\sigma_{a}$ estende-se para o interior dos discos topológicos fechados $E$ e $E^{\prime}$ por meio dos homeomorfismos $\Phi$ e $\Phi^{\prime}$, respectivamente. 
O mesmo argumento utilizado nos discos $E$ e $E^{\prime}$ repete-se nos seguintes discos topológicos fechados do anel $\mathbb{A}$. Agora, um fato importante que justifica que a extensão do homeomorfismo $\sigma_{a}$ está bem definida é que de acordo com a Proposição 4.1, a ordem cíclica dos iterados de $\gamma$ por $h$ é a mesma para os iterados de $\gamma_{0}$ por $R_{\alpha}$. Além disso, cada um dos homeomorfismos utilizados na extensão preserva a orientação. Podemos dizer então que o homeomorfismo $\sigma_{a}$, agora estendido a todo o anel $\mathbb{A}$, é isotópico à identidade.

Definimos portanto o homeomorfismo

$$
h_{a}:=\sigma_{a} \circ h \circ \sigma_{a}^{-1}
$$

Claramente $h_{a}$ é conjugado a $h$ e isotópico à identidade. Verifiquemos que de fato $h_{a}$ coincide com a rotação $R_{\alpha}$ em cada arco $R_{\alpha}^{k}\left(\gamma_{0}\right) \operatorname{com} k \in$ $\left\{0, \ldots, q+q^{\prime}-2\right\}$.

Comecemos fixando um número inteiro $k \in\left\{0, \ldots, q+q^{\prime}-2\right\}$. Seja agora $x$ qualquer ponto que pertence ao arco $R_{\alpha}^{k}\left(\gamma_{0}\right)$. O objetivo é mostrar que

$$
\left(\sigma_{a} \circ h \circ \sigma_{a}^{-1}\right)(x)=R_{\alpha}(x)
$$

Primeiramente, pela definição de $\sigma_{a}$ temos

$$
\begin{aligned}
\sigma_{a}^{-1}: \bigcup_{k=0}^{q+q^{\prime}-1} R_{\alpha}^{k}\left(\gamma_{0}\right) & \longrightarrow \bigcup_{k=0}^{q+q^{\prime}-1} h^{k}(\gamma), \\
x \in R_{\alpha}^{k}\left(\gamma_{0}\right) & \longmapsto\left(\left.h^{k} \circ \sigma_{a}^{-1}\right|_{\gamma} \circ R_{\alpha}^{-k}\right)(x) .
\end{aligned}
$$

Portanto, $\sigma_{a}^{-1}(x) \in h^{k}(\gamma)$, daí que $\left(h \circ \sigma_{a}^{-1}\right)(x)$ pertence ao arco $h^{k+1}(\gamma)$, onde $(k+1) \in\left\{0, \ldots, q+q^{\prime}-1\right\}$.

Mais uma vez, pela definição de $\sigma_{a}$ e $\sigma_{a}^{-1}$, obtém-se finalmente 


$$
\begin{aligned}
\left(\sigma_{a} \circ h \circ \sigma_{a}^{-1}\right)(x) & =\left(\left(\left.R_{\alpha}^{k+1} \circ \sigma_{a}\right|_{\gamma} \circ h^{-(k+1)}\right) \circ\left(h \circ \sigma_{a}^{-1}\right)\right)(x), \\
& =\left(\left(\left.R_{\alpha}^{k+1} \circ \sigma_{a}\right|_{\gamma} \circ h^{-(k+1)}\right) \circ h \circ\left(\left.h^{k} \circ \sigma_{a}^{-1}\right|_{\gamma} \circ R_{\alpha}^{-k}\right)\right)(x), \\
\left(\sigma_{a} \circ h \circ \sigma_{a}^{-1}\right)(x) & =R_{\alpha}(x) .
\end{aligned}
$$

Isto termina a primeira parte da prova da Proposição 5.1.

Antes de começar a segunda parte da prova, note que

$$
d\left(\sigma_{a} \circ h \circ \sigma_{a}^{-1}, R_{\alpha}\right) \leq \sqrt{2} .
$$

Assim, a Proposição 5.1 ficaria demonstrada nesta primeira parte se o $\min \left(q, q^{\prime}\right) \leq 28$. Denotando por

$$
s=\min \left(q, q^{\prime}\right)
$$

suponhamos a partir de agora que $s>28$.

\subsubsection{Segunda parte da prova}

Nesta segunda parte construiremos um homeomorfismo $\sigma_{b}$ definido no anel $\mathbb{A}$, isotópico à identidade, tal que o conjugado $h_{b}:=\sigma_{b} \circ h_{a} \circ \sigma_{b}^{-1}$ coincide com a rotação $R_{\alpha}$ em qualquer ponto, exceto possivelmente no disco topológico $O^{\prime}:=R_{\alpha}^{-1}(O)=R_{\alpha}^{q^{\prime}-1}(D) \cup R_{\alpha}^{q-1}\left(D^{\prime}\right)$.

Comecemos descrevendo uma conseqüência da primeira parte.

Observação 5.7 O homeomorfismo $h_{a}^{k}$ coincide com a rotação $R_{\alpha}^{k}$ no arco $\gamma_{0}$ com $k \in\left\{0, \ldots, q+q^{\prime}-1\right\}$.

Demonstração. Em particular, no caso em que $k=0$ a observação é obvia, e se $k=1$ ela está justificada pela primeira parte. Por outro lado, suponhamos que nossa observação é verdadeira para $k \in\left\{1, \ldots, q+q^{\prime}-1\right\}$. Seja $x$ qualquer ponto que pertence ao arco $\gamma_{0}$. Temos 


$$
h_{a}^{k+1}(x)=h_{a}\left(h_{a}^{k}(x)\right)=h_{a}\left(R_{\alpha}^{k}(x)\right) .
$$

Como $R_{\alpha}^{k}(x) \in R_{\alpha}^{k}\left(\gamma_{0}\right)$, temos pela primeira parte que $h_{a}\left(R_{\alpha}^{k}(x)\right)=$ $R_{\alpha}\left(R_{\alpha}^{k}(x)\right)$. Desse modo,

$$
h_{a}^{k+1}(x)=R_{\alpha}^{k+1}(x) .
$$

Esta observação permite dizer que

$$
h_{a}^{k}\left(\gamma_{0}\right)=R_{\alpha}^{k}\left(\gamma_{0}\right)
$$

para todo $k \in\left\{0, \ldots, q+q^{\prime}-1\right\}$. Ainda, junto com o fato de $h_{a}$ e $R_{\alpha}$ serem homeomorfismos isotópicos à identidade, podemos estender (5.15) aos discos $D$ e $D^{\prime}$, tal como descreve a seguinte observação:

Observação 5.8 Sejam $D$ e $D^{\prime}$ os discos topológicos fechados definidos na primeira parte da Seção 5.3. Então temos

$$
\begin{aligned}
h_{a}^{k}(D) & =R_{\alpha}^{k}(D), & k=\left\{0, \ldots, q^{\prime}-1\right\}, \\
h_{a}^{k^{\prime}}\left(D^{\prime}\right) & =R_{\alpha}^{k^{\prime}}\left(D^{\prime}\right), & k^{\prime}=\{0, \ldots, q-1\} .
\end{aligned}
$$

Demonstração. Vamos demonstrar só a primeira expressão, pois a segunda segue por analogia.

Denotemos por $C_{1}$ a fronteira do disco $D$. Note que, como variedade topológica, $C_{1}$ vai ser uma curva fechada simples, tal que

$$
C_{1}=\gamma_{0} \cup R_{\alpha}^{q}\left(\gamma_{0}\right) \cup C_{1}^{+} \cup C_{1}^{-},
$$

onde $C_{1}^{+}=\tilde{\pi}([0, q \alpha-p] \times\{1\})$ e $C_{1}^{-}=\widetilde{\pi}([0, q \alpha-p] \times\{0\})$.

Fixando um inteiro $k \in\left\{0, \ldots, q^{\prime}-1\right\}$, a imagem da fronteira $C_{1}$ pelos homeomorfismos $h_{a}^{k}$ e a rotação $R_{\alpha}^{k}$ é 


$$
\begin{aligned}
& h_{a}^{k}\left(C_{1}\right)=h_{a}^{k}\left(\gamma_{0}\right) \cup h_{a}^{k}\left(R_{\alpha}^{q}\left(\gamma_{0}\right)\right) \cup h_{a}^{k}\left(C_{1}^{+}\right) \cup h_{a}^{k}\left(C_{1}^{-}\right), \\
& R_{a}^{k}\left(C_{1}\right)=R_{\alpha}^{k}\left(\gamma_{0}\right) \cup R_{\alpha}^{k}\left(R_{\alpha}^{q}\left(\gamma_{0}\right)\right) \cup R_{\alpha}^{k}\left(C_{1}^{+}\right) \cup R_{\alpha}^{k}\left(C_{1}^{-}\right) .
\end{aligned}
$$

Suponhamos por instante que $h_{a}^{k}\left(C_{1}\right)=R_{\alpha}^{k}\left(C_{1}\right)$. Como $h_{a}^{k}$ e $R_{\alpha}^{k}$ são homeomorfismos que preservam a orientação do anel $\mathbb{A}$, então

$$
\begin{aligned}
h_{a}^{k}(\operatorname{int} D) & =\operatorname{int}\left(h_{a}^{k}(D)\right) \\
R_{\alpha}^{k}(\operatorname{int} D) & =\operatorname{int}\left(R_{\alpha}^{k}(D)\right)
\end{aligned}
$$

Mas, $h_{a}^{k}(D)$ (resp. $R_{\alpha}^{k}(D)$ ) possui como fronteira a curva $h_{a}^{k}\left(C_{1}\right)$ (resp. $\left.R_{\alpha}^{k}\left(C_{1}\right)\right)$. Logo segue da nossa suposição que $h_{a}^{k}(D)=R_{\alpha}^{k}(D)$.

Mostremos então que $h_{a}^{k}\left(C_{1}\right)=R_{\alpha}^{k}\left(C_{1}\right)$. Por (5.15) é fácil ver que

$$
h_{a}^{k}\left(\gamma_{0}\right)=R_{\alpha}^{k}\left(\gamma_{0}\right)
$$

Similarmente,

$$
h_{a}^{k}\left(R_{\alpha}^{q}\left(\gamma_{0}\right)\right)=R_{\alpha}^{k}\left(R_{\alpha}^{q}\left(\gamma_{0}\right)\right)
$$

Falto só verificar que $h_{a}^{k}\left(C_{1}^{+}\right)=R_{\alpha}^{k}\left(C_{1}^{+}\right)$e $h_{a}^{k}\left(C_{1}^{-}\right)=R_{\alpha}^{k}\left(C_{1}^{-}\right)$. Para isto, note que os pontos extremos da curva fechada $C_{1}^{+}$são os pontos $(0,1)$ e $(q \alpha-p, 1)=R_{\alpha}^{q}((0,1))$, onde $(0,1) \in \gamma_{0}$. Pela Observação 5.7, temos

$$
h_{a}^{k}(0,1)=R_{\alpha}^{k}(0,1)
$$

De igual forma,

$$
h_{a}^{k}(q \alpha-p, 1)=R_{\alpha}^{k}(q \alpha-p, 1) .
$$

Portanto, $h_{a}^{k}$ e $R_{\alpha}^{k}$ coincidem nos pontos extremos da curva fechada $C_{1}^{+}$. Por outro lado, os homeomorfismos $h_{a}$ e $R_{\alpha}^{k}$ preservam as componentes da fronteira (ver item (ii) da Proposição 2.1), ou seja, 


$$
\begin{aligned}
& h_{a}^{k}\left(C_{1}^{+}\right) \in \mathbb{S}^{1} \times\{1\}, \\
& R_{\alpha}^{k}\left(C_{1}^{+}\right) \in \mathbb{S}^{1} \times\{1\} .
\end{aligned}
$$

Daí, junto com o fato de preservarem a orientação do anel $\mathbb{A}$, concluímos que $h_{a}^{k}\left(C_{1}^{+}\right)=R_{\alpha}^{k}\left(C_{1}^{+}\right)$. Do mesmo jeito pode-se mostrar que $h_{a}^{k}\left(C_{1}^{-}\right)=$ $R_{\alpha}^{k}\left(C_{1}^{-}\right)$.

Vamos construir então o homeomorfismo $\sigma_{b}$.

Para cada $k \in\left\{0, \ldots, q^{\prime}-1\right\}$, definimos a aplicação $\sigma_{b}$ no disco $R_{\alpha}^{k}(D)$ como

$$
\sigma_{b}:=R_{\alpha}^{k} \circ h_{a}^{-k}
$$

Similarmente, para cada $k^{\prime} \in\{0, \ldots, q-1\}$, a mesma fórmula será usada no disco $R_{\alpha}^{k^{\prime}}\left(D^{\prime}\right)$. Agora, pela Observação 5.8, temos $D=h_{a}^{-k} \circ R_{\alpha}^{k}(D)$. Isto implica que $\sigma_{b}\left(R_{\alpha}^{k}(D)\right)=R_{\alpha}^{k}(D)$. Analogamente, $\sigma_{b}\left(R_{\alpha}^{k^{\prime}}\left(D^{\prime}\right)\right)=R_{\alpha}^{k^{\prime}}\left(D^{\prime}\right)$. Por outro lado, no Lema 5.2, vimos que a interseção dos discos $R_{\alpha}^{k}(D)$ e $R_{\alpha}^{k^{\prime}}\left(D^{\prime}\right)$ ou era vazia ou era um $\operatorname{dos} \operatorname{arcos} R_{\alpha}^{\ell}\left(\gamma_{0}\right) \operatorname{com} \ell \in\left\{0, \ldots, q+q^{\prime}-1\right\}$. Mas, pela Observação 5.7, $R_{\alpha}^{k} \circ h_{a}^{-k}$ é igual à identidade nesses arcos. Portanto, $\sigma_{b}$ está bem definido. Adicionalmente, pelo fato de que $R_{\alpha}^{k}$ e $h_{a}^{k}$ são isotópicos à identidade, a aplicação $\sigma_{b}$ será também um homeomorfismo do anel $\mathbb{A}$ isotópico à identidade.

Dessa maneira, é fácil mostrar que o conjugado $h_{b}$, definido no início desta subseção, coincide com a rotação $R_{\alpha}$ em cada disco $R_{\alpha}^{k}(D) \operatorname{com} k \in$ $\left\{0, \ldots, q^{\prime}-2\right\}$ e $R_{\alpha}^{k^{\prime}}\left(D^{\prime}\right)$ com $k^{\prime} \in\{0, \ldots, q-2\}$. Por exemplo, fixemos um inteiro $k \in\left\{0, \ldots, q^{\prime}-2\right\}$. Seja $x$ qualquer ponto que pertence ao disco $R_{\alpha}^{k}(D)$. Pela definição de $h_{b}$ temos

$$
h_{b}(x)=\left(\sigma_{b} \circ h_{a} \circ \sigma_{b}^{-1}\right)(x) .
$$

Agora, se $x \in R_{\alpha}^{k}(D)$, então 


$$
\sigma_{b}^{-1}(x)=\left(h_{a}^{k} \circ R_{\alpha}^{-k}\right)(x)
$$

tal que $\sigma_{b}^{-1}(x) \in R_{\alpha}^{k}(D)$.

A Observação 5.8 implica que

$$
\begin{aligned}
h_{a}\left(R_{\alpha}^{k}(D)\right) & =R_{\alpha}\left(R_{\alpha}^{k}(D)\right), & & k=\left\{0, \ldots, q^{\prime}-2\right\}, \\
h_{a}\left(R_{\alpha}^{k^{\prime}}\left(D^{\prime}\right)\right) & =R_{\alpha}\left(R_{\alpha}^{k^{\prime}}\left(D^{\prime}\right)\right), & & k^{\prime}=\{0, \ldots, q-2\} .
\end{aligned}
$$

Daí, $\left(h_{a} \circ \sigma_{b}^{-1}\right)(x)$ pertence ao disco $R_{\alpha}^{k+1}(D)$. Logo, usando a definição do homeomorfismo $\sigma_{b}$ obtemos finalmente

$$
\begin{aligned}
& \left(\sigma_{b} \circ h_{a} \circ \sigma_{b}^{-1}\right)(x)=\left(\left(R_{\alpha}^{k+1} \circ h_{a}^{-(k+1)}\right) \circ h_{a} \circ\left(h_{a}^{k} \circ R_{\alpha}^{-k}\right)\right), \\
& \left(\sigma_{b} \circ h_{a} \circ \sigma_{b}^{-1}\right)(x)=R_{\alpha}(x) .
\end{aligned}
$$

De modo análogo mostra-se o caso no disco $R_{\alpha}^{k^{\prime}}\left(D^{\prime}\right)$ com $k^{\prime} \in\{0, \ldots, q-2\}$.

Utilizando o Lema 5.2, vemos que a união de todos estes discos cobrem o anel todo menos o conjunto $O^{\prime}=R_{\alpha}^{q^{\prime}-1}(D) \cup R_{\alpha}^{q-1}\left(D^{\prime}\right)$.

Isto termina a segunda parte da prova da Proposição 5.1.

\subsubsection{Terceira parte da prova}

Nesta última parte da prova definiremos o homeomorfismo $g:=R_{\alpha}^{-1} \circ h_{b}$ no conjunto $O^{\prime}$, para assim poder aplicar nele o resultado da decomposição dos homeomorfismos do disco. Com isso, construiremos um homeomorfismo $\sigma_{c}$ definido no anel $\mathbb{A}$, isotópico à identidade, tal que o conjugado $h_{c}:=$ $\sigma_{c} \circ h_{b} \circ \sigma_{c}^{-1}$ seja $\epsilon$-próximo da identidade, com $\epsilon \leq 40 / s$ (lembre-se que $\left.s=\min \left(q, q^{\prime}\right)\right)$.

Consideremos então o homeomorfismo $g$ do disco topológico $O^{\prime}$ definido como $g=R_{\alpha}^{-1} \circ h_{b}$. Note que os arcos que pertencem à fronteira do disco $O^{\prime}$ são $R_{\alpha}^{q-1}\left(\gamma_{0}\right)$ e $R_{\alpha}^{q^{\prime}-1}\left(\gamma_{0}\right)$. É fácil verificar que $h_{b}$ coincide com a rotação $R_{\alpha}$ nestes 
arcos; temos assim que $g$ é igual à identidade nos $\operatorname{arcos} R_{\alpha}^{q-1}\left(\gamma_{0}\right)$ e $R_{\alpha}^{q^{\prime}-1}\left(\gamma_{0}\right)$. Além disso, pelo fato de $R_{\alpha}^{-1}$ e $h_{b}$ serem isotópicos à identidade, é claro que o homeomorfismo $g$ vai ser isotópico à identidade também. Portanto, $g \in$ Homeo $^{+}\left(O^{\prime}, R_{\alpha}^{q-1}\left(\gamma_{0}\right) \cup R_{\alpha}^{q^{\prime}-1}\left(\gamma_{0}\right)\right)$.

Seja $\epsilon>0$, onde

$$
\epsilon=\frac{2(4+2 \pi)}{s-7} .
$$

De acordo com a Observação 5.6, $g$ pode ser escrito como

$$
g=g_{N} \circ \cdots \circ g_{1}
$$

tal que

$$
N<2\left(\frac{4+2 \pi}{\epsilon}\right)+5=s-2,
$$

e onde cada $g_{i}$ é um homeomorfismos do disco $O^{\prime}$ que é igual à identidade em $R_{\alpha}^{q-1}\left(\gamma_{0}\right) \cup R_{\alpha}^{q^{\prime}-1}\left(\gamma_{0}\right)$, e ainda é $\epsilon$-próximo da identidade.

Uma vez que assumimos $s=\min \left(q, q^{\prime}\right)$ maior do que 28 , vemos que

$$
\begin{aligned}
\epsilon-\frac{40}{s} & =\frac{2(4+2 \pi)}{s-8}-\frac{40}{s} \\
& =\frac{320-(32-4 \pi) s}{s(s-8)} \\
& \leq 0
\end{aligned}
$$

Daí,

$$
\epsilon \leq \frac{40}{s} .
$$

Agora, para cada $k \in\{1, \ldots, N\}$, definimos $\sigma_{c}$ no disco $R_{\alpha}^{k}\left(O^{\prime}\right)$ pela fórmula

$$
\sigma_{c}:=R_{\alpha}^{k} \circ g_{k} \circ \cdots \circ g_{1} \circ h_{b}^{-k} .
$$


No resto do anel $\mathbb{A}, \sigma_{c}$ será igual à identidade.

Vamos então verificar que $\sigma_{c}$ está bem definido, e que o homeomorfismo $h_{c}$ definido como $\sigma_{c} \circ h_{b} \circ \sigma_{c}^{-1}$ é $\epsilon$-próximo da identidade. Para isto, comecemos descrevendo uma conseqüência da segunda parte da prova.

Observação 5.9 Para cada $k \in\{1, \ldots, s\}$,

$$
h_{b}^{k}\left(O^{\prime}\right)=R_{\alpha}^{k}\left(O^{\prime}\right)
$$

Demonstração. Pela definição do homeomorfismo $g$ temos que $\left(R_{\alpha}^{-1} \circ\right.$ $\left.h_{b}\right)\left(O^{\prime}\right)=O^{\prime}$. Portanto,

$$
h_{b}\left(O^{\prime}\right)=R_{\alpha}\left(O^{\prime}\right)
$$

Agora, suponhamos que a afirmação seja verdadeira para $k \in\{1, \ldots, s-1\}$. Assim,

$$
h_{b}^{k+1}\left(O^{\prime}\right)=h_{b}\left(h_{b}^{k}\left(O^{\prime}\right)\right)=h_{b}\left(R_{\alpha}^{k}\left(O^{\prime}\right)\right)
$$

Como $O^{\prime}=R_{\alpha}^{-1}(D) \cup R_{\alpha}^{-1}\left(D^{\prime}\right)$, então $R_{\alpha}^{k}\left(O^{\prime}\right)=R_{\alpha}^{k-1}(D) \cup R_{\alpha}^{k-1}\left(D^{\prime}\right)$, tal que $(k-1) \in\{0, \ldots, s-2\}$. O homeomorfismo $h_{b}$ coincide com $R_{\alpha}$ em cada disco $R_{\alpha}^{i}(D)$ com $i \in\left\{0, \ldots, q^{\prime}-2\right\}$ e $R_{\alpha}^{j}(D)$ com $j \in\{0, \ldots, q-2\}$, e $(k-1) \in\left\{0, \ldots, q^{\prime}-2\right\} \cap\{0, \ldots, q-2\}$ ( pois $\left.s=\min \left(q, q^{\prime}\right)\right)$. Disso,

$$
h_{b}\left(R_{\alpha}^{k}\left(O^{\prime}\right)\right)=R_{\alpha}\left(R_{\alpha}^{k}\left(O^{\prime}\right)\right)=R_{\alpha}^{k+1}\left(O^{\prime}\right) .
$$

Sendo $N<s-2$, para cada $k \in\{1, \ldots, N\}$, a observação anterior garante que no disco $R_{\alpha}^{k}\left(O^{\prime}\right)$, vale: $h_{b}^{-k}\left(R_{\alpha}^{k}\left(O^{\prime}\right)\right)=O^{\prime}$. Por outro lado, da definição dos homeomorfismos $g_{i}^{\prime} s$ podemos dizer que $\left(g_{k} \circ \cdots \circ g_{1}\right)\left(O^{\prime}\right)=O^{\prime}$. Estes dois fatos implicam que 


$$
\begin{aligned}
\sigma_{c}\left(R_{\alpha}^{k}\left(O^{\prime}\right)\right) & =\left(R_{\alpha}^{k} \circ g_{k} \circ \cdots \circ g_{1} \circ h_{b}^{-k}\right)\left(R_{\alpha}^{k}\left(O^{\prime}\right)\right) \\
& =\left(R_{\alpha}^{k} \circ g_{k} \circ \cdots \circ g_{1}\right)\left(O^{\prime}\right) \\
& =R_{\alpha}^{k}\left(O^{\prime}\right)
\end{aligned}
$$

Resta só verificar se $\sigma_{c}$ coincide com a identidade nos arcos que pertencem à fronteira do disco $R_{\alpha}^{k}\left(O^{\prime}\right)$. Para garantir isso, descrevemos mais uma conseqüência da segunda parte da prova.

Observação 5.10 Para cada $k \in\{1, \ldots, s\}$, o homeomorfismo $h_{b}^{k}$ coincide com $R_{\alpha}^{k}$ nos arcos que pertencem à fronteira do disco $O^{\prime}$. Neste caso, $R_{\alpha}^{q-1}\left(\gamma_{0}\right)$ e $R_{\alpha}^{q^{\prime}-1}\left(\gamma_{0}\right)$.

Demonstração. Seja $x$ qualquer ponto do arco $R_{\alpha}^{q-1}\left(\gamma_{0}\right)$. Pela definição do homeomorfismo $g$, temos que $h_{b}(x)=R_{\alpha}(x)$. Suponhamos agora que a afirmação seja verdadeira para $k \in\{1, \ldots, s-1\}$. Assim,

$$
h_{b}^{k+1}(x)=h_{b}\left(h_{b}^{k}(x)\right)=h_{b}\left(R_{\alpha}^{k}(x)\right) .
$$

Pela segunda parte da prova, $h_{b}$ coincide com $R_{\alpha}$ no arco $R_{\alpha}^{i+q}\left(\gamma_{0}\right)$ com $i \in\left\{0, \ldots, q^{\prime}-2\right\}\left(R_{\alpha}^{q}\left(\gamma_{0}\right)\right.$ pertence à fronteira do disco $\left.D\right)$. Por outro lado, $R_{\alpha}^{k}(x) \in R_{\alpha}^{(k-1)+q}\left(\gamma_{0}\right)$, tal que $(k-1) \in\{0, \ldots, s-2\} \subset\left\{0, \ldots, q^{\prime}-2\right\}$. Portanto,

$$
\left.h_{b}\left(R_{\alpha}^{k}(x)\right)=R_{\alpha}\left(R_{\alpha}^{k}(x)\right)=R_{\alpha}^{k+1}(x)\right) .
$$

No caso em que $x \in R_{\alpha}^{q^{\prime}-1}\left(\gamma_{0}\right)$ a demonstração é similar.

Desse modo, fixando um inteiro $k \in\{1, \ldots, N\}$, os arcos que pertencem à fronteira do disco $R_{\alpha}^{k}\left(O^{\prime}\right)$ são $R_{\alpha}^{k+(q-1)}\left(\gamma_{0}\right)$ e $R_{\alpha}^{k+\left(q^{\prime}-1\right)}\left(\gamma_{0}\right)$. Seja então $x$ qualquer ponto do arco $R_{\alpha}^{k+(q-1)}\left(\gamma_{0}\right)$. É claro que $x=R_{\alpha}^{k}(y)$, onde $y \in$ $R_{\alpha}^{q-1}\left(\gamma_{0}\right)$. Como $N<s-2$, a observação anterior garante $x=h_{b}^{k}(y)$. Daí

$$
h_{b}^{-k}(x)=h_{b}^{-k}\left(h_{b}^{k}(y)\right)=y .
$$


Ou seja, $h_{b}^{-k}(x)$ pertence ao arco $R_{\alpha}^{q-1}\left(\gamma_{0}\right)$. Por outro lado, o homeomorfismo $g_{i}$ coincide com a identidade em $R_{\alpha}^{q-1}\left(\gamma_{0}\right) \cup R_{\alpha}^{q^{\prime}-1}\left(\gamma_{0}\right)$. Destes dois fatos decorre facilmente que

$$
\begin{aligned}
\sigma_{c}(x) & =\left(R_{\alpha}^{k} \circ g_{k} \circ \cdots \circ g_{1} \circ h_{b}^{-k}\right)(x), \\
& =\left(R_{\alpha}^{k} \circ g_{k} \circ \cdots \circ g_{1}\right)(y), \\
& =R_{\alpha}^{k}(y), \\
& =x .
\end{aligned}
$$

Analagomamente, o raciocínio é o mesmo se $x \in R_{\alpha}^{k+\left(q^{\prime}-1\right)}\left(\gamma_{0}\right)$. Podemos verificar então que $\sigma_{c}$ é igual à identidade nos $\operatorname{arcos} R_{\alpha}^{\ell}\left(\gamma_{0}\right)$ que pertencem à fronteira destes discos. Isto prova que $\sigma_{c}$ está bem definido no anel $\mathbb{A}$. Ainda, pelas características dos homeomorfismos $h_{b}^{k}, R_{\alpha}^{k}$ e $g_{i}$, é claro que $\sigma_{c}$ vai ser isotópico à identidade.

Antes de verificar que o homeomorfismo $h_{c}$ é $\epsilon$-próximo da identidade, note primeiro que $\sigma_{c}$ é igual a $R_{\alpha}^{N} \circ g \circ h_{b}^{-N}$ no disco $R_{\alpha}^{N}\left(O^{\prime}\right)$. Por outro lado, pela segunda parte da prova é fácil mostrar que para cada $k \in\{0, \ldots, s-1\}$ o homeomorfismo $h_{b}^{k}$ coincide com $R_{\alpha}^{k}$ no disco $R_{\alpha}\left(O^{\prime}\right)=D \cup D^{\prime}$ (utilizando seguir a mesma idéia da Observação 5.7). Então, dado que $N<s-1$, temos

$$
h_{b}^{N}=R_{b}^{N} \circ g,
$$

no disco $O^{\prime}$. De fato, seja $x$ qualquer ponto do disco $O^{\prime}$. Como $g(x) \in O^{\prime}$ então $\left(R_{\alpha} \circ g\right)(x) \in R_{\alpha}\left(O^{\prime}\right)$. Utilizando a afirmação anterior junto com a definição de $g$ temos

$$
\begin{aligned}
\left(R_{\alpha}^{N} \circ g\right)(x) & =R_{\alpha}^{N-1}\left(\left(R_{\alpha} \circ g\right)(x)\right) \\
& =h_{b}^{N-1}\left(\left(R_{\alpha} \circ R_{\alpha}^{-1} \circ h_{b}\right)(x)\right), \\
& =h_{b}^{N}(x) .
\end{aligned}
$$

Podemos dizer então que $\sigma_{c}$ é igual à identidade no disco $R_{\alpha}^{N}\left(O^{\prime}\right)$. 
Desse modo, para verificar que o homeomorfismo $h_{c}$ é $\epsilon$-próximo da identidade, vamos mostrar primeiramente o seguinte resultado:

Observação 5.11 Para cada $k \in\{0, \ldots, N-1\}$, o homeomorfismo $h_{c}$ é igual $a R_{\alpha} \circ\left(R_{\alpha}^{k} \circ g_{k+1} \circ R_{\alpha}^{-k}\right)$ no disco $R_{\alpha}^{k}\left(O^{\prime}\right)$.

Demonstração. Para $k=0$, vemos que para qualquer ponto $x$ do disco $O^{\prime}$ temos que $\sigma_{c}^{-1}(x)=x \in O^{\prime}$ pois $\sigma_{c}$ coincide com a identidade neste disco. Como $h_{b}\left(O^{\prime}\right)=R_{\alpha}\left(O^{\prime}\right)$, vemos que $h_{b}\left(\sigma_{c}^{-1}(x)\right) \in R_{\alpha}\left(O^{\prime}\right)$.

Aplicando a definição de $\sigma_{c}$ obtemos

$$
\begin{aligned}
h_{c}(x) & =\left(\sigma_{c} \circ h_{b} \circ \sigma_{c}^{-1}\right)(x), \\
& =\left(R_{\alpha} \circ g_{1} \circ h_{b}^{-1}\right)\left(h_{b}\left(\sigma_{c}^{-1}(x)\right)\right), \\
& =\left(R_{\alpha} \circ g_{1} \circ h_{b}^{-1}\right)\left(h_{b}(x)\right), \\
& =\left(R_{\alpha} \circ g_{1}\right)(x) .
\end{aligned}
$$

Suponhamos então que $k \in\{1, \ldots, N-1\}$. Seja $x$ qualquer ponto do disco $R_{\alpha}^{k}\left(O^{\prime}\right)$. Então

$$
\sigma_{c}^{-1}(x)=\left(h_{b}^{k} \circ g_{1}^{-1} \circ \cdots g_{k}^{-1} \circ R_{\alpha}^{-k}\right)(x),
$$

o que implica que $\sigma_{c}^{-1}(x) \in R_{\alpha}^{k}\left(O^{\prime}\right)$. Agora, dado que $N<s-2$, pela segunda parte da prova segue que $h_{b}\left(\sigma_{c}^{-1}(x)\right)=R_{\alpha}\left(\sigma_{c}^{-1}(x) \in R_{\alpha}^{k+1}\left(O^{\prime}\right)\right.$, onde $(k+1) \in\{2, \ldots, N\}$. Aplicando a definição de $\sigma_{c}$ obtemos finalmente

$$
\begin{aligned}
h_{c}(x) & =\left(\sigma_{c} \circ h_{b} \circ \sigma_{c}^{-1}\right)(x), \\
& =\left(R_{\alpha}^{k+1} \circ g_{k+1} \circ \cdots \circ g_{1} \circ h_{b}^{-(k+1)}\right)\left(h_{b}\left(\sigma_{c}^{-1}(x)\right)\right), \\
& =\left(\left(R_{\alpha}^{k+1} \circ g_{k+1} \circ \cdots \circ g_{1} \circ h_{b}^{-(k+1)}\right) \circ h_{b} \circ\left(h_{b}^{k} \circ g_{1}^{-1} \circ \cdots g_{k}^{-1} \circ R_{\alpha}^{-k}\right)\right)(x), \\
& =\left(R_{\alpha}^{k+1} \circ g_{k+1} \circ R_{\alpha}^{-k}\right)(x), \\
& =\left(R_{\alpha} \circ\left(R_{\alpha}^{k} \circ g_{k+1} \circ R_{\alpha}^{-k}\right)\right)(x) .
\end{aligned}
$$


Dessa forma, seja $y$ qualquer ponto do disco $R_{\alpha}^{k}\left(O^{\prime}\right) \operatorname{com} k \in\{0, \ldots, N-1\}$. Então existe um único ponto $x \in O^{\prime}$ tal que $x=R_{\alpha}^{-k}(y)$. Assim,

$$
d\left(g_{k+1}(x), x\right)=d\left(g_{k+1}\left(R_{\alpha}^{-k}(y)\right), R_{\alpha}^{-k}(y)\right) .
$$

Mas $g_{k+1}$ é $\epsilon$-próximo da identidade no disco $O^{\prime}$, portanto

$$
d\left(g_{k+1}\left(R_{\alpha}^{-k}(y)\right), R_{\alpha}^{-k}(y)\right)<\epsilon .
$$

Dado que $R_{\alpha}$ é uma isometria, segue que

$$
d\left(\left(R_{\alpha}^{k+1} \circ g_{k+1} \circ R_{\alpha}^{-k}\right)(y), R_{\alpha}(y)\right)=d\left(g_{k+1}\left(R_{\alpha}^{-k}(y)\right), R_{\alpha}^{-k}(y)\right)<\epsilon .
$$

Pela Observação anterior, resulta então

$$
\left.d\left(h_{c}(y), R_{\alpha}(y)\right)=d\left(\left(R_{\alpha}^{k+1} \circ g_{k+1} \circ R_{\alpha}^{-k}\right)(y)\right), R_{\alpha}(y)\right)<\epsilon,
$$

onde $y \in R_{\alpha}^{k}\left(O^{\prime}\right)$.

Podemos dizer assim que $h_{c}$ é $\epsilon$-próximo à rotação $R_{\alpha}$ no conjunto $\bigcup_{k=0}^{N-1} R_{\alpha}^{k}\left(O^{\prime}\right)$. Além disso, por definição, o homeomorfismo $\sigma_{c}$ coincide com a identidade no conjunto $E:=\mathbb{A} \backslash \bigcup_{k=0}^{N-1} R_{\alpha}^{k}\left(O^{\prime}\right)$, (lembre-se que $\sigma_{c}=$ id também no disco $\left.R_{\alpha}^{N}\left(O^{\prime}\right)\right)$. Logo, $h_{c}$ vai coincidir com $h_{b}$ no conjunto $E$ por definição. De acordo com a segunda parte da prova, sabemos que $h_{b}$ é igual à rotação $R_{\alpha}$ no conjunto $E$, portanto $h_{c}$ vai coincidir com a rotação $R_{\alpha}$ neste conjunto. Concluímos então que a conjugação $h_{c}$ é $\epsilon$-próximo à rotação $R_{\alpha}$ no anel $\mathbb{A}$. Ainda $h_{c}$ vai ser isotópico à identidade pelo fato dos homeomorfismo $\sigma_{c}$ e $h_{b}$ serem isotópicos à identidade também.

Finalmente, denotemos por $\sigma:=\sigma_{c} \circ \sigma_{b} \circ \sigma_{a}$ o homeomorfismo do anel $\mathbb{A}$, isotópico à identidade. De nossa última afirmação e (5.16) obtemos

$$
d\left(\sigma \circ h \circ \sigma^{-1}, R_{\alpha}\right)=d\left(\sigma_{c} \circ h_{b} \circ \sigma_{c}^{-1}, R_{\alpha}\right)<\epsilon \leq \frac{40}{\min \left(q, q^{\prime}\right)} .
$$

Isto termina a prova da Proposição 5.1. 


\section{Capítulo 6}

\section{Prova do Corolário 1.2}

O Teorema 1.1, demonstrado mediante duas formas diferentes nos Capítulos 2 e 3, vai reduzir o Corolário 1.2 ao seguinte fato: o homeomorfismo que possui a propriedade de interseção de curvas (qualquer curva fechada simples homotopicamente não-trivial intersecta sua imagem) e nenhum ponto periódico é uma pseudo-rotação irracional. Para simplificar a prova deste fato utilizaremos o resultado de Bonatti-Guillou [11] junto com dois lemas que serão mostrados na seguinte seção. Em particular, no primeiro lema mudaremos a hipótese do homeomorfismo não possuir pontos periódicos pelo fato de não possuir pontos fixos, e construiremos uma curva fechada simples homotopicamente não-trivial que não intersecta sua imagem. De maneira análoga, no segundo lema vamos conseguir mostrar que para qualquer levantamento do homeomorfismo seu conjunto de rotação é disjunto do conjunto dos inteiros. 


\subsection{Resultado de Bonatti-Guillou mais dois lemas técnicos}

Nesta seção começamos descrevendo o resultado de Bonatti-Guillou cuja prova pode ser vista em [11]. Daí passaremos a descrever e mostrar o primeiro lema, no qual a construção da curva desejada segue as mesmas idéias da demonstração da Proposição 3.1. Finalmente, apresentaremos o segundo lema, em cuja prova faremos uma extensão do levantamento do homeomorfismo do anel ao todo o plano. Esta extensão terá a mesma dinâmica do levantamento. Isto permitirá utilizar o Corolário 2.2 da Teoria de Brower acerca dos homeomorfismos do plano para mostrar que nenhum inteiro está contido em seu conjunto de rotação.

Teorema 6.1 ([11]) Seja h um homeomorfismo do anel $\mathbb{A}$ o qual é isotópico à identidade. Suponhamos que h não possui nenhum ponto fixo. Então, pelo menos uma das seguintes propriedades é satisfeita:

(i) Existe uma curva fechada simples homotopicamente não trivial em $\mathbb{A}$ que é disjunta da sua imagem por $h$.

(ii) Existe um arco essencial simples em $\mathbb{A}$ que é disjunto da sua imagem por $h$.

Antes de apresentar o primeiro lema técnico, vamos descrever algumas notações e propriedades que serão utilizadas na demonstração do mesmo. Para cada curva fechada simples homotopicamente não trivial $\sigma$ em $\mathbb{A}$, denotemos por $B(\sigma)$ o fecho da componente conexa de $\mathbb{A} \backslash \sigma$ que está 'abaixo' de $\sigma$, ou seja, que contém $\mathbb{S}^{1} \times\{0\}$. Seja agora $\widetilde{\sigma}:=\widetilde{\pi}^{-1}(\sigma)$. Similarmente, denotaremos por $B(\widetilde{\sigma})$ o fecho da componente conexa de $\widetilde{\mathbb{A}} \backslash \widetilde{\sigma}$ que está 'abaixo' de $\widetilde{\sigma}$, ou seja, que contém $\mathbb{R} \times\{0\}$. 
Observação 6.1 Sejam $\sigma_{1}$ e $\sigma_{2}$ duas curvas fechadas simples homotopicamente não triviais em $\mathbb{A}$. Então a fronteira da componente conexa de $\left(\mathbb{A} \backslash B\left(\sigma_{1}\right)\right) \cap\left(\mathbb{A} \backslash B\left(\sigma_{2}\right)\right)$ que contém $\mathbb{S}^{1} \times\{1\}$ é uma curva fechada simples homotopicamente não trivial, a qual denotaremos por $\sigma_{1} \vee \sigma_{2}$.

Demonstração. A prova é similar à do Lema 3.2.

Sejam $\sigma_{1}$ e $\sigma_{2}$ duas curvas fechadas simples homotopicamente não triviais em $\mathbb{A}$, então diremos que $\sigma_{1}<\sigma_{2}$ (resp. $\left.\sigma_{1} \leq \sigma_{2}\right)$ se $\sigma_{2} \subset \operatorname{Int}\left(B\left(\sigma_{1}\right)\right.$ ) (resp. $\left.\sigma_{2} \subset B\left(\sigma_{1}\right)\right)$. Desta notação e da Observação 6.1, obtemos de maneira similar as mesmas propriedades obtidas no início da Seção 3.2 para os arcos essenciais simples, as quais são apresentadas na seguinte observação.

Observação 6.2 Sejam $\sigma_{1}, \sigma_{2}$ e $\sigma_{3}$ curvas fechadas simples homotopicamente não triviais em $\mathbb{A}$. Então:

(i) Se $\sigma_{1}<\sigma_{2}$ e $\sigma_{2}<\sigma_{3}$, então $\sigma_{1}<\sigma_{3}$.

(ii) Se $\sigma_{1}<\sigma_{2}$, então $h\left(\sigma_{1}\right)<h\left(\sigma_{2}\right)$ para todo homeomorfismo $h: \mathbb{A} \rightarrow \mathbb{A}$ isotópico à identidade.

(iii) $S$ e $\sigma_{3}<\sigma_{1}$ e $\sigma_{3}<\sigma_{2}$, então $\sigma_{3}<\sigma_{1} \vee \sigma_{2}$.

(iv) $\sigma_{1} \vee \sigma_{2} \leq \sigma_{1}$ e $\sigma_{1} \vee \sigma_{2} \leq \sigma_{2}$

(v) $h\left(\sigma_{1} \vee \sigma_{2}\right)=h\left(\sigma_{1}\right) \vee h\left(\sigma_{2}\right)$ para todo homeomorfismo $h: \mathbb{A} \rightarrow \mathbb{A}$, isotópico à identidade.

Finalmente, a operação que associa duas curvas fechadas simples homotopicamente não-triviais $\sigma_{1}, \sigma_{2}$ à curva $\sigma_{1} \vee \sigma_{2}$, é associativa e comutativa. Daí, dado qualquer número finito de curvas fechadas simples homotopicamente não triviais $\sigma_{1}, \ldots, \sigma_{n}$, a curva $\sigma_{1} \vee \ldots \vee \sigma_{n}$ está bem definida. 
Lema 6.1 Seja $h$ um homeomorfismo do anel $\mathbb{A}$, que é isotópico à identidade. Suponhamos existe um inteiro positivo p, e uma curva curva fechada simples homotopicamente não trivial $\sigma$ em $\mathbb{A}$ que é disjunta da sua imagem por $h^{p}$. Então, existe uma curva fechada simples homotopicamente não trivial $\widehat{\sigma}$ em $\mathbb{A}$ que é disjunta da sua imagem por $h$.

Demonstração. Consideremos o inteiro $p$ e a curva $\sigma$ dadas pela hipótese do Lema 6.1. Dado que $\sigma \cap h^{p}(\sigma)=\emptyset$, então ou $\sigma<h^{p}(\sigma)$ ou $h^{p}(\sigma)<\sigma$. Sem perda de generalidade, suponhamos que

$$
\sigma<h^{p}(\sigma) .
$$

Pelo item (ii) da Observação 6.2, temos

$$
h^{-p}(\sigma)<\sigma .
$$

Consideremos assim as curvas fechadas simples homotopicamente não triviais $\tau_{0}, \ldots, \tau_{p-1}$ tais que (ver Figura 6.1)

$$
h^{-p}(\sigma)<\tau_{p-1}<\cdots<\tau_{1}<\tau_{0}=\sigma,
$$

e definimos a curva

$$
\widehat{\sigma}:=\tau_{0} \vee h\left(\tau_{1}\right) \vee \cdots \vee h^{p-1}\left(\tau_{p-1}\right)
$$




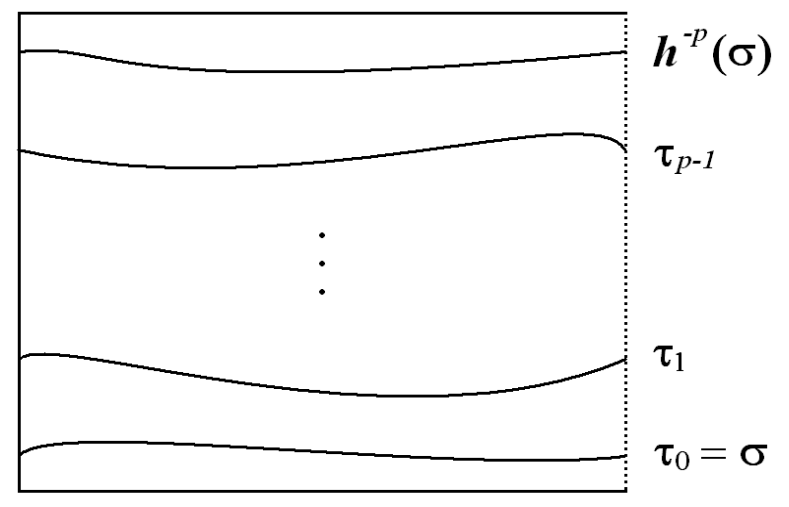

A

Figura 6.1: Curvas homotopicamente não-triviais $\tau_{0}, \ldots, \tau_{p-1}$

Agora, seguindo o mesmo raciocínio feito no início da prova da Proposição 3.1 , obtemos facilmente que

$$
\widehat{\sigma}<h(\widehat{\sigma})
$$

Em outras palavras, conseguimos uma curva fechada simples homotopicamente não trivial $\widehat{\sigma}$ que é disjunta da sua imagem por $h$.

Lema 6.2 Seja h um homeomorfismo do anel $\mathbb{A}$, que é isotópico à identidade. Suponhamos que $h$ não tem pontos fixos. Suponhamos, ainda, que existe um arco essencial simples $\gamma$ em $\mathbb{A}$ que é disjunto de sua imagem por h. Então, para qualquer levantamento $\widetilde{h}$ de $h$, o conjunto de rotação de $\widetilde{h}$ é disjunto de $\mathbb{Z}$.

Demonstração. Consideremos o levantamento $\widetilde{h}$ de $h$ e o levantamento $\Gamma$ de $\gamma$ em $\widetilde{\mathbb{A}}$. Antes de iniciar com a demonstração, note que qualquer 
levantamento de $h$ é da forma $T^{k} \circ \widetilde{h}$ para todo $k \in \mathbb{Z}$. Em particular, pelo Lema 2.2, sabemos que $\operatorname{Rot}\left(T^{k} \circ \widetilde{h}\right)=\operatorname{Rot}(\widetilde{h})+k$. Daí, é suficiente mostrar que 0 não pertence ao conjunto de rotação de $\widetilde{h}$, pois nesse caso, o conjunto de rotação de qualquer levantamento de $h$ será disjunto de $\mathbb{Z}$.

Comecemos notando que pela hipótese sobre o arco $\gamma, \Gamma \cap \widetilde{h}(\Gamma)=\emptyset$. Sem perda de generalidade, e utilizando a mesma notação introduzida na Seção 3.2 , podemos supor que $\Gamma<\widetilde{h}(\Gamma)$, ou seja, $\Gamma \cap R(\widetilde{h}(\Gamma))=\emptyset$. Portanto, $R(\Gamma)$ será um atrator estrito para $\widetilde{h}$. Analogamente, como $\widetilde{h}$ e $T$ comutam, temos que acontece o mesmo para $R\left(T^{2}(\Gamma)\right)$.

Para o próximo passo vamos aplicar um dos resultados da Teoria de Brower sobre homeomorfismos do plano descritos na Seção 2.1. Para isso, lembremos que $\widetilde{h}$ está definido sobre a faixa $\widetilde{\mathbb{A}}=\mathbb{R} \times[0,1]$. Desse modo, faremos uma extensão de $\widetilde{h}$ a todo o plano. Em particular, consideremos a simetria

$$
\begin{aligned}
\chi: \quad \mathbb{R}^{2} & \longrightarrow \mathbb{R}^{2}, \\
(x, y) & \longmapsto(x,-y) .
\end{aligned}
$$

Definimos assim o homeomorfismo $\widetilde{h}^{\prime}: \mathbb{R}^{2} \longrightarrow \mathbb{R}^{2}$ tal que para todo $(x, y) \in \widetilde{\mathbb{A}}$ e $n \in \mathbb{Z}:$

- $\widetilde{h}^{\prime}(x,-y)=\chi(\widetilde{h}(x, y))$;

- $\widetilde{h}^{\prime}(x, y+2 n)=\widetilde{h}^{\prime}(x, y)+(0,2 n)$.

Agora, como o homeomorfismo $h$ não tem pontos fixos, o levantamento $\widetilde{h}$ e o homeomorfismo $\widetilde{h}^{\prime}$ não têm pontos fixos. Similarmente, a extensão do homeomorfismo $\widetilde{h}$ não altera a preservação da orientação no caso do homeomorfismo $\widetilde{h}^{\prime}$. Portanto, pelo Corolário 2.2, afirmamos que qualquer órbita de $\widetilde{h}^{\prime}$ vai para o infinito. 
Consideremos agora qualquer ponto $z \in R(\Gamma)$. Primeiramente, note que a segunda coordenada da órbita de $z$ por $\widetilde{h}$ permanece em $[0,1]$. Além disso, a órbita de $z$ é a mesma por $\widetilde{h}$ e $\widetilde{h}^{\prime}$. Daí, a conclusão do parágrafo anterior permite dizer que o módulo da primeira coordenada ao longo da órbita positiva de $z$ por $\widetilde{h}$, pode tomar valores arbitrariamente grandes. Usando o fato de que $R(\Gamma)$ é um atrator estrito, temos que a primeira coordenada da órbita positiva de $z$ por $\widetilde{h}$ está limitada inferiormente e não está limitada superiormente. Resulta então que existe um inteiro $n(z) \geq 0$ tal que $\widetilde{h}^{n(z)}(z) \in R\left(T^{2}(\Gamma)\right)$. Ainda, como $R\left(T^{2}(\Gamma)\right)$ é um atrator estrito, segue que para todo $n \geq n(z)$ temos que $\widetilde{h}^{n}(z) \in R\left(T^{2}(\Gamma)\right)$.

Analogamente, denotemos por $V_{n(z)}$ uma vizinhança o suficientemente pequena do ponto $z$. Pela continuidade de $\widetilde{h}$ podemos afirmar que para qualquer ponto $z^{\prime}$ que pertence a $V_{n(z)}$ e qualquer $n>n(z)$, o iterado $\widetilde{h}^{n}\left(z^{\prime}\right)$ pertence a $R\left(T^{2}(\Gamma)\right)$.

Seja assim o quadrado $Q=R(\Gamma) \backslash \operatorname{Int}(R(T(\Gamma)))$. Repetindo o argumento anterior em cada ponto de $Q$, segue que

$$
Q \subseteq \cup_{z \in Q} V_{n(z)},
$$

onde para todo ponto $z^{\prime}$ que pertence a $V_{n(z)}$ existe um inteiro $n(z) \geq 1$ (lembre-se que $\Gamma<\widetilde{h}(\Gamma)<T(\Gamma)$ ) tal que para qualquer $n>n(z)$, o iterado $\widetilde{h}^{n}\left(z^{\prime}\right)$ pertence a $R\left(T^{2}(\Gamma)\right)$. Pela compacidade de $Q$, existe um número finito destas vizinhanças que cobrem o quadrado $Q$. Ou seja,

$$
Q \subseteq \cup_{i=1}^{N} V_{n\left(z_{i}\right)} .
$$

Denotando por $n_{0}:=\max _{1 \leq i \leq N}\left\{n_{i}\right\}$, tem-se que para qualquer ponto $z \in Q$ e qualquer $n \geq n_{0} \geq 1$, o iterado $\widetilde{h}^{n}(z)$ pertence a $R\left(T^{2}(\Gamma)\right.$ ). 
Agora, utilizando o conjunto $R(\Gamma) \backslash R(T(\Gamma)) \subset Q$ como domínio fundamental para o recobrimento universal $\widetilde{\mathbb{A}}$, seja então $\omega$ um elemento do conjunto de rotação de $\widetilde{h}$. Assim, existem seqüências $\left(n_{i}\right)_{i=1}^{\infty}$ em $\mathbb{N}$ e $\left(\widetilde{x}_{i}\right)_{i=1}^{\infty}$ em $R(\Gamma) \backslash R(T(\Gamma))$ tais que $n_{i} \rightarrow \infty \mathrm{e}$

$$
\lim _{i \rightarrow \infty} \frac{p_{1}\left(\widetilde{h}^{n} i\left(\widetilde{x}_{i}\right)-\widetilde{x}_{i}\right)}{n_{i}}=\omega .
$$

Nosso objetivo é mostrar que $\omega>0$.

Primeiramente, $n_{i}$ pode ser escrito como $q_{i} n_{0}+r$, onde $0 \leq r<n_{0}-1$, tal que se $i \rightarrow \infty$ então $q_{i} \rightarrow \infty$. Logo,

$$
\frac{p_{1}\left(\widetilde{h}^{n}\left(\widetilde{x}_{i}\right)-\widetilde{x}_{i}\right)}{n_{i}}=\frac{p_{1}\left(\widetilde{h}_{i} n_{0}+r\left(\widetilde{x}_{i}\right)-\widetilde{x}_{i}\right)}{q_{i} n_{0}+r},
$$

além disso,

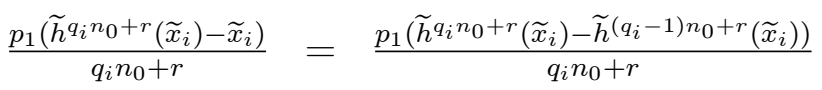

$$
\begin{aligned}
& +\frac{p_{1}\left(\widetilde{h}^{\left(q_{i}-1\right) n_{0}+r}\left(\widetilde{x}_{i}\right)-\widetilde{h}^{\left(q_{i}-2\right) n_{0}+r}\left(\widetilde{x}_{i}\right)\right)}{q_{i} n_{0}+r}+\ldots \\
& +\frac{p_{1}\left(\widetilde{h}^{n_{0}+r}\left(\widetilde{x}_{i}\right)-\widetilde{h}^{r}\left(\widetilde{x}_{i}\right)\right)}{q_{i} n_{0}+r}+\frac{p_{1}\left(\widetilde{h}^{r}\left(\widetilde{x}_{i}\right)-\widetilde{x}_{i}\right)}{q_{i} n_{0}+r} .
\end{aligned}
$$

Para cada $k \in\left\{1, \ldots, q_{i}\right\}$, consideremos os pontos $\widetilde{y}_{k}:=\widetilde{h}^{\left(q_{i}-k\right) n_{0}+r}\left(\widetilde{x}_{i}\right)$. Usando esta notação, a expressão anterior pode-se escrever como

$$
\frac{p_{1}\left(\widetilde{h}_{i} n^{n}+r\left(\widetilde{x}_{i}\right)-\widetilde{x}_{i}\right)}{q_{i} n_{0}+r}=\left(\sum_{k=1}^{q_{i}} \frac{p_{1}\left(\widetilde{h}^{n_{0}}\left(\widetilde{y}_{k}\right)-\widetilde{y}_{k}\right)}{q_{i} n_{0}+r}\right)+\frac{p_{1}\left(\widetilde{h}^{r}\left(\widetilde{x}_{i}\right)-\widetilde{x}_{i}\right)}{q_{i} n_{0}+r} .
$$

Pelo resultado anterior, e sabendo que $\widetilde{h}$ e $T$ comutam, vemos que

$$
p_{1}\left(\widetilde{h}^{n_{0}}\left(\widetilde{y}_{j}\right)-\widetilde{y}_{j}\right) \geq 1 \text {. }
$$

Logo, 


$$
\begin{aligned}
\frac{p_{1}\left(\widetilde{h}^{q_{i} n_{0}+r}\left(\widetilde{x}_{i}\right)-\widetilde{x}_{i}\right)}{q_{i} n_{0}+r} & \geq \frac{q_{i}}{q_{i} n_{0}+r}+\frac{p_{1}\left(\widetilde{h}^{r}\left(\widetilde{x}_{i}\right)-\widetilde{x}_{i}\right)}{q_{i} n_{0}+r} \\
& =\frac{1}{n_{0}+\frac{r}{q_{i}}}+\frac{p_{1}\left(\widetilde{h}^{r}\left(\widetilde{x}_{i}\right)-\widetilde{x}_{i}\right)}{q_{i} n_{0}+r}
\end{aligned}
$$

Tomando o limite quando $i \rightarrow \infty$, obtemos finalmente

$$
\omega \geq \frac{1}{n_{o}}>0
$$

Isto termina a demonstração do Lema 6.2.

\subsection{Prova do Corolário 1.2}

Nesta seção apresentamos a prova do terceiro resultado importante de nosso trabalho. Para isso faremos uso dos Lemas 6.1 e 6.2, e dividiremos a prova em dois casos.

Seja então $h$ um homeomorfismo do anel $\mathbb{A}$, isotópico à identidade, e que não têm pontos periódicos. O Corolário 1.2 apresenta duas propriedades das quais pelo menos uma tem que acontecer.

Suponhamos, como primeiro caso, que a primeira propriedade do Corolário 1.2 acontece, ou seja, existe uma curva fechada simples homotopicamente não trivial $\sigma$ em $\mathbb{A}$, que é disjunta da sua imagem por $h$. Nesse caso, o Corolário1.3 estaria pronto.

Agora, suponhamos que não existe nenhuma curva fechada simples homotopicamente não trivial em $\mathbb{A}$, que é disjunta da sua imagem por $h$. Vejamos como isto implica a segunda propriedade do Corolário 1.2.

Primeiramente, se $h$ não têm pontos periódicos, então para qualquer inteiro $p \neq 0$ vemos que $h^{p}$ não pode ter pontos fixos. 
Apliquemos então o Teorema 6.1 para o homeomorfismo $h^{p}$. Este resultado descreve duas propriedades das quais pelo menos uma têm que acontecer. Suponhamos que acontece a primeira, ou seja, existe um curva fechada simples homotopicamente não trivial em $\mathbb{A}$, disjunta da sua imagem por $h^{p}$. Porém, pelo Lema 6.1, isto implica que existe uma curva fechada simples homotopicamente não trivial em $\mathbb{A}$, disjunta da sua imagem pelo homeomorfismo $h$, o que contradiz nossa hipótese. Portanto, a segunda propriedade do Teorema 6.1 é a que acontece. Esta propriedade diz que existe um arco essencial simples em $\mathbb{A}$, disjunto de sua imagem por $h^{p}$.

Desse modo, aplicando o Lema 6.3 ao homeomorfismo $h^{p}$, temos que o conjunto de rotação para qualquer levantamento $\widetilde{h}^{p}$ de $h^{p}$ é disjunto de $\mathbb{Z}$. Logo, utilizando o Lema 2.2, resulta que o conjunto de rotação de $\widetilde{h}$ é disjunto de $(1 / p) \cdot \mathbb{Z}$. Mas, $p$ é arbitrário. Daí, o conjunto de rotação de $\widetilde{h}$ vai ser disjunto de $\mathbb{Q}$. Dado que o conjunto de rotação de $\widetilde{h}$ é um intervalo compacto, segue que o conjunto de rotação têm que ser só um número irracional, em outras palavras, $h$ é uma pseudo-rotação irracional. Portanto, pelo Teorema 1.1, para todo inteiro positivo $n$, existe um arco essencial simples $\gamma_{n}$ em $\mathbb{A}$, tal que os $\operatorname{arcos} \gamma_{n}, h\left(\gamma_{1}\right), \ldots, h\left(\gamma_{n}\right)$ são dois a dois disjuntos.

Isto termina a prova do Corolário 1.3. 


\section{Referências Bibliográficas}

[1] F. Béguin, S. Crovisier, F. Le Roux and A. Patou, Pseudo-rotations of closed annulus: variation on a theorem of $J$ Kwapisz, Nonlinearity 17 (2004), 1427-1453

[2] F. Béguin, S. Crovisier and F. Le Roux, Pseudo-rotations of open annulus, Bull Braz Math Soc 37 (2006), 275-306

[3] M. Brin and G. Stuck, Introduction to Dynamical Systems, Cambridge University Press, 2002

[4] M. Brown, A New proof of Brouwer's Lemma on Translation Arcs, Houston J. of Math. 10 (1984), 35-41

[5] S. S. Cairns, An elementary proof of the Jordan-Schoenflies theorem, Proc. Am. Math. Soc. 6 (1951), 860-7

[6] G. H. Choe, Computational Ergodic Theory, Springer-Verlag, Berlin, 2005

[7] A. Denjoy, Sur les courbes défines par les équations différentielles à la surface du tore, J. Math. Pure Appl., IX. Ser. 11 (1951), 333-75

[8] M. Flucher, Fixed points of measure preserving torus homeomorphisms, Manuscr. Math. 68 (1990), 271-93 
[9] J. Franks, Generalizations of the Poincaré-Birkhoff theorem, Ann. Math. 128 (1988), 139-51

[10] J. Franks, Recurrence and fixed points of surface homeomorphisms, Ergod. Theory Dynam. Sys. 8 (1988), 99-107

[11] L. Guillou, Théorème de translation plane de Brouwer et généralisations du théorème de Poincaré-Birkhoff, Topology 33 (1994), 331-51

[12] A. Katok and B. Hasselblatt, Introduction to the modern theory of Dynamical Systems, Cambridge University Press, 1999

[13] H. L. Keng, Introduction to Number Theory, Springer-Verlag, Berlin, 1982

[14] J. Kwapisz, A priori degeneracy of one-dimensional rotations sets for periodic point free torus maps, Trans. Am. Math. Soc. 354 (2002), 286595

[15] P. Le Calvez and A. Sauzet, Une démonstration dynamique du théorème de traslation de Brouwer, Exposition Math. 14 (1996), 277-87

[16] P. Le Calvez and J-C Yoccoz, Un théorème d' indice pour les homéomorphismes du plan au voisinage d' un point fixe, Ann. Math. 146 (1997), 241-93

[17] M. Misiurewicz and K. Ziemian, Rotation Sets for maps of Tori, J. London Math. Soc. 40 (1989), 490-506

[18] H. Poincaré, Mémoire sur les courbes définies par une équation différentielle, J. Math Pure. Appl. Série IV 1 167-244 